\title{
Total Synthesis and Structural Elucidation of
}

\section{Azaspiracid-1. Synthesis-Based Analysis of Originally}

\section{Proposed Structures and Indication of Their Non Identity}

\section{to the Natural Product.}

K. C. Nicolaou,* David Y.-K. Chen, Yiwei Li, Noriaki Uesaka, Goran Petrovic, Theocharis V. Koftis, Federico Bernal, Michael O. Frederick, Mugesh Govindasamy, Taotao Ling, Petri M. Pihko, Wenjun Tang, Stepan Vyskocil

Contribution from the Department of Chemistry and The Skaggs Institute for Chemical Biology, The Scripps Research Institute, 10550 North Torrey Pines Road, La Jolla, California 92037, and Department of Chemistry and Biochemistry, University of California, San Diego, 9500 Gilman Drive, La Jolla, California 92093.

Corresponding author e-mail: kcn@scripps.edu

\section{Supporting Information}

\section{Experimental Data for Compounds}

General Procedures. All reactions were carried out under an argon atmosphere with dry solvents under anhydrous conditions, unless otherwise noted. Dry tetrahydrofuran (THF), toluene, benzene, diethyl 
ether $\left(\mathrm{Et}_{2} \mathrm{O}\right)$, ethylene glycol dimethyl ether (DME), and methylene chloride $\left(\mathrm{CH}_{2} \mathrm{Cl}_{2}\right)$ were obtained by passing commercially available pre-dried, oxygen-free formulations through activated alumina columns. Yields refer to chromatographically and spectroscopically ( ${ }^{1} \mathrm{H}$ NMR) homogeneous materials, unless otherwise stated. Reagents were purchased at the highest commercial quality and used without further purification, unless otherwise stated. Reactions were monitored by thin-layer chromatography (TLC) carried out on $0.25 \mathrm{~mm}$ E. Merck silica gel plates (60F-254) using UV light as visualizing agent and an ethanolic solution of phosphomolybdic acid and cerium sulfate, and heat as developing agents. E. Merck silica gel (60, particle size $0.040-0.063 \mathrm{~mm})$ was used for flash column chromatography. Preparative thin-layer chromatography (PTLC) separations were carried out on 0.25 or $0.50 \mathrm{~mm}$ E. Merck silica gel plates (60F-254). NMR spectra were recorded on Bruker DRX-600, DRX-500, AMX-500 or AMX-400 instruments and calibrated using residual undeuterated solvent as an internal reference. The following abbreviations were used to explain the multiplicities: $\mathrm{s}=$ singlet, $\mathrm{d}=$ doublet, $\mathrm{t}=$ triplet, $\mathrm{q}=$ quartet, $\mathrm{m}=$ multiplet, quin $=$ quintuplet, sext $=$ sextet, sep $=$ septet, $b r=$ broad. $I R$ spectra were recorded on a Perkin-Elmer 1600 series FT-IR spectrometer. Electrospray ionization (ESI) mass spectrometry (MS) experiments were performed on an API 100 Perkin Elmer SCIEX single quadrupole mass spectrometer at $4000 \mathrm{~V}$ emitter voltage. High-resolution mass spectra (HRMS) were recorded on a VG ZAB-ZSE mass spectrometer using MALDI (matrix-assisted laser-desorption ionization) or ESI (electrospray ionization).

Primary Alcohol 14. To a solution of silyl ether $13(200 \mathrm{mg}, 0.31 \mathrm{mmol}, 1.0$ equiv) in THF $(10 \mathrm{~mL})$ at $25{ }^{\circ} \mathrm{C}$ was added TBAF $(1.5 \mathrm{~mL}, 1.0 \mathrm{M}$ in THF, $1.5 \mathrm{mmol}, 5.0$ equiv). The reaction mixture was

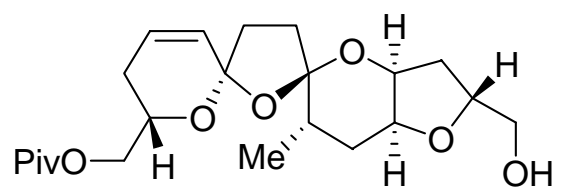

14 allowed to stir for $2 \mathrm{~h}$ at $25{ }^{\circ} \mathrm{C}$ and then quenched with saturated aqueous $\mathrm{NH}_{4} \mathrm{Cl}(15 \mathrm{~mL})$. The biphasic mixture was extracted with EtOAc $(3 \times 10 \mathrm{~mL})$ and the combined organic extracts were dried $\left(\mathrm{Na}_{2} \mathrm{SO}_{4}\right)$, concentrated, and then purified by flash column chromatography (silica gel, EtOAc:hexanes 3:1) to afford primary alcohol $14(112 \mathrm{mg}, 0.27 \mathrm{mmol}, 88 \%)$ as a colorless oil. 14: $R_{\mathrm{f}}=0.19$ (silica gel, EtOAc:hexanes 3:1); $[\alpha]_{\mathrm{D}}^{25}=-31.2\left(\mathrm{CHCl}_{3}, c=1.10\right) ;$ IR (film) $v_{\max }=3482,2957,2928,1729,1455$, 
$1159 \mathrm{~cm}^{-1} ;{ }^{1} \mathrm{H}$ NMR $\left(400 \mathrm{MHz}, \mathrm{CDCl}_{3}\right): \delta=5.97$ (ddd, $\left.J=10.0,5.5,2.5 \mathrm{~Hz}, 1 \mathrm{H}\right), 5.70(\mathrm{ddd}, J=10.0$, 3.0, $1.0 \mathrm{~Hz}, 1 \mathrm{H}), 4.48$ (dddd, $J=11.5,9.0,6.5,3.0 \mathrm{~Hz}, 1 \mathrm{H}), 4.21$ (ddd, $J=15.0,10.0,5.0 \mathrm{~Hz}, 1 \mathrm{H})$, $4.17(\mathrm{dd}, J=3.5,2.5 \mathrm{~Hz}, 1 \mathrm{H}), 4.07(\mathrm{~d}, J=12.0 \mathrm{~Hz}, 1 \mathrm{H}), 4.06(\mathrm{~d}, J=12.0 \mathrm{~Hz}, 1 \mathrm{H}), 3.85(\mathrm{~d}, J=2.5 \mathrm{~Hz}$, $1 \mathrm{H}), 3.73$ (ddd, $J=11.5,3.0,1.0 \mathrm{~Hz}, 1 \mathrm{H}), 3.49-3.46(\mathrm{~m}, 1 \mathrm{H}), 2.21(\mathrm{ddd}, J=13.0,7.0,4.0 \mathrm{~Hz}, 1 \mathrm{H})$, 2.11-2.02 (m, 4 H), 2.00-1.86 (m, 6 H), 1.46 (ddd, $J=16.5,4.0,3.0 \mathrm{~Hz}, 1 \mathrm{H}), 1.18(\mathrm{~s}, 9 \mathrm{H}), 0.96(\mathrm{~d}, J=$ $7.0 \mathrm{~Hz}, 3 \mathrm{H}) ;{ }^{13} \mathrm{C} \mathrm{NMR}\left(150 \mathrm{MHz}, \mathrm{CDCl}_{3}\right): \delta=178.4,129.2,127.4,111.4,103.9,78.9,76.2,75.8,66.5$, 66.4, 64.7, 38.7, 35.7, 35.6, 33.8, 30.8, 27.1, 26.5, 23.3, 15.9; HR-MS [Matrix-assisted laser desorption / ionization (MALDI)]: calcd for $\mathrm{C}_{22} \mathrm{H}_{34} \mathrm{O}_{7} \mathrm{Na}^{+}\left[\mathrm{M}+\mathrm{Na}^{+}\right]$: 433.2197, found 433.2197.

Aldehyde 10. To a solution of oxalyl chloride $\left(0.12 \mathrm{~mL}, 1.35 \mathrm{mmol}, 5.0\right.$ equiv) in $\mathrm{CH}_{2} \mathrm{Cl}_{2}(6 \mathrm{~mL})$ at -78 ${ }^{\circ} \mathrm{C}$ was added DMSO (0.19 mmol, $3.0 \mathrm{mmol}, 11.0$ equiv). The solution was allowed to stir for $20 \mathrm{~min}$ at

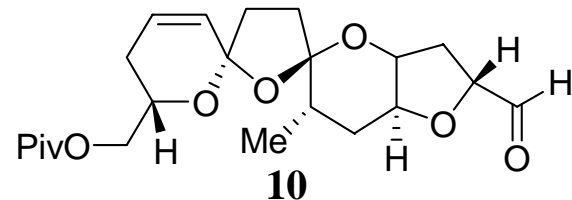
$-78{ }^{\circ} \mathrm{C}$ and then a solution of primary alcohol $14(112 \mathrm{mg}, 0.27$ mmol, 1.0 equiv) in $\mathrm{CH}_{2} \mathrm{Cl}_{2}(5 \mathrm{~mL})$ was added. The resulting mixture was allowed to stir at $-78{ }^{\circ} \mathrm{C}$ for $1 \mathrm{~h}$ at which time $\mathrm{Et}_{3} \mathrm{~N}(0.86 \mathrm{~mL}$, $6.0 \mathrm{mmol}, 22.0$ equiv) was added. The reaction mixture was allowed to warm to $0{ }^{\circ} \mathrm{C}$ over $1 \mathrm{~h}$, and was then quenched with saturated aqueous $\mathrm{NaCl}(15 \mathrm{~mL})$. The biphasic mixture was extracted with $\mathrm{CH}_{2} \mathrm{Cl}_{2}$ $(3 \times 10 \mathrm{~mL})$ and the combined organic extracts were dried $\left(\mathrm{Na}_{2} \mathrm{SO}_{4}\right)$, concentrated, and then purified by flash column chromatography (silica gel, EtOAc:hexanes 2:1) to give aldehyde 10 (105 mg, 0.25 mmol, 94\%) as a colorless oil. 10: $R_{\mathrm{f}}=0.39$ (silica gel, EtOAc:hexanes $\left.3: 1\right) ;[\alpha]_{\mathrm{D}}{ }^{25}=-27.9\left(\mathrm{CHCl}_{3}, c=0.90\right)$; IR (film) $v_{\max }=3562,1731,1715,1455,1286,1159 \mathrm{~cm}^{-1} ;{ }^{1} \mathrm{H}$ NMR $\left(400 \mathrm{MHz}, \mathrm{CDCl}_{3}\right): \delta=9.70(\mathrm{~d}, J=$ $1.6 \mathrm{~Hz}, 1 \mathrm{H}), 5.99$ (ddd, $J=10.0,5.0,2.0 \mathrm{~Hz}, 1 \mathrm{H}), 5.71$ (ddd, $J=10.0,3.0,2.0 \mathrm{~Hz}, 1 \mathrm{H}), 4.69$ (ddd, $J=$ 9.2, 8.0, 1.6 Hz, 1 H), 4.25-4.22 (m, $1 \mathrm{H}), 4.21-4.19(\mathrm{~m}, 1 \mathrm{H}), 4.07$ (d, J = $12.0 \mathrm{~Hz}, 1 \mathrm{H}), 4.06(\mathrm{~d}, J=$ $12.0 \mathrm{~Hz}, 1 \mathrm{H}), 3.89(\mathrm{dd}, J=4.8,2.0 \mathrm{~Hz}, 1 \mathrm{H}), 2.35(\mathrm{dd}, J=13.5,7.5 \mathrm{~Hz}, 1 \mathrm{H}), 2.26-2.22(\mathrm{~m}, 1 \mathrm{H})$, $2.11-1.91(\mathrm{~m}, 8 \mathrm{H}), 1.50(\mathrm{ddd}, J=15.5,12.4,3.2 \mathrm{~Hz}, 1 \mathrm{H}), 1.19(\mathrm{~s}, 9 \mathrm{H}), 0.98(\mathrm{~d}, J=7.0 \mathrm{~Hz}, 3 \mathrm{H}) ;{ }^{13} \mathrm{C}$ $\operatorname{NMR}\left(150 \mathrm{MHz}, \mathrm{CDCl}_{3}\right): \delta=202.3,178.4,129.1,127.6,111.5,104.0,82.2,77.2,75.1,66.6,66.5$, 35.7, 35.6, 33.3, 30.6, 27.1, 26.4, 23.4, 19.3, 15.8; HR-MS [Matrix-assisted laser desorption / ionization (MALDI)]: calcd for $\mathrm{C}_{22} \mathrm{H}_{32} \mathrm{O}_{7} \mathrm{Na}^{+}\left[\mathrm{M}+\mathrm{Na}^{+}\right]$: 431.2040, found 431.2029. 
PFP Ester 11. To a solution of aldehyde 10 (105 mg, $0.25 \mathrm{mmol}, 1.0$ equiv) dissolved in $t-\mathrm{BuOH}: \mathrm{H}_{2} \mathrm{O}$ (4:1, $5 \mathrm{~mL}$ ) at $25^{\circ} \mathrm{C}$, was added $\mathrm{NaClO}_{2}\left(135 \mathrm{mg}, 1.5 \mathrm{mmol}, 6.0\right.$ equiv), $\mathrm{NaH}_{2} \mathrm{PO}_{4}$ (234 mg, $1.5 \mathrm{mmol}$,

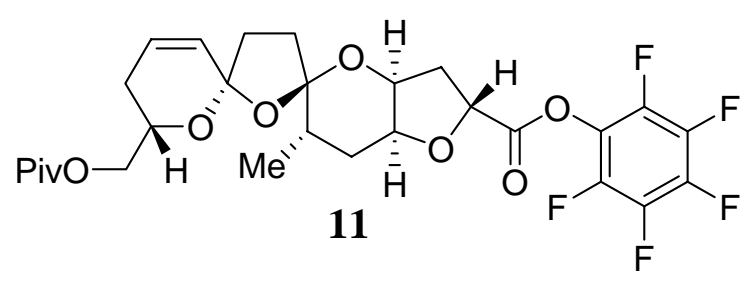
6.0 equiv), and 2-methyl-2-butene $(0.27 \mathrm{~mL}, 2.5 \mathrm{mmol}$, 10.0 equiv). The reaction mixture was allowed to stir at 25 ${ }^{\circ} \mathrm{C}$ for $1.5 \mathrm{~h}$, then quenched with a $\mathrm{pH} 7$ buffered saline solution $(5 \mathrm{~mL})$. The biphasic mixture was then extracted with EtOAc $(3 \times 4 \mathrm{~mL})$ and the combined organic extracts were dried $\left(\mathrm{Na}_{2} \mathrm{SO}_{4}\right)$, and concentrated, and the obtained crude carboxylic acid $\mathbf{1 5}$ was used in the next step without further purification. To a solution of crude carboxylic acid $15\left(107 \mathrm{mg}, 0.25 \mathrm{mmol}, 1.0\right.$ equiv) in $\mathrm{CH}_{2} \mathrm{Cl}_{2}(3 \mathrm{~mL})$ at $25{ }^{\circ} \mathrm{C}$ was added DCC (103 mg, $0.50 \mathrm{mmol}, 2.0$ equiv) followed by pentafluorophenol (69 mg, $0.38 \mathrm{mmol}, 1.5$ equiv). The reaction mixture was allowed to stir at $25{ }^{\circ} \mathrm{C}$ for $2.5 \mathrm{~h}$, and was then concentrated and purified by flash column chromatography (silica gel, $\mathrm{Et}_{2} \mathrm{O}$ :hexanes 3:7) to afford PFP ester 11 (115 mg, $0.195 \mathrm{mmol}, 78 \%$ over two steps) as a colorless oil. 11: $R_{\mathrm{f}}=0.31$ (silica gel, EtOAc:hexanes 1:4); $[\alpha]_{\mathrm{D}}{ }^{25}$ $=-34.2\left(\mathrm{CHCl}_{3}, c=0.50\right)$; IR (film) $v_{\max }=3649,2933,1793,1734,1521,1159 \mathrm{~cm}^{-1} ;{ }^{1} \mathrm{H}$ NMR $(600$ $\left.\mathrm{MHz}, \mathrm{CDCl}_{3}\right): \delta=6.00(\mathrm{ddd}, J=10.0,6.0,2.0 \mathrm{~Hz}, 1 \mathrm{H}), 5.71(\mathrm{~d}, J=10.0 \mathrm{~Hz}, 1 \mathrm{H}), 5.15(\mathrm{t}, J=8.0,1$ H), 4.27 (br s, 1 H), 4.22 (ddd, J = 14.5, 9.0, 4.5 Hz, 1 H), 4.10 (br s, 1 H), 4.08-4.06 (m, 2 H), 2.66 (dd, $J=13.0,7.5 \mathrm{~Hz}, 1 \mathrm{H}), 2.32$ (ddd, $J=9.0,4.5,4.0 \mathrm{~Hz}, 1 \mathrm{H}), 2.28-2.22(\mathrm{~m}, 2 \mathrm{H}), 2.13-2.09$ (m, $1 \mathrm{H})$, 2.07-1.94 (m, $4 \mathrm{H}), 1.70$ (dt, $J=9.5,4.0 \mathrm{~Hz}, 1 \mathrm{H}), 1.53-1.50$ (m, $1 \mathrm{H}), 1.29$ (s, $9 \mathrm{H}), 0.99$ (d, J = 7.0 Hz, $3 \mathrm{H}) ;{ }^{13} \mathrm{C}$ NMR $\left(150 \mathrm{MHz}, \mathrm{CDCl}_{3}\right): \delta=178.5,169.8,156.2,153.1,147.5,138.6,129.0,127.7,111.6$, 104.1, 77.7, 77.5, 75.0, 66.6, 66.5, 39.0, 38.8, 35.6, 33.0, 30.5, 27.1, 26.4, 23.4, 15.8; HR-MS [Matrixassisted laser desorption / ionization (MALDI)]: calcd for $\mathrm{C}_{28} \mathrm{H}_{31} \mathrm{~F}_{5} \mathrm{O}_{8} \mathrm{Na}^{+}\left[\mathrm{M}+\mathrm{Na}^{+}\right]$: 613.1831, found 613.1844 .

Coupling Product 16. To a solution of dithiane 8 ( $734 \mathrm{mg}, 1.76 \mathrm{mmol}, 9.0$ equiv) in THF (5 mL) at 25 ${ }^{\circ} \mathrm{C}$ was added a mixture of $n-\mathrm{BuLi}-n-\mathrm{Bu}_{2} \mathrm{Mg}(1.06 \mathrm{~mL}, 1.1 \mathrm{M}$ in hexanes, $1.17 \mathrm{mmol}, 6.0$ equiv). The resulting solution was allowed to stir for $1.5 \mathrm{~h}$ at $25^{\circ} \mathrm{C}$, and was then cooled to $-90{ }^{\circ} \mathrm{C}$. To the dithiane 
solution was added a solution of PFP ester 11 (115 mg, $0.195 \mathrm{mmol}, 1.0$ equiv) in THF (2 mL), and the reaction mixture was allowed to stir at $-90{ }^{\circ} \mathrm{C}$ for $15 \mathrm{~min}$ and then quenched with saturated aqueous

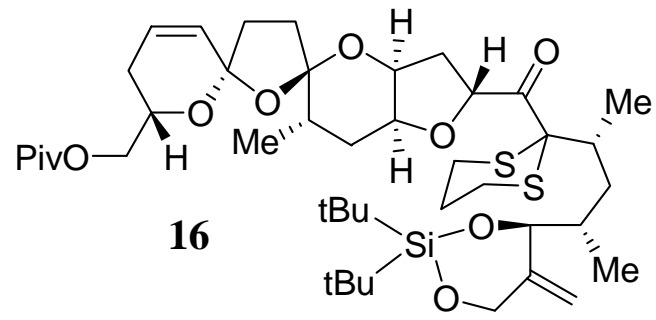
$\mathrm{NaHCO}_{3}(5 \mathrm{~mL})$. The resulting biphasic mixture was extracted with EtOAc $(3 \times 5 \mathrm{~mL})$ and the combined organic extracts were dried $\left(\mathrm{Na}_{2} \mathrm{SO}_{4}\right)$, concentrated, and then purified by flash column chromatography (silica gel, EtOAc:hexanes $1: 9 \rightarrow 1: 1$ ) to provide coupling product $16(101 \mathrm{mg}, 0.12 \mathrm{mmol}, 63 \%)$ as a colorless oil. 16: $R_{\mathrm{f}}=0.59$ (silica gel, EtOAc:hexanes 1:2); $[\alpha]_{\mathrm{D}}^{25}=-24.8\left(\mathrm{CHCl}_{3}, c=1.50\right)$; IR (film) $v_{\max }=3648,2930,1734,1715,1516$, $1082 \mathrm{~cm}^{-1} ;{ }^{1} \mathrm{H}$ NMR $\left(600 \mathrm{MHz}, \mathrm{CDCl}_{3}\right): \delta=5.96(\mathrm{ddd}, J=10.0,5.5,2.0 \mathrm{~Hz}, 1 \mathrm{H}), 5.69(\mathrm{dd}, J=10.0$, $1.5 \mathrm{~Hz}, 1 \mathrm{H}), 5.36(\mathrm{dd}, J=9.0,7.0 \mathrm{~Hz}, 1 \mathrm{H}), 5.10(\mathrm{~s}, 1 \mathrm{H}), 4.95(\mathrm{~s}, 1 \mathrm{H}), 4.54(\mathrm{~d}, J=11.6 \mathrm{~Hz}, 1 \mathrm{H})$, 4.26-4.16 (m, $5 \mathrm{H}), 4.07$ (d, $J=12.0 \mathrm{~Hz}, 1 \mathrm{H}), 4.06$ (d, $J=12.0 \mathrm{~Hz}, 1 \mathrm{H}), 2.95-2.82$ (m, $2 \mathrm{H}), 2.60$ (ddd, $J=9.0,5.0,4.0 \mathrm{~Hz}, 2 \mathrm{H}), 2.31-2.27(\mathrm{~m}, 2 \mathrm{H}), 2.23-2.16(\mathrm{~m}, 3 \mathrm{H}), 2.17-2.07(\mathrm{~m}, 3 \mathrm{H}), 2.03-1.95$ (m, 6 H), 1.91-1.88 (m, $1 \mathrm{H}), 1.72-1.69(\mathrm{~m}, 1 \mathrm{H}), 1.48-1.42(\mathrm{~m}, 1 \mathrm{H}), 1.19(\mathrm{~s}, 9 \mathrm{H}), 1.12(\mathrm{~d}, J=6.6 \mathrm{~Hz}, 3 \mathrm{H})$, 1.04 (s, $9 \mathrm{H}), 0.98$ (s, $9 \mathrm{H}), 0.94$ (d, J = 7.0 Hz, $3 \mathrm{H}), 0.88(\mathrm{~d}, J=7.0 \mathrm{~Hz}, 3 \mathrm{H}) ;{ }^{13} \mathrm{C} \mathrm{NMR}(150 \mathrm{MHz}$ $\left.\mathrm{CDCl}_{3}\right): \delta=178.5,145.8,129.3,127.4,114.3,111.6,103.9,80.6,77.6,76.2,75.9,69.4,67.7,66.6$, $66.5,40.4,38.7,38.3,36.3,35.7,35.1,33.2,30.5,29.6,27.3,27.1,27.0,26.4,24.6,23.2,21.5,21.0$, 17.2, 16.7, 15.9; HR-MS [Matrix-assisted laser desorption / ionization (MALDI)]: calcd for $\mathrm{C}_{43} \mathrm{H}_{70} \mathrm{O}_{9} \mathrm{~S}_{2} \mathrm{SiNa}^{+}\left[\mathrm{M}+\mathrm{Na}^{+}\right]:$845.4122, found 845.4132.

Reduced Product 17. To a solution of coupling product 16 (101 mg, $0.12 \mathrm{mmol}, 1.0$ equiv) in $\mathrm{CH}_{2} \mathrm{Cl}_{2}$ (3 $\mathrm{mL})$ at $-90{ }^{\circ} \mathrm{C}$ was added DIBAL-H (1.2 mL, $1.0 \mathrm{M}$ in $\mathrm{CH}_{2} \mathrm{Cl}_{2}, 1.2 \mathrm{mmol}, 10.0$ equiv). The reaction

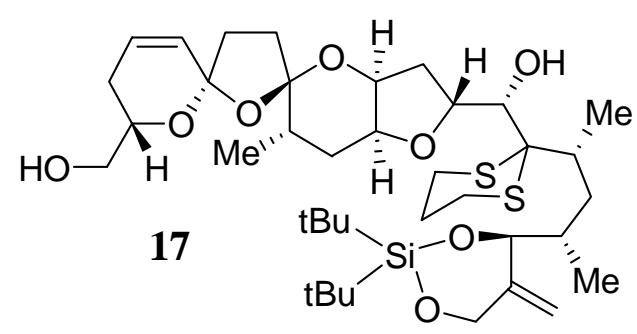
mixture was maintained at $-90{ }^{\circ} \mathrm{C}$ for $1.5 \mathrm{~h}$ at which time it was quenched with $\mathrm{MeOH}(0.3 \mathrm{~mL})$, saturated aqueous sodium and potassium tartrate $(5 \mathrm{~mL})$ and $\mathrm{H}_{2} \mathrm{O}(2 \mathrm{~mL})$ and allowed to warm to $25{ }^{\circ} \mathrm{C}$ over $2 \mathrm{~h}$. The resulting biphasic mixture was then extracted with $\mathrm{CH}_{2} \mathrm{Cl}_{2}(3 \times 5 \mathrm{~mL})$ and the combined organic extracts were dried $\left(\mathrm{Na}_{2} \mathrm{SO}_{4}\right)$, concentrated, and then purified by flash column chromatography (silica 
gel, EtOAc:hexanes 2:1) to afford a single isomer of reduced product 17 (49 $\mathrm{mg}, 0.066 \mathrm{mmol}, 55 \%)$ as a colorless oil. 17: $R_{\mathrm{f}}=0.40$ (silica gel, EtOAc:hexanes 1:1); $[\alpha]_{\mathrm{D}}{ }^{25}=-38.5\left(\mathrm{CHCl}_{3}, c=1.50\right)$; IR (film) $v_{\max }=3510,2929,1458,1384,1260,1081 \mathrm{~cm}^{-1} ;{ }^{1} \mathrm{H} \mathrm{NMR}\left(600 \mathrm{MHz}, \mathrm{CDCl}_{3}\right): \delta=6.00(\mathrm{ddd}, J=10.0$, 6.0, 2.0 Hz, $1 \mathrm{H}), 5.71(\mathrm{dd}, J=10.0,2.0 \mathrm{~Hz}, 1 \mathrm{H}), 5.08(\mathrm{~s}, 1 \mathrm{H}), 4.94(\mathrm{~s}, 1 \mathrm{H}), 4.88(\mathrm{t}, J=8.3 \mathrm{~Hz}, 1 \mathrm{H})$, $4.54(\mathrm{~d}, J=12.0 \mathrm{~Hz}, 1 \mathrm{H}), 4.29(\mathrm{~d}, J=6.0 \mathrm{~Hz}, 1 \mathrm{H}), 4.18(\mathrm{~d}, J=12.0 \mathrm{~Hz}, 1 \mathrm{H}), 4.17-4.14(\mathrm{~m}, 1 \mathrm{H})$, 4.13-4.10 (m, $1 \mathrm{H}), 3.90$ (br s, $1 \mathrm{H}), 3.69$ (d, $J=10.0 \mathrm{~Hz}, 1 \mathrm{H}), 3.64$ (d, J=9.0 Hz, $1 \mathrm{H}), 3.57$ (dd, $J=$ 11.0, 6.0 Hz, 1 H), 3.17-3.12 (m, $1 \mathrm{H}), 3.07$ (d, $J=9.0 \mathrm{~Hz}, 1 \mathrm{H}), 2.83$ (ddd, $J=9.0,4.0,3.0 \mathrm{~Hz}, 1 \mathrm{H})$, 2.72-2.69 (m, $1 \mathrm{H}), 2.58(\mathrm{dt}, J=13.5,4.5 \mathrm{~Hz}, 1 \mathrm{H}), 2.32(\mathrm{dd}, J=14.0,3.0 \mathrm{~Hz}, 1 \mathrm{H}), 2.18-2.09$ (m, $7 \mathrm{H})$, 2.01-1.95 (m, $4 \mathrm{H}), 1.88-1.80(\mathrm{~m}, 4 \mathrm{H}), 1.25-1.16(\mathrm{~m}, 5 \mathrm{H}), 1.04(\mathrm{~s}, 9 \mathrm{H}), 0.98(\mathrm{~s}, 9 \mathrm{H}), 0.93(\mathrm{~d}, J=7.0$ $\mathrm{Hz}, 3 \mathrm{H}), 0.89(\mathrm{~d}, J=7.0 \mathrm{~Hz}, 3 \mathrm{H}) ;{ }^{13} \mathrm{C} \mathrm{NMR}\left(150 \mathrm{MHz}, \mathrm{CDCl}_{3}\right): \delta=146.5,129.1,127.9,113.7,111.4$, $104.0,81.0,76.5,75.9,68.9,68.2,68.1,65.3,62.7,40.2,39.3,39.2,36.0,35.7,34.1,30.9,29.6,27.3$, 27.1, 26.9, 26.1, 25.4, 21.4, 21.1, 17.4, 17.3, 15.7; HR-MS [Matrix-assisted laser desorption / ionization (MALDI)]: calcd for $\mathrm{C}_{38} \mathrm{H}_{64} \mathrm{O}_{8} \mathrm{~S}_{2} \mathrm{SiNa}^{+}\left[\mathrm{M}+\mathrm{Na}^{+}\right]$: 763.3704, found 763.3722.

ABCDE Ring System 21. Reduced product 17 (25 mg, $0.036 \mathrm{mmol}, 1.0$ equiv) was dissolved in THF (5 $\mathrm{mL})$ and cooled to $0{ }^{\circ} \mathrm{C}$. TBAF $(0.14 \mathrm{~mL}, 1.0 \mathrm{M}$ in THF, $0.14 \mathrm{mmol}, 4.0$ equiv) was added and the

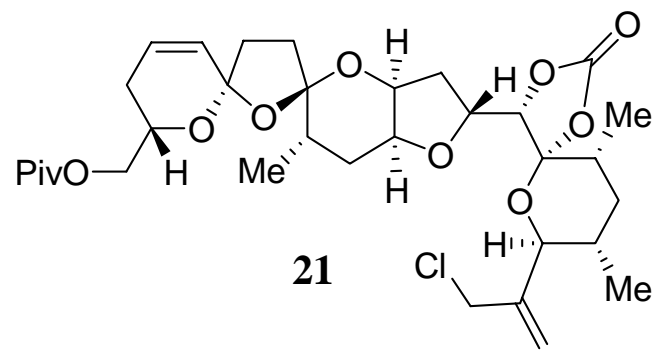
mixture was stirred for $15 \mathrm{~h}$ at $25^{\circ} \mathrm{C}$. The reaction mixture was then quenched with saturated aqueous $\mathrm{NH}_{4} \mathrm{Cl}(5 \mathrm{~mL})$ and then extracted with EtOAc $(3 \times 10 \mathrm{~mL})$. The combined organic extracts were dried $\left(\mathrm{Na}_{2} \mathrm{SO}_{4}\right)$ and concentrated, and the obtained crude pentacycle 20 was carried on to the next step without further purification. The newly prepared pentacycle 20 (20 mg, $0.036 \mathrm{mmol}, 1.0$ equiv) was dissolved in a mixture of $\mathrm{MeCN}: \mathrm{pH} 7$ buffered saline solution $(4: 1,5 \mathrm{~mL})$ and cooled to $0{ }^{\circ} \mathrm{C}$. $\mathrm{PhI}\left(\mathrm{OCOCF}_{3}\right)_{2}(0.11$ $\mathrm{mL}, 0.5 \mathrm{M}$ in $\mathrm{MeCN}, 0.055 \mathrm{mmol}, 1.5$ equiv) was added and the reaction mixture was stirred at $0{ }^{\circ} \mathrm{C}$ for $15 \mathrm{~min}$ and then quenched with saturated aqueous $\mathrm{Na}_{2} \mathrm{~S}_{2} \mathrm{O}_{3}(5 \mathrm{~mL})$. The aqueous layer was extrated with EtOAc $(3 \times 10 \mathrm{~mL})$ and the combined organic layers were washed with water $(20 \mathrm{~mL})$ and brine $(30$ $\mathrm{mL}$ ), dried over $\mathrm{Na}_{2} \mathrm{SO}_{4}$, and concentrated. The crude product was dissolved in $\mathrm{CH}_{2} \mathrm{Cl}_{2}(5 \mathrm{~mL})$ and 
cooled to $-78^{\circ} \mathrm{C}$. Pyridine $(0.18 \mathrm{~mL}, 2.2 \mathrm{mmol}, 15$ equiv) was added followed by triphosgene (32 $\mathrm{mg}$, $0.11 \mathrm{mmol}, 2.0$ equiv). The reaction mixture was allowed to warm up to $0{ }^{\circ} \mathrm{C}$ and stirred for $1 \mathrm{~h}$. The reaction mixture was quenched with saturated aqueous $\mathrm{NaHCO}_{3}(5 \mathrm{~mL})$ and extracted with EtOAc $(3 \times$ $10 \mathrm{~mL})$. The combined organic extracts were washed with brine $(10 \mathrm{~mL})$, dried over $\mathrm{Na}_{2} \mathrm{SO}_{4}$, filtered, concentrated, and then purified by flash column chromatography (silica gel, hexanes:EtOAc, 2:1) to afford allylic chloride 21 ( $8.3 \mathrm{mg}, 0.013 \mathrm{mmol}, 36 \%$ over three steps) as a colorless, viscous oil. 21: $R_{\mathrm{f}}=$ 0.30 (silica gel, EtOAc:hexanes 1:1); $[\alpha]_{\mathrm{D}}=+9.8\left(c=2.00, \mathrm{CHCl}_{3}\right) ; \mathrm{IR}\left(\right.$ film) $v_{\max }=3436,2930,1725$, $1725,1460,1402,1350,1155,1025,979,879,585 \mathrm{~cm}^{-1} ;{ }^{1} \mathrm{H}$ NMR $\left(500 \mathrm{MHz}, \mathrm{CDCl}_{3}\right): \delta=5.98(\mathrm{ddd}, J$ $=9.8,5.6,2.1 \mathrm{~Hz}, 1 \mathrm{H}), 5.70(\mathrm{ddd}, J=9.8,2.3,1.0 \mathrm{~Hz}, 1 \mathrm{H}), 5.42(\mathrm{~s}, 1 \mathrm{H}), 5.22(\mathrm{~s}, 1 \mathrm{H}), 4.66(\mathrm{dt}, J=$ 10.1, 6.1 Hz, 1 H), $4.31(\mathrm{~d}, J=6.1 \mathrm{~Hz}, 1 \mathrm{H}), 4.24-4.20(\mathrm{~m}, 1 \mathrm{H}), 4.15(\mathrm{~m}, 1 \mathrm{H}), 4.14-4.09(\mathrm{~m}, 3 \mathrm{H})$, 4.08-4.05 (m, 2 H), 3.89 (m, $1 \mathrm{H}), 2.23-2.20$ (m, $2 \mathrm{H}), 2.16-2.13$ (m, $1 \mathrm{H}), 2.11-2.07$ (m, 2 H), 2.04$1.94(\mathrm{~m}, 6 \mathrm{H}), 1.87-1.77$ (m, $2 \mathrm{H}), 1.46-1.37$ (m, $2 \mathrm{H}), 1.19$ (s, $9 \mathrm{H}), 1.02$ (d, J = 6.6 Hz, $3 \mathrm{H}), 0.96$ (d, J $=6.6 \mathrm{~Hz}, 3 \mathrm{H}), 0.86(\mathrm{~d}, J=6.6 \mathrm{~Hz}, 3 \mathrm{H}) ;{ }^{13} \mathrm{C} \mathrm{NMR}\left(125 \mathrm{MHz}, \mathrm{CDCl}_{3}\right): \delta=178.9,146.4,129.6,128.1$, $118.1,112.0,104.5,100.4,98.6,81.5,78.2,75.9,75.6,73.7,67.0,66.9,63.4,38.7,36.7,36.1,34.3$, 33.7, 31.1, 27.6, 27.0, 24.6, 24.2, 23.8, 18.0, 16.5, 16.3; HRMS (MALDI-FTMS) for $\mathrm{C}_{33} \mathrm{H}_{47} \mathrm{ClO}_{10} \mathrm{Na}^{+}$ $\left[\mathrm{M}+\mathrm{Na}^{+}\right]$calcd 661.2750 , found 661.2749 .

Primary alcohol 23. To a solution of silyl ether $22(218 \mathrm{mg}, 0.31 \mathrm{mmol}, 1.0$ equiv) in THF (10 mL) at $25{ }^{\circ} \mathrm{C}$ was added TBAF $(1.5 \mathrm{~mL}, 1.0 \mathrm{M}$ in THF, $1.5 \mathrm{mmol}, 5.0$ equiv). The reaction mixture was

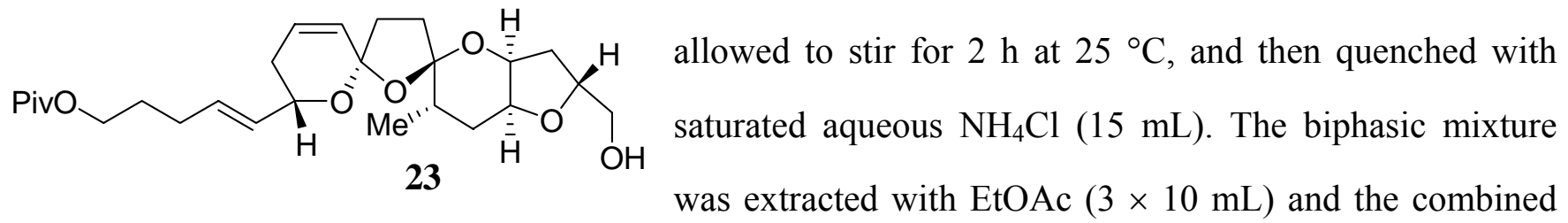
organic extracts were dried $\left(\mathrm{Na}_{2} \mathrm{SO}_{4}\right)$, concentrated, and then purified by flash column chromatography (silica gel, EtOAc:hexanes 5:1) to afford primary alcohol 23 (134 mg, $0.29 \mathrm{mmol}, 93 \%)$ as a colorless oil. 23: $R_{\mathrm{f}}=0.30$ (silica gel, EtOAc); $[\alpha]_{\mathrm{D}}=-59.2\left(c=1.60, \mathrm{CHCl}_{3}\right)$; IR (film) $v_{\max }=3440,2960,2924$, 1728, 1455, 1321, 1281, 1230, $1094 \mathrm{~cm}^{-1} ;{ }^{1} \mathrm{H}$ NMR (600 MHz, $\left.\mathrm{CDCl}_{3}\right): \delta=5.97-5.93(\mathrm{~m}, 1 \mathrm{H}), 5.69-$ $5.63(\mathrm{~m}, 2 \mathrm{H}), 5.48$ (dd, $J=12.5,6.0 \mathrm{~Hz}, 1 \mathrm{H}), 4.45$ (br s, $1 \mathrm{H}), 4.40-4.37$ (m, $1 \mathrm{H}), 4.15$ (br s, $1 \mathrm{H})$, 
$4.01(\mathrm{t}, J=6.0 \mathrm{~Hz}, 2 \mathrm{H}), 3.82(\mathrm{~s}, 1 \mathrm{H}), 3.69(\mathrm{dd}, J=12.0,2.4 \mathrm{~Hz}, 1 \mathrm{H}), 3.44(\mathrm{dd}, J=12.0,5.0 \mathrm{~Hz}, 1 \mathrm{H})$, 2.83-2.80 (m, $1 \mathrm{H}), 2.18-2.14(\mathrm{~m}, 1 \mathrm{H}), 2.10-1.90(\mathrm{~m}, 11 \mathrm{H}), 1.69-1.66(\mathrm{~m}, 2 \mathrm{H}), 1.46-1.41(\mathrm{~m}, 1 \mathrm{H})$, $1.16(\mathrm{~s}, 9 \mathrm{H}), 0.87(\mathrm{~d}, J=6.6 \mathrm{~Hz}, 3 \mathrm{H}) ;{ }^{13} \mathrm{C} \mathrm{NMR}\left(100 \mathrm{MHz}, \mathrm{CDCl}_{3}\right): \delta=179.1,131.1,130.8,129.1$, $128.5,111.4,104.2,78.9,76.3,75.9,68.8,64.8,63.6,38.7,35.7,33.9,31.0,30.0,28.7,28.0,27.2,23.4$, 21.4, 15.5; HRMS (MALDI-FTMS) for $\mathrm{C}_{26} \mathrm{H}_{40} \mathrm{O}_{7} \mathrm{Na}^{+}\left[\mathrm{M}+\mathrm{Na}^{+}\right]$calcd 487.2666, found 487.2663.

Carboxylic Acid 25. To a solution of oxalyl chloride $\left(0.12 \mathrm{~mL}, 1.35 \mathrm{mmol}, 5.0\right.$ equiv) in $\mathrm{CH}_{2} \mathrm{Cl}_{2}(6$ $\mathrm{mL})$ at $-78{ }^{\circ} \mathrm{C}$ was added DMSO (0.19 mmol, $3.0 \mathrm{mmol}, 11.0$ equiv). The solution was allowed to stir

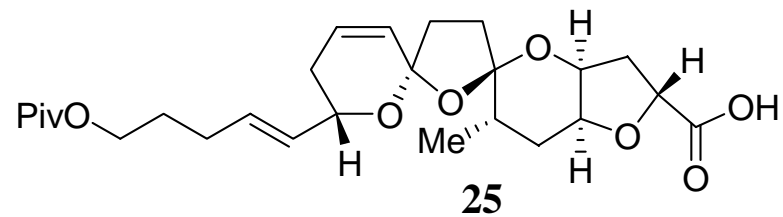

25 for $20 \mathrm{~min}$ at $-78{ }^{\circ} \mathrm{C}$ and then a solution of primary alcohol 23 (126 mg, $0.27 \mathrm{mmol}, 1.0$ equiv) in $\mathrm{CH}_{2} \mathrm{Cl}_{2}$ (5 $\mathrm{mL}$ ) was added. The resulting reaction mixture was allowed to stir at $-78^{\circ} \mathrm{C}$ for $1 \mathrm{~h}$, at which time $\mathrm{Et}_{3} \mathrm{~N}(0.86 \mathrm{~mL}, 6.0 \mathrm{mmol}, 22.0$ equiv) was added and the reaction mixture was allowed to warm to $0{ }^{\circ} \mathrm{C}$ over $1 \mathrm{~h}$. The reaction mixture was then quenched with saturated aqueous $\mathrm{NaCl}(15 \mathrm{~mL})$ and then extracted with $\mathrm{CH}_{2} \mathrm{Cl}_{2}(3 \times 10 \mathrm{~mL})$. The combined organic extracts were dried $\left(\mathrm{Na}_{2} \mathrm{SO}_{4}\right)$ and concentrated, and the obtained aldehyde 24 was used in the next step without further purification. Aldehyde 24 (116 mg, $0.25 \mathrm{mmol}, 1.0$ equiv) was dissolved in $t-\mathrm{BuOH}: \mathrm{H}_{2} \mathrm{O}$ $(4: 1,5 \mathrm{~mL})$ at $25^{\circ} \mathrm{C}$, and to the solution was added $\mathrm{NaClO}_{2}\left(135 \mathrm{mg}, 1.5 \mathrm{mmol}, 6.0\right.$ equiv), $\mathrm{NaH}_{2} \mathrm{PO}_{4}$ (234 mg, $1.5 \mathrm{mmol}, 6.0$ equiv), and 2-methyl-2-butene (0.27 mL, $2.5 \mathrm{mmol}, 10.0$ equiv). The reaction mixture was allowed to stir at $25^{\circ} \mathrm{C}$ for $1.5 \mathrm{~h}$, then quenched with a $\mathrm{pH} 7$ buffered saline solution $(5$ $\mathrm{mL})$ and then extracted with EtOAc $(3 \times 4 \mathrm{~mL})$. The combined organic extracts were dried $\left(\mathrm{Na}_{2} \mathrm{SO}_{4}\right)$ and concentrated to afford carboxylic acid 25 (110 mg, $0.23 \mathrm{mmol}, 85 \%$ over two steps) as a colorless oil. 25: $R_{\mathrm{f}}=0.11$ (silica gel, EtOAc: $\left.\mathrm{MeOH} 9: 1\right) ;[\alpha]_{\mathrm{D}}=-31.5\left(c=2.00, \mathrm{CHCl}_{3}\right)$; IR $($ film $) v_{\max }=3436$, 2960, 1727, 1456, 1396, 1320, 1285, $1090 \mathrm{~cm}^{-1} ;{ }^{1} \mathrm{H}$ NMR $\left(600 \mathrm{MHz}, \mathrm{CDCl}_{3}\right): \delta=5.99(\mathrm{ddd}, J=9.9$, 5.7, 2.2 Hz, $1 \mathrm{H}), 5.72-5.70(\mathrm{~m}, 1 \mathrm{H}), 5.69-5.65(\mathrm{~m}, 1 \mathrm{H}), 5.50(\mathrm{dd}, J=15.4,6.1 \mathrm{~Hz}, 1 \mathrm{H}), 4.85(\mathrm{dd}, J=$ 9.0, $8.0 \mathrm{~Hz}, 1 \mathrm{H}), 4.41$ (ddd, $J=11.8,5.1,4.4 \mathrm{~Hz}, 1 \mathrm{H}), 4.23-4.19(\mathrm{~m}, 1 \mathrm{H}), 4.03$ (t, J = 7.0 Hz, $2 \mathrm{H})$, 4.02-3.97 (m, 1 H), $2.54(\mathrm{dd}, J=13.6,7.4 \mathrm{~Hz}, 1 \mathrm{H}), 2.24-1.95(\mathrm{~m}, 11 \mathrm{H}), 1.72-1.68(\mathrm{~m}, 2 \mathrm{H}), 1.50-$ $1.46(\mathrm{~m}, 1 \mathrm{H}), 1.19(\mathrm{~s}, 9 \mathrm{H}), 0.91(\mathrm{~d}, J=6.6 \mathrm{~Hz}, 3 \mathrm{H}) ;{ }^{13} \mathrm{C} \mathrm{NMR}\left(100 \mathrm{MHz}, \mathrm{CDCl}_{3}\right): \delta=178.6,131.2$ 
$130.8,128.8,128.7,111.4,104.3,77.6,76.0,75.4,68.9,63.6,38.5,35.6,33.2,30.7,30.0,28.7,28.0$, 27.19, 27.18, 23.5, 15.5; HRMS (MALDI-FTMS) for $\mathrm{C}_{26} \mathrm{H}_{38} \mathrm{O}_{8} \mathrm{Na}^{+}\left[\mathrm{M}+\mathrm{Na}^{+}\right]$calcd 501.2459, found 501.2467

Dithiane coupling product 26. To a solution of carboxylic acid 25 (122 mg, $0.25 \mathrm{mmol}, 1.0$ equiv) in $\mathrm{CH}_{2} \mathrm{Cl}_{2}(3 \mathrm{~mL})$ at $25{ }^{\circ} \mathrm{C}$ was added DCC (103 mg, $0.50 \mathrm{mmol}, 2.0$ equiv) followed by pentafluorophenol

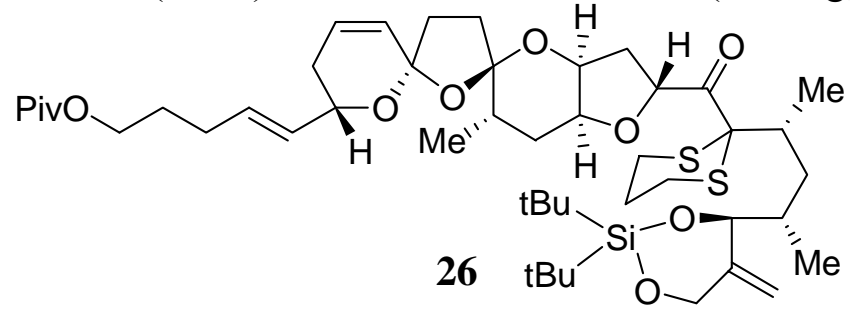

(69 $\mathrm{mg}, 0.38 \mathrm{mmol}, 1.5$ equiv). The reaction mixture was allowed to stir at $25{ }^{\circ} \mathrm{C}$ for $2.5 \mathrm{~h}$, then filtered through a plug of celite and concentrated, and the obtained crude PFP ester 7 was used in the next step without further purification. To a solution of dithiane 8 (734 mg, $1.76 \mathrm{mmol}, 9.0$ equiv) in THF (5 mL) at $25{ }^{\circ} \mathrm{C}$ was added a mixture of $n-\mathrm{BuLi}-n-\mathrm{Bu}_{2} \mathrm{Mg}(1.06 \mathrm{~mL}, 1.1 \mathrm{M}$ in hexanes, $1.17 \mathrm{mmol}, 6.0$ equiv), and the solution was allowed to stir for $1.5 \mathrm{~h}$ at $25{ }^{\circ} \mathrm{C}$, and then cooled to $-90{ }^{\circ} \mathrm{C}$. To the dithiane solution was added a solution of PFP ester 7 (120 mg, $0.205 \mathrm{mmol}, 1.0$ equiv) in THF (2 mL), and the reaction mixture was allowed to stir at $-90{ }^{\circ} \mathrm{C}$ for $15 \mathrm{~min}$ and then quenched with saturated aqueous $\mathrm{NaHCO}_{3}(5 \mathrm{~mL})$. The resulting biphasic mixture was extracted with EtOAc $(3 \times 5 \mathrm{~mL})$ and the combined organic extracts were dried $\left(\mathrm{Na}_{2} \mathrm{SO}_{4}\right)$, concentrated and then purified by flash column chromatography (silica gel, EtOAc:hexanes $1: 9 \rightarrow 1: 1)$ to provide coupling product 26 (113 mg, 0.13 mmol, 52\% over two steps) as a colorless oil. 26: $R_{\mathrm{f}}=0.52$ (silica gel, EtOAc:hexanes $\left.2: 1\right) ;[\alpha]_{\mathrm{D}}=$ $+53.2\left(c=3.00, \mathrm{CHCl}_{3}\right)$; IR (film) $v_{\max }=2933,2860,1728,1707,1515,1467,1320,1284,1157,1084$ $\mathrm{cm}^{-1} ;{ }^{1} \mathrm{H}$ NMR $\left(600 \mathrm{MHz}, \mathrm{CDCl}_{3}\right): \delta=5.97(\mathrm{ddd}, J=10.1,5.7,1.7 \mathrm{~Hz}, 1 \mathrm{H}), 5.69-5.64(\mathrm{~m}, 2 \mathrm{H}), 5.50$ $(\mathrm{dd}, J=15.3,6.2 \mathrm{~Hz}, 1 \mathrm{H}), 5.36(\mathrm{dd}, J=9.0,6.6 \mathrm{~Hz}, 1 \mathrm{H}), 5.10(\mathrm{~s}, 1 \mathrm{H}), 4.95(\mathrm{~s}, 1 \mathrm{H}), 4.55$ ( d, $J=11.4$ $\mathrm{Hz}, 1 \mathrm{H}), 4.40$ (ddd, $J=10.5,5.0,4.9 \mathrm{~Hz}, 1 \mathrm{H}), 4.25$ (d, $J=6.5 \mathrm{~Hz}, 1 \mathrm{H}), 4.23$ (br s, $1 \mathrm{H}), 4.17$ (d, $J=$ $11.8 \mathrm{~Hz}, 1 \mathrm{H}), 4.16(\mathrm{br} \mathrm{s}, 1 \mathrm{H}), 4.03(\mathrm{t}, J=6.6 \mathrm{~Hz}, 2 \mathrm{H}), 2.91(\mathrm{t}, J=13.6 \mathrm{~Hz}, 1 \mathrm{H}), 2.85(\mathrm{t}, J=12.7 \mathrm{~Hz}$ $1 \mathrm{H}), 2.59(\mathrm{tt}, J=14.5,4.0 \mathrm{~Hz}, 2 \mathrm{H}), 2.29(\mathrm{dd}, J=12.7,6.6 \mathrm{~Hz}, 2 \mathrm{H}), 2.25-2.18(\mathrm{~m}, 3 \mathrm{H}), 2.12-1.96(\mathrm{~m}$, $10 \mathrm{H}), 1.92-1.86(\mathrm{~m}, 1 \mathrm{H}), 1.76-1.68(\mathrm{~m}, 1 \mathrm{H}), 1.69$ (t, J=7.0 Hz, $2 \mathrm{H}), 1.45$ (dt, J=13.8, 3.1 Hz, $1 \mathrm{H})$, 1.37-1.32 (m, $1 \mathrm{H}), 1.18(\mathrm{~s}, 9 \mathrm{H}), 1.12(\mathrm{~d}, J=6.5 \mathrm{~Hz}, 3 \mathrm{H}), 1.04(\mathrm{~s}, 9 \mathrm{H}), 0.98(\mathrm{~s}, 9 \mathrm{H}), 0.88(\mathrm{~d}, J=6.6$ 
$\mathrm{Hz}, 3 \mathrm{H}), 0.87(\mathrm{~d}, J=7.0 \mathrm{~Hz}, 3 \mathrm{H}) ;{ }^{13} \mathrm{C} \mathrm{NMR}\left(150 \mathrm{MHz}, \mathrm{CDCl}_{3}\right): \delta=204.6,178.7,145.8,131.2,130.8$, $129.0,128.5,114.4,111.6,104.2,80.7,77.6,76.3,76.0,69.4,68.8,67.7,63.6,40.5,38.7,38.4,36.3$, $35.7,35.1,33.3,30.9,30.7,30.0,28.6,28.0,27.3,27.3,27.2,27.0,24.6,23.3,21.5,21.0,17.2,16.7$, 15.5; HRMS (MALDI-FTMS) for $\mathrm{C}_{47} \mathrm{H}_{76} \mathrm{O}_{9} \mathrm{~S}_{2} \mathrm{SiNa}^{+}\left[\mathrm{M}+\mathrm{Na}^{+}\right]$calcd 899.4597, found 899.4601.

Tetraol 28. To a solution of dithiane coupling product 26 (105 mg, $0.12 \mathrm{mmol}, 1.0$ equiv) in $\mathrm{CH}_{2} \mathrm{Cl}_{2}(3$ $\mathrm{mL}$ ) cooled to $-90{ }^{\circ} \mathrm{C}$ was added DIBAL-H (1.2 mL, $1.0 \mathrm{M}$ in $\mathrm{CH}_{2} \mathrm{Cl}_{2}, 1.2 \mathrm{mmol}$, 10 equiv), and the

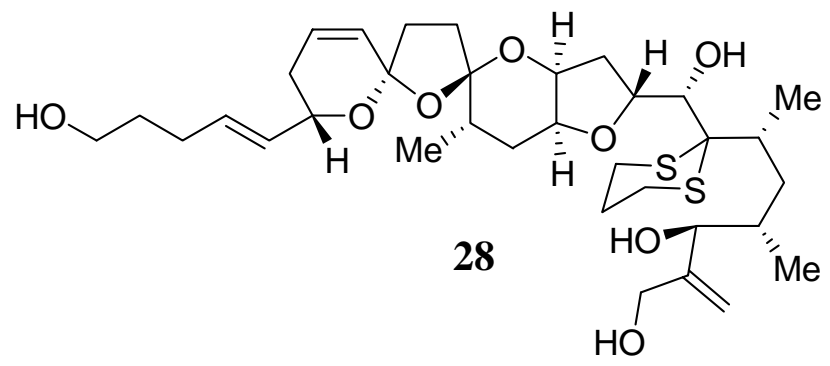
reaction mixture was maintained at $-90^{\circ} \mathrm{C}$ for $1.5 \mathrm{~h}$ at which time it was quenched with $\mathrm{MeOH}(0.3 \mathrm{~mL})$, saturated aqueous sodium and potassium tartrate (5 $\mathrm{mL})$ and $\mathrm{H}_{2} \mathrm{O}(2 \mathrm{~mL})$ and allowed to warm to $25^{\circ} \mathrm{C}$ over $2 \mathrm{~h}$. The resulting biphasic mixture was then extracted with $\mathrm{CH}_{2} \mathrm{Cl}_{2}(3 \times 5 \mathrm{~mL})$ and the combined organic extracts were dried $\left(\mathrm{Na}_{2} \mathrm{SO}_{4}\right)$ and concentrated, and the obtained reduced product 27 was used in the next step without further purification. To a solution of reduced product 27 (92 mg, $0.12 \mathrm{mmol}, 1.0$ equiv) in THF $(5 \mathrm{~mL})$ at $25{ }^{\circ} \mathrm{C}$ was added TBAF $(0.60 \mathrm{~mL}, 1.0 \mathrm{M}$ in THF, $0.60 \mathrm{mmol}, 5.0$ equiv $)$ and the reaction mixture was allowed to stir at 25 ${ }^{\circ} \mathrm{C}$ for $16 \mathrm{~h}$. When complete, the reaction mixture was quenched with saturated aqueous $\mathrm{NH}_{4} \mathrm{Cl}(5 \mathrm{~mL})$ and then extracted with EtOAc $(3 \times 5 \mathrm{~mL})$. The combined organic extracts were dried $\left(\mathrm{Na}_{2} \mathrm{SO}_{4}\right)$, concentrated, and then purified by flash column chromatography (silica gel, EtOAc:hexanes 4:1) to afford tetraol 28 (33.8 mg, $0.051 \mathrm{mmol}, 43 \%$ over two steps) as a colorless oil. 28: $R_{\mathrm{f}}=0.10$ (silica gel, EtOAc:hexanes 4:1); $[\alpha]_{\mathrm{D}}=-14.8\left(c=1.00, \mathrm{CHCl}_{3}\right)$; IR (film) $v_{\max }=3357,2960,2922,2873,1733$, $1460,1381,1251,1143,1095 \mathrm{~cm}^{-1} ;{ }^{1} \mathrm{H}$ NMR $\left(600 \mathrm{MHz}, \mathrm{CDCl}_{3}\right): \delta=5.97-5.93(\mathrm{~m}, 1 \mathrm{H}), 5.71-5.63(\mathrm{~m}$, $2 \mathrm{H}), 5.49$ (dd, $J=14.9,6.2 \mathrm{~Hz}, 1 \mathrm{H}), 5.03$ (s, $1 \mathrm{H}), 4.98-4.94$ (m, $1 \mathrm{H}), 4.94$ (s, $1 \mathrm{H}), 4.38-4.36$ (m, 1 H), $4.28(\mathrm{~d}, J=12.7 \mathrm{~Hz}, 1 \mathrm{H}), 4.16(\mathrm{~s}, 1 \mathrm{H}), 4.04-3.92(\mathrm{~m}, 5 \mathrm{H}), 3.61(\mathrm{dd}, J=7.2,6.6 \mathrm{~Hz}, 2 \mathrm{H}), 3.10$ $(\mathrm{dd}, J=7.0,5.3 \mathrm{~Hz}, 1 \mathrm{H}), 3.10-2.90(\mathrm{~m}, 2 \mathrm{H}), 2.84(\mathrm{dd}, J=13.1,12.5 \mathrm{~Hz}, 1 \mathrm{H}), 2.63(\mathrm{~d}, J=14.5 \mathrm{~Hz}, 1$ H), $2.58(\mathrm{~d}, J=14.5 \mathrm{~Hz}, 1 \mathrm{H}), 2.46-2.38(\mathrm{~m}, 1 \mathrm{H}), 2.29(\mathrm{dd}, J=14.0,6.2 \mathrm{~Hz}, 1 \mathrm{H}), 2.24(\mathrm{dd}, J=13.1$, $5.3 \mathrm{~Hz}, 1 \mathrm{H}), 2.19-1.90(\mathrm{~m}, 18 \mathrm{H}), 1.29(\mathrm{~d}, J=6.5 \mathrm{~Hz}, 3 \mathrm{H}), 0.88(\mathrm{~d}, J=6.5 \mathrm{~Hz}, 3 \mathrm{H}), 0.75(\mathrm{~d}, J=6.6$ 
$\mathrm{Hz}, 3 \mathrm{H}) ;{ }^{13} \mathrm{C} \mathrm{NMR}\left(150 \mathrm{MHz}, \mathrm{CDCl}_{3}\right): \delta=149.3,132.5,125.6,113.8,111.7,104.6,83.3,77.9,76.2$, $74.8,69.2,64.3,63.6,62.5,59.4,40.4,40.1,36.1,35.1,34.2,32.3,31.5,30.4,29.0,27.0,24.8,24.5$, 23.9, 20.2, 17.1, 15.8, 14.0; HRMS (MALDI-FTMS) for $\mathrm{C}_{34} \mathrm{H}_{54} \mathrm{O}_{8} \mathrm{~S}_{2} \mathrm{Na}^{+}\left[\mathrm{M}+\mathrm{Na}^{+}\right]$calcd 677.3152, found 677.3131 .

Triacetate 29. Tetraol 28 (90 mg, $0.14 \mathrm{mmol}, 1.0$ equiv) was dissolved in a mixture of pyridine: $\mathrm{CH}_{2} \mathrm{Cl}_{2}$ $(1: 1,5 \mathrm{~mL})$ and cooled to $0{ }^{\circ} \mathrm{C}$. Acetic anhydride $(0.66 \mathrm{~mL}, 7.0 \mathrm{mmol}, 50$ equiv) was added dropwise

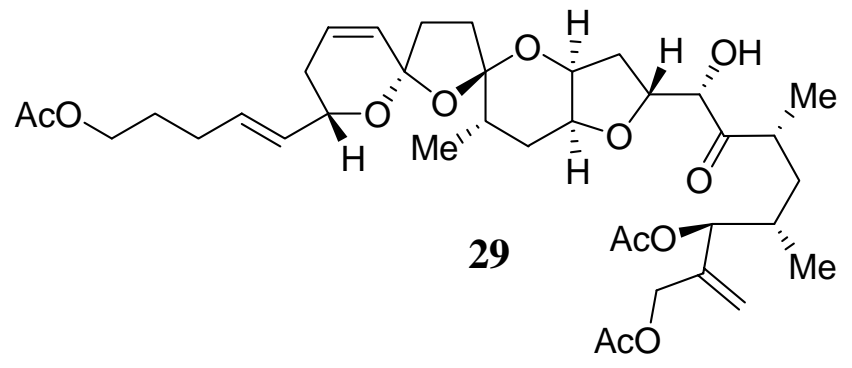
and the reaction mixture was stirred at $25^{\circ} \mathrm{C}$ for $16 \mathrm{~h}$ and then quenched slowly with saturated aqueous $\mathrm{NaHCO}_{3}(5 \mathrm{~mL})$. The aqueous layer was extracted with EtOAc $(3 \times 10 \mathrm{~mL})$ and the combined organic layers were dried over $\mathrm{Na}_{2} \mathrm{SO}_{4}$, filtered and concentrated. The crude product was dissolved in a mixture of MeCN:pH 7 buffered saline solution (4:1. $5 \mathrm{~mL})$ and cooled to $0{ }^{\circ} \mathrm{C}$. $\mathrm{PhI}\left(\mathrm{OCOCF}_{3}\right)_{2}(0.52 \mathrm{~mL}, 0.5 \mathrm{M}$ in $\mathrm{MeCN}, 0.26 \mathrm{mmol}, 2.2$ equiv $)$ was added and the reaction mixture was stirred at $0{ }^{\circ} \mathrm{C}$ for $10 \mathrm{~min}$ and quenched with saturated aqueous $\mathrm{Na}_{2} \mathrm{~S}_{2} \mathrm{O}_{3}(5$ $\mathrm{mL})$. The aqueous layer was extrated with EtOAc $(3 \times 10 \mathrm{~mL})$, and the combined organic layers were washed with water $(20 \mathrm{~mL})$ and brine $(30 \mathrm{~mL})$ and dried over $\mathrm{Na}_{2} \mathrm{SO}_{4}$. The crude mixture was purified by flash column chromatography (silica gel, EtOAc:hexanes 1:1) to afford triacetate 29 (64 mg, 0.092 mmol, 66\% over two steps) as a colorless oil. 29: $R_{\mathrm{f}}=0.15$ (silica gel, EtOAc:hexanes 1:1); $[\alpha]_{\mathrm{D}}=-$ $1.70\left(c=0.9, \mathrm{CHCl}_{3}\right)$; IR (film) $v_{\max }=3448,2954,2925,1738,1455,1370,1236,1090 \mathrm{~cm}^{-1} ;{ }^{1} \mathrm{H} \mathrm{NMR}$ $\left(600 \mathrm{MHz}, \mathrm{CDCl}_{3}\right): \delta=5.99(\mathrm{ddd}, J=11.7,6.6,3.1 \mathrm{~Hz}, 1 \mathrm{H}), 5.72-5.64(\mathrm{~m}, 2 \mathrm{H}), 5.51(\mathrm{dd}, J=18.4$, $7.5 \mathrm{~Hz}, 1 \mathrm{H}), 5.30(\mathrm{~s}, 1 \mathrm{H}), 5.17(\mathrm{~s}, 1 \mathrm{H}), 5.11(\mathrm{~d}, J=8.0 \mathrm{~Hz}, 1 \mathrm{H}), 4.84(\mathrm{t}, J=11.0 \mathrm{~Hz}, 1 \mathrm{H}), 4.63(\mathrm{~d}, J$ $=16.3 \mathrm{~Hz}, 1 \mathrm{H}), 4.56(\mathrm{~d}, J=16.3 \mathrm{~Hz}, 1 \mathrm{H}), 4.42-4.39(\mathrm{~m}, 1 \mathrm{H}), 4.23(\mathrm{dd}, J=6.4,2.2 \mathrm{~Hz}, 1 \mathrm{H}), 4.19$ (br s, $1 \mathrm{H}), 4.04(\mathrm{t}, J=7.9 \mathrm{~Hz}, 2 \mathrm{H}), 3.83$ (br s, $1 \mathrm{H}), 3.68$ (d, J=6.2 Hz, $1 \mathrm{H}), 2.94-2.91(\mathrm{~m}, 1 \mathrm{H}), 2.46-$ $2.39(\mathrm{~m}, 1 \mathrm{H}), 2.11(\mathrm{~s}, 3 \mathrm{H}), 2.09$ (s, $3 \mathrm{H}), 2.04$ (s, $3 \mathrm{H}), 2.15-1.90$ (m, 14 H), 1.73-1.67 (m, $1 \mathrm{H}), 1.40-$ $1.33(\mathrm{~m}, 1 \mathrm{H}), 1.11(\mathrm{~d}, J=8.3 \mathrm{~Hz}, 3 \mathrm{H}), 1.01-0.92(\mathrm{~m}, 1 \mathrm{H}), 0.86(\mathrm{~d}, J=7.9 \mathrm{~Hz}, 3 \mathrm{H}), 0.85(\mathrm{~d}, J=8.4$ $\mathrm{Hz}, 3 \mathrm{H}) ;{ }^{13} \mathrm{C} \mathrm{NMR}\left(150 \mathrm{MHz}, \mathrm{CDCl}_{3}\right): \delta=212.4,171.1,170.5,170.4,141.1,131.1,130.8,129.1$, 
128.6, 115.0, 111.3, 104.2, 78.6, 78.6, 78.5, 76.1, 75.6, 68.8, 64.1, 63.8, 38.4, 35.8, 35.7, 33.9, 33.9, $32.3,31.1,30.0,28.6,28.0,23.4,21.0,21.0,20.9,19.5,16.3,15.5$; HRMS (MALDI-FTMS) for $\mathrm{C}_{37} \mathrm{H}_{54} \mathrm{O}_{12} \mathrm{Na}^{+}\left[\mathrm{M}+\mathrm{Na}^{+}\right]$calcd 713.3507, found 713.3491.

Stille coupling product 30 . Triacetate $29(50 \mathrm{mg}, 0.072 \mathrm{mmol}, 1.0$ equiv) was azeotroped with benzene $(3 \times 5 \mathrm{~mL})$ and then dissolved in NMP $(2.5 \mathrm{~mL})$ at $25^{\circ} \mathrm{C}$. $\mathrm{LiCl}\left(19 \mathrm{mg}, 0.44 \mathrm{mmol}, 6.0\right.$ equiv), $i-\mathrm{Pr}_{2} \mathrm{NEt}$

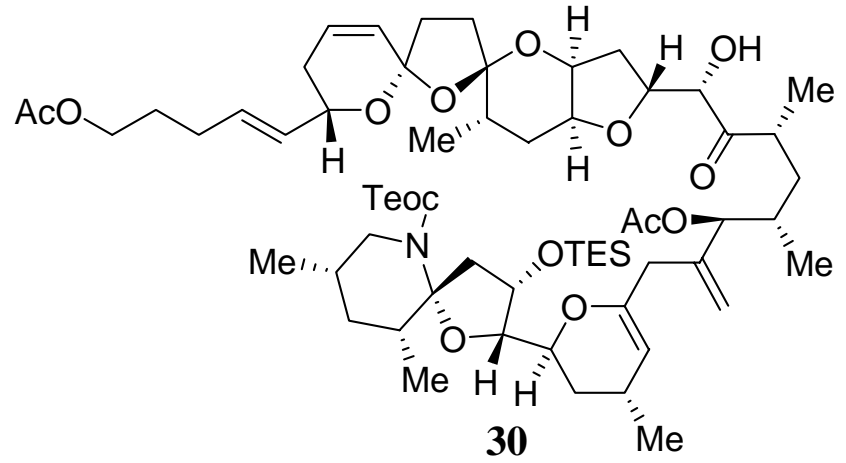
(0.075 mL, $0.44 \mathrm{mmol}, 6.0$ equiv) and $\mathrm{AsPh}_{3}(7 \mathrm{mg}$, $0.022 \mathrm{mmol}, 0.3$ equiv) were added at $25^{\circ} \mathrm{C}$ followed by $\mathrm{Pd}_{2} \mathrm{dba}_{3}$ (20 mg, $0.022 \mathrm{mmol}, 0.3$ equiv). The reaction mixture was heated to $45{ }^{\circ} \mathrm{C}$, and to this solution was added a solution of vinyl stannane $\mathbf{6}$ (153 mg, $0.22 \mathrm{mmol}, 3.0$ equiv) in THF $(2.5 \mathrm{~mL})$

over a period of $4 \mathrm{~h}$ via syringe pump. The reaction mixture was cooled to $25^{\circ} \mathrm{C}$ and quenched slowly with saturated aqueous $\mathrm{NaHCO}_{3}(2 \mathrm{~mL})$. The aqueous layer was extracted with EtOAc $(3 \times 10 \mathrm{~mL})$ and the combined organic layers were washed with brine $(10 \mathrm{~mL})$, dried over $\mathrm{Na}_{2} \mathrm{SO}_{4}$, and concentrated. The crude product was purified by flash column chromatography (silica gel, hexanes:EtOAc 3:1) to afford Stille coupling product $30(45 \mathrm{mg}, 0.037 \mathrm{mmol}, 52 \%)$ as a colorless oil. 30: $R_{\mathrm{f}}=0.40$ (silica gel, EtOAc:hexanes 1:1); $[\alpha]_{\mathrm{D}}=-2.40\left(c=0.50, \mathrm{CHCl}_{3}\right) ; \mathrm{IR}(\mathrm{film}) v_{\max }=2955,2361,1737,1698,1455$, 1396, 1240, 1167, $1068 \mathrm{~cm}^{-1} ;{ }^{1} \mathrm{H}$ NMR (500 MHz, $\left.\mathrm{CDCl}_{3}\right): \delta=5.99(\mathrm{ddd}, J=9.8,5.4,2.1 \mathrm{~Hz}, 1 \mathrm{H})$, 5.72-5.62 (m, $2 \mathrm{H}), 5.50(\mathrm{dd}, J=15.4,6.2 \mathrm{~Hz}, 1 \mathrm{H}), 5.08$ (dd, $J=15.4,5.9 \mathrm{~Hz}, 1 \mathrm{H}), 5.07$ (s, $1 \mathrm{H}), 5.00$ (s, $1 \mathrm{H}), 4.87-4.81(\mathrm{~m}, 1 \mathrm{H}), 4.57$ (d, $J=4.0 \mathrm{~Hz}, 1 \mathrm{H}), 4.50$ (dd, $J=13.0,6.8 \mathrm{~Hz}, 2 \mathrm{H}), 4.42-4.37$ (m, 1 H), 4.22 (dd, $J=5.5,1.8 \mathrm{~Hz}, 1 \mathrm{H}), 4.19$ (dd, $J=3.3,2.9 \mathrm{~Hz}, 1 \mathrm{H}), 4.14-4.08$ (m, $3 \mathrm{H}), 4.06-4.01$ (m, 4 H), 3.83 (s, $1 \mathrm{H}), 3.71$ (d, $J=5.5 \mathrm{~Hz}, 1 \mathrm{H}), 3.67$ (dd, $J=13.6,2.9 \mathrm{~Hz}, 2 \mathrm{H}), 3.12-3.06$ (m, $2 \mathrm{H}), 2.93$ (dd, $J=14.7,6.6 \mathrm{~Hz}, 1 \mathrm{H}), 2.70-2.60$ (m, $2 \mathrm{H}), 2.49-2.41$ (m, $2 \mathrm{H}), 2.08$ (s, $3 \mathrm{H}), 2.12-1.94$ (m, $8 \mathrm{H}$ ), 2.03 (s, 3 H), 1.72-1.66 (m, 6 H), 1.64-1.32 (m, 6 H), 1.10 (d, J = 7.0 Hz, 3 H), 0.98 (d, J = 7.0 Hz, 3 H), $0.97(\mathrm{~d}, J=7.0 \mathrm{~Hz}, 3 \mathrm{H}), 0.92(\mathrm{t}, J=7.9 \mathrm{~Hz}, 9 \mathrm{H}), 0.84(\mathrm{~d}, J=7.3 \mathrm{~Hz}, 3 \mathrm{H}), 0.83(\mathrm{~d}, J=6.6 \mathrm{~Hz}, 3$ H), 0.79 (d, $J=6.2 \mathrm{~Hz}, 3 \mathrm{H}), 0.78$ (d, $J=6.6 \mathrm{~Hz}, 3 \mathrm{H}), 0.57$ (q, $J=7.9 \mathrm{~Hz}, 6 \mathrm{H}), 0.03(\mathrm{~s}, 9 \mathrm{H}) ;{ }^{13} \mathrm{C} \mathrm{NMR}$ 
$\left(125 \mathrm{MHz}, \mathrm{CDCl}_{3}\right): \delta=213.4,171.2,157.1,151.7,144.1,132.1,131.8,130.1,129.7,129.6,114.2$, 112.3, 105.2, 105.0, 97.1, 84.0, 81.3, 79.5, 78.2, 77.1, 76.5, 73.0, 72.1, 69.8, 64.8, 63.8, 49.9, 43.9, 39.5, $39.2,38.7,38.2,36.9,36.7,35.0,34.7,32.8,32.1,32.1,31.8,31.0,30.7,29.7,29.2,29.0,25.5,24.4$, 23.8, 22.1, 22.0, 20.5, 19.6, 17.7, 17.4, 16.5, 7.8, 5.6, -0.5; HRMS (MALDI-FTMS) for $\mathrm{C}_{63} \mathrm{H}_{103} \mathrm{NO}_{15} \mathrm{Si}_{2} \mathrm{Na}^{+}\left[\mathrm{M}+\mathrm{Na}^{+}\right]$calcd 1192.6758, found 1192.6724.

FGHI Diastereomeric Stille Coupling Product 31. Triacetate 29 (50 mg, $0.072 \mathrm{mmol}, 1.0$ equiv) was azeotroped with benzene $(3 \times 5 \mathrm{~mL})$ and then dissolved in NMP $(2.5 \mathrm{~mL})$ at $25{ }^{\circ} \mathrm{C}$. $\mathrm{LiCl}(19 \mathrm{mg}, 0.44$

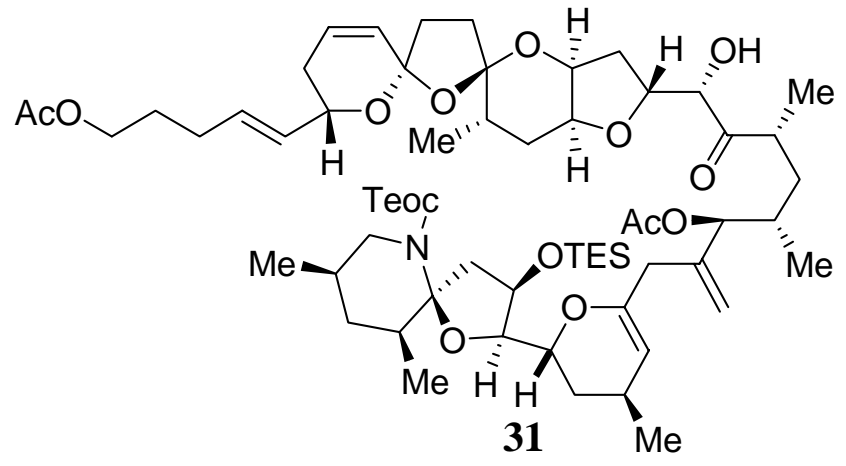
mmol, 6.0 equiv), i-Pr 2 NEt $(0.075 \mathrm{~mL}, 0.44 \mathrm{mmol}$, 6.0 equiv) and $\mathrm{AsPh}_{3}(7 \mathrm{mg}, 0.022 \mathrm{mmol}, 0.3$ equiv) were added at $25{ }^{\circ} \mathrm{C}$ followed by $\operatorname{Pd}_{2} \mathrm{dba}_{3}(20 \mathrm{mg}$, $0.022 \mathrm{mmol}, 0.3$ equiv). The reaction mixture was then heated to $45^{\circ} \mathrm{C}$ and to this solution was added dropwise a solution of vinyl stannane ent-6 (153 mg, $0.22 \mathrm{mmol}, 3.0$ equiv) in THF (2.5 mL) over a period of $4 \mathrm{~h}$ via syringe pump. The reaction mixture was cooled to $25^{\circ} \mathrm{C}$ and quenched slowly with saturated aqueous $\mathrm{NaHCO}_{3}(2 \mathrm{~mL})$. The aqueous layer was extrated with EtOAc $(3 \times 10 \mathrm{~mL})$ and the combined organic layers were washed with brine $(10 \mathrm{~mL})$, dried over $\mathrm{Na}_{2} \mathrm{SO}_{4}$, and concentrated. The crude product was purified by flash column chromatography (silica gel, hexanes:EtOAc 3:1) to afford FGHI diastereomeric Stille coupling product 31 (47 mg, 0.040 mmol, 56\%) as a colorless oil. 31: $R_{\mathrm{f}}=0.55$ (silica gel, EtOAc:hexanes 1:1); $[\alpha]_{\mathrm{D}}=+5.70(c=1.10$, $\mathrm{CHCl}_{3}$ ); IR (film) $v_{\max }=2955,2320,1740,1696,1455,1390,1241,1173,1069 \mathrm{~cm}^{-1} ;{ }^{1} \mathrm{H}$ NMR (600 $\left.\mathrm{MHz}, \mathrm{CDCl}_{3}\right): \delta=5.99(\mathrm{dd}, J=8.8,4.8 \mathrm{~Hz}, 1 \mathrm{H}), 5.70(\mathrm{~d}, J=11.0 \mathrm{~Hz}, 1 \mathrm{H}), 5.66(\mathrm{dd}, J=14.7,7.2 \mathrm{~Hz}$ $1 \mathrm{H}), 5.51(\mathrm{dd}, J=15.3,5.7 \mathrm{~Hz}, 1 \mathrm{H}), 5.12(\mathrm{~d}, J=5.3 \mathrm{~Hz}, 1 \mathrm{H}), 5.05(\mathrm{~s}, 1 \mathrm{H}), 5.00(\mathrm{~s}, 1 \mathrm{H}), 4.84(\mathrm{dd}, J=$ 7.9, $7.5 \mathrm{~Hz}, 1 \mathrm{H}), 4.54(\mathrm{~d}, J=3.5 \mathrm{~Hz}, 1 \mathrm{H}), 4.47$ (dd, $J=11.8,6.1 \mathrm{~Hz}, 1 \mathrm{H}), 4.40$ (br s, $1 \mathrm{H}), 4.22(\mathrm{~d}, J=$ $4.8 \mathrm{~Hz}, 1 \mathrm{H}), 4.19$ (br s, $1 \mathrm{H}), 4.13-4.01(\mathrm{~m}, 6 \mathrm{H}), 3.83(\mathrm{~s}, 1 \mathrm{H}), 3.71$ (d, J=5.3 Hz, $1 \mathrm{H}), 3.67$ (d, $J=$ $10.1 \mathrm{~Hz}, 2 \mathrm{H}), 3.07$ (t, $J=12.5 \mathrm{~Hz}, 1 \mathrm{H}), 3.04-2.96(\mathrm{~m}, 2 \mathrm{H}), 2.79(\mathrm{~d}, J=15.8 \mathrm{~Hz}, 1 \mathrm{H}), 2.71(\mathrm{~s}, 1 \mathrm{H})$, $2.67(\mathrm{~d}, J=15.8 \mathrm{~Hz}, 1 \mathrm{H}), 2.49-2.37$ (m, $2 \mathrm{H}), 2.22$ (br s, $1 \mathrm{H}), 2.07$ (s, 3 H), 2.13-1.96 (m, $11 \mathrm{H}), 2.03$ 
(s, $3 \mathrm{H}), 1.91-1.65(\mathrm{~m}, 7 \mathrm{H}), 1.10(\mathrm{~d}, J=7.0 \mathrm{~Hz}, 3 \mathrm{H}), 0.99$ (d, J = 7.0 Hz, $3 \mathrm{H}), 1.02-0.90(\mathrm{~m}, 2 \mathrm{H})$, $0.92(\mathrm{t}, J=7.7 \mathrm{~Hz}, 9 \mathrm{H}), 0.84(\mathrm{~d}, J=6.6 \mathrm{~Hz}, 3 \mathrm{H}), 0.82(\mathrm{~d}, J=6.6 \mathrm{~Hz}, 3 \mathrm{H}), 0.80(\mathrm{~d}, J=6.1 \mathrm{~Hz}, 3 \mathrm{H})$, $0.79(\mathrm{~d}, J=6.1 \mathrm{~Hz}, 3 \mathrm{H}), 0.57(\mathrm{q}, J=7.7 \mathrm{~Hz}, 6 \mathrm{H}), 0.03(\mathrm{~s}, 9 \mathrm{H}) ;{ }^{13} \mathrm{C} \mathrm{NMR}\left(150 \mathrm{MHz}, \mathrm{CDCl}_{3}\right): \delta=$ $213.3,172.0,171.0,157.0,151.5,144.0,132.0,131.7,129.6,129.2,114.3,112.2,105.1,104.9,97.1$, 83.7, 80.8, 79.4, 78.1, 77.1, 76.4, 71.9, 69.7, 64.7, 63.7, 49.9, 43.7, 39.3, 39.0, 38.4, 38.3, 36.7, 36.5, $34.9,34.4,33.0,32.0,32.0,31.9,31.2,30.9,29.5,29.1,28.8,25.2,24.2,23.6,21.9,21.8,20.4,19.5$, 18.5, 17.6, 17.4, 16.4, 7.7, 5.5, -0.6; HRMS (MALDI-FTMS) for $\mathrm{C}_{63} \mathrm{H}_{103} \mathrm{NO}_{15} \mathrm{Si}_{2} \mathrm{Na}^{+}\left[\mathrm{M}+\mathrm{Na}^{+}\right]$calcd 1192.6758, found 1192.6705 .

Octacycle 33. Stille coupling product $30(60 \mathrm{mg}, 0.051 \mathrm{mmol}, 1.0$ equiv) was dissolved in a mixture of pyridine:THF $(1: 1,3 \mathrm{~mL})$ and cooled to $0{ }^{\circ} \mathrm{C}$. HF•py $(0.15 \mathrm{~mL})$ was added dropwise and the reaction

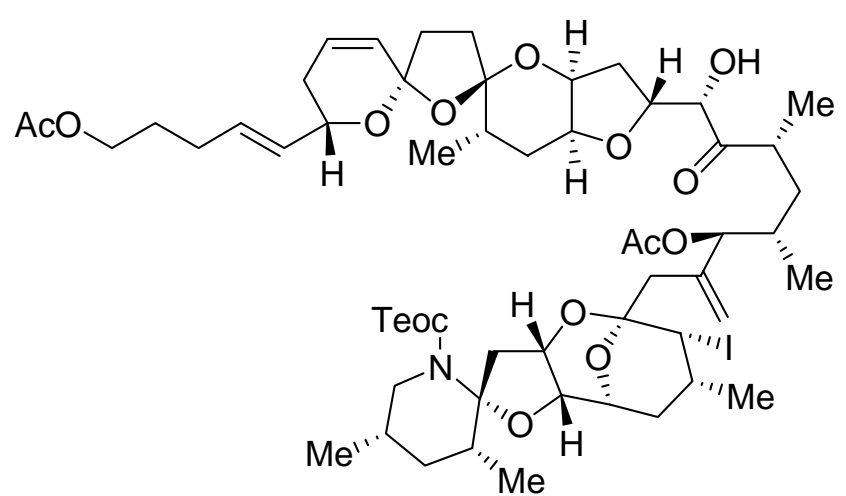

33 mixture was stirred at $0{ }^{\circ} \mathrm{C}$ for $2.5 \mathrm{~h}$ and then quenched slowly with saturated aqueous $\mathrm{NaHCO}_{3}(5$ $\mathrm{mL})$. The resulting biphasic mixture was extracted with EtOAc $(3 \times 10 \mathrm{~mL})$ and the combined organic layers were dried over $\mathrm{Na}_{2} \mathrm{SO}_{4}$, filtered, and concentrated. The crude product was dissolved in THF $(6 \mathrm{~mL})$ and cooled to $0{ }^{\circ} \mathrm{C} . \mathrm{NaHCO}_{3}(130 \mathrm{mg}$, $1.5 \mathrm{mmol}, 30$ equiv) was added followed by NIS (115 mg, $0.51 \mathrm{mmol}, 10$ equiv). The reaction mixture was stirred at $0{ }^{\circ} \mathrm{C}$ overnight and then quenched slowly with saturated aqueous $\mathrm{Na}_{2} \mathrm{~S}_{2} \mathrm{O}_{3}(5 \mathrm{~mL})$ at $0{ }^{\circ} \mathrm{C}$. The aqueous layer was extracted with EtOAc $(3 \times 10 \mathrm{~mL})$ and the combined organic layers were dried over $\mathrm{Na}_{2} \mathrm{SO}_{4}$, filtered, and concentrated. The crude product was purified by flash column chromatography (silica gel, hexanes:EtOAc 3:1) to afford octacycle 33 (43 mg, $0.034 \mathrm{mmol}, 67 \%$ over two steps) as a colorless oil. The oil was then crystallized from a 1:1 solution of $\mathrm{CH}_{2} \mathrm{Cl}_{2}$ : cyclohexane (1 $\mathrm{mL}$ ) to obtain colorless crystals to be used for X-ray analysis. 33: $R_{\mathrm{f}}=0.60$ (silica gel, EtOAc:hexanes 1:1); $\mathrm{mp}=140-150{ }^{\circ} \mathrm{C} ;[\alpha]_{\mathrm{D}}=-7.60\left(c=2.40, \mathrm{CHCl}_{3}\right) ; \mathrm{IR}($ film $) v_{\max }=2957,1734,1703,1455,1431$, 1358, 1243, 1176, $1067 \mathrm{~cm}^{-1} ;{ }^{1} \mathrm{H}$ NMR (600 MHz, $\left.\mathrm{CDCl}_{3}\right): \delta=5.99(\mathrm{ddd}, J=9.8,5.6,1.9 \mathrm{~Hz}, 1 \mathrm{H})$, 
$5.71(\mathrm{~d}, J=10.1 \mathrm{~Hz}, 1 \mathrm{H}), 5.68-5.62(\mathrm{~m}, 1 \mathrm{H}), 5.54(\mathrm{~s}, 1 \mathrm{H}), 5.50(\mathrm{dd}, J=15.6,6.4 \mathrm{~Hz}, 1 \mathrm{H}), 5.33(\mathrm{~s}, 1$ H), $5.06(\mathrm{~m}, 2 \mathrm{H}), 4.95-4.92(\mathrm{~m}, 1 \mathrm{H}), 4.59$ (dd, J = 18.0, 9.2 Hz, $1 \mathrm{H}), 4.43-4.39$ (m, $1 \mathrm{H}), 4.29$ (s, 1 H), $4.22(\mathrm{~d}, J=8.3 \mathrm{~Hz}, 1 \mathrm{H}), 4.19($ br s, $1 \mathrm{H}), 4.16-4.12(\mathrm{~m}, 3 \mathrm{H}), 4.04(\mathrm{t}, J=6.6 \mathrm{~Hz}, 2 \mathrm{H}), 3.99(\mathrm{~d}, J=$ $8.8 \mathrm{~Hz}, 1 \mathrm{H}), 3.86$ (m, $1 \mathrm{H}), 3.69-3.67$ (m, $1 \mathrm{H}), 3.15-3.08$ (m, $4 \mathrm{H}), 2.48-2.45$ (m, $2 \mathrm{H}), 2.10$ (s, $3 \mathrm{H})$, 2.10-2.06 (m, 5 H), 2.04 (s, $3 \mathrm{H}), 1.99-1.95$ (m, $5 \mathrm{H}), 1.92-1.89$ (m, $2 \mathrm{H}), 1.84-1.81$ (m, $2 \mathrm{H}), 1.71-$ 1.69 (m, 2 H), 1.41-1.29 (m, 4 H), 1.07 (d, J = 7.0 Hz, 3 H), 0.99-0.96 (m, 3 H), 0.93 (d, J = 6.6 Hz, 3 H), $0.88(\mathrm{~d}, J=7.0 \mathrm{~Hz}, 3 \mathrm{H}), 0.85(\mathrm{~d}, J=7.0 \mathrm{~Hz}, 3 \mathrm{H}), 0.79(\mathrm{~d}, J=6.6 \mathrm{~Hz}, 3 \mathrm{H}), 0.69$ (d, $J=6.6 \mathrm{~Hz}, 3$ $\mathrm{H}), 0.02(\mathrm{~s}, 9 \mathrm{H}) ;{ }^{13} \mathrm{C} \mathrm{NMR}\left(150 \mathrm{MHz}, \mathrm{CDCl}_{3}\right): \delta=212.1,172.3,171.7,157.2,140.8,132.1,131.8$, $130.1,129.3,118.0,112.2,105.2,98.7,97.9,80.6,80.4,80.0,77.6,77.4,76.9,76.7,73.9,69.9,64.8$, $64.2,50.7,50.4,47.3,42.0,38.1,36.7,35.7,34.1,33.1,32.2,32.0,31.4,31.0,29.7,29.2,29.1,29.0$, 27.4, 24.6, 24.4, 22.0, 22.0, 20.3, 19.5, 18.3, 17.4, 16.6, -0.3; HRMS (MALDI-FTMS) for $\mathrm{C}_{57} \mathrm{H}_{88} \mathrm{INO}_{15} \mathrm{SiNa}^{+}\left[\mathrm{M}+\mathrm{Na}^{+}\right]$calcd 1204.4860 , found 1204.4833 .

FGHI Diastereomeric Octacycle 34. FGHI diastereomeric Stille coupling product 31 (60 mg, 0.051 mmol, 1.0 equiv) was dissolved in a mixture of pyridine:THF $(1: 1,3 \mathrm{~mL})$ and cooled to $0{ }^{\circ} \mathrm{C}$. $\mathrm{HF} \bullet$ py

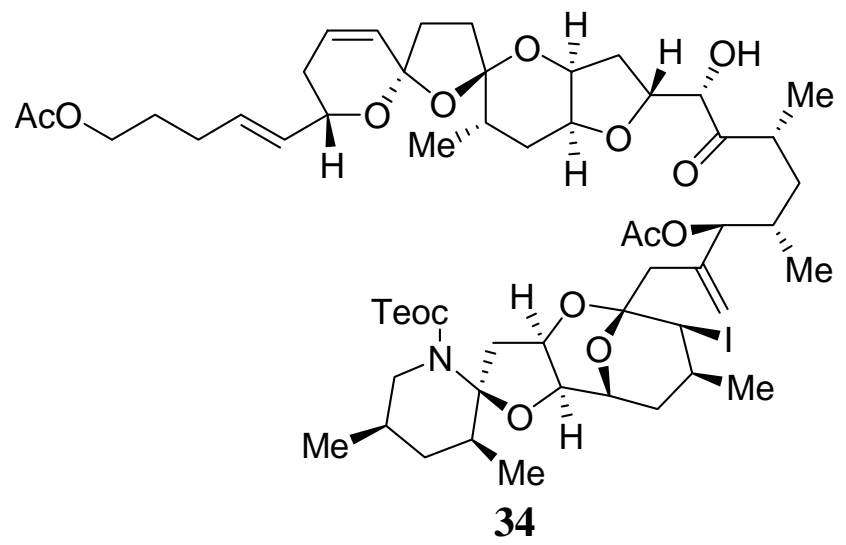
$(0.15 \mathrm{~mL})$ was added dropwise and the reaction mixture was stirred at $25{ }^{\circ} \mathrm{C}$ for $2.5 \mathrm{~h}$ and then quenched slowly with saturated aqueous $\mathrm{NaHCO}_{3}(5$ $\mathrm{mL})$ at $0{ }^{\circ} \mathrm{C}$. The aqueous layer was extracted with EtOAc $(3 \times 10 \mathrm{~mL})$ and the combined organic layers were dried over $\mathrm{Na}_{2} \mathrm{SO}_{4}$, filtered and concentrated. The crude product was dissolved in THF $(6 \mathrm{~mL})$ and cooled to $0{ }^{\circ} \mathrm{C} . \mathrm{NaHCO}_{3}(130 \mathrm{mg}, 1.5 \mathrm{mmol}, 30$ equiv) was added followed by NIS (115 mg, 0.51 mmol, 10 equiv). The reaction mixture was stirred at $0{ }^{\circ} \mathrm{C}$ overnight and then quenched slowly with saturated aqueous $\mathrm{Na}_{2} \mathrm{~S}_{2} \mathrm{O}_{3}(5 \mathrm{~mL})$ at $0{ }^{\circ} \mathrm{C}$. The aqueous layer was extracted with EtOAc $(3 \times 10 \mathrm{~mL})$ and the combined organic layers were dried over $\mathrm{Na}_{2} \mathrm{SO}_{4}$, filtered, and concentrated. The crude product was purified by flash column chromatography (silica gel, hexanes:EtOAc 3:1) to afford FGHI 
diastereomeric octacycle 34 (40 mg, $0.032 \mathrm{mmol}, 63 \%$ over two steps) as a colorless oil. $34: R_{\mathrm{f}}=0.50$ (silica gel, EtOAc:hexanes 1:1); $[\alpha]_{\mathrm{D}}=+2.80\left(c=1.20, \mathrm{CHCl}_{3}\right)$; IR (film) $v_{\max }=2956,1737,1713$, 1596, 1449, 1390, 1355, 1243, 1173, $1067 \mathrm{~cm}^{-1} ;{ }^{1} \mathrm{H}$ NMR $\left(600 \mathrm{MHz}, \mathrm{CDCl}_{3}\right): \delta=5.98(\mathrm{ddd}, J=9.8$, 6.2, $1.8 \mathrm{~Hz}, 1 \mathrm{H}), 5.70(\mathrm{~d}, J=9.6 \mathrm{~Hz}, 1 \mathrm{H}), 5.69-5.63(\mathrm{~m}, 1 \mathrm{H}), 5.50(\mathrm{dd}, J=15.4,6.1 \mathrm{~Hz}, 1 \mathrm{H}), 5.46$ (s, $1 \mathrm{H}), 5.29(\mathrm{~s}, 1 \mathrm{H}), 5.07(\mathrm{~s}, 2 \mathrm{H}), 4.84-4.82(\mathrm{~m}, 1 \mathrm{H}), 4.75(\mathrm{dd}, J=12.5,6.4 \mathrm{~Hz}, 1 \mathrm{H}), 4.66(\mathrm{br}, 1 \mathrm{H})$, 4.42-4.38 (m, 1 H), $4.23(\mathrm{~d}, J=5.7 \mathrm{~Hz}, 1 \mathrm{H}), 4.19-4.17(\mathrm{~m}, 3 \mathrm{H}), 4.04-4.02(\mathrm{~m}, 4 \mathrm{H}), 3.92-3.91(\mathrm{~m}, 2$ H), $3.76(\mathrm{dd}, J=13.4,4.2 \mathrm{~Hz}, 1 \mathrm{H}), 3.64(\mathrm{dd}, J=14.7,5.0 \mathrm{~Hz}, 1 \mathrm{H}), 3.19$ (t, $J=12.5 \mathrm{~Hz}, 1 \mathrm{H}), 3.01-$ 2.99 (m, $1 \mathrm{H}), 2.88$ (d, J=14.5 Hz, $1 \mathrm{H}), 2.69$ (dd, $J=22.4,7.5 \mathrm{~Hz}, 1 \mathrm{H}), 2.45-2.38$ (m, $2 \mathrm{H}), 2.15-2.08$ (m, $6 \mathrm{H}), 2.08$ (s, $3 \mathrm{H}), 2.03$ (s, $3 \mathrm{H}), 1.99-1.92$ (m, $6 \mathrm{H}), 1.90-1.85$ (m, $2 \mathrm{H}), 1.71-1.68$ (m, 3 H), $1.34-$ $1.24(\mathrm{~m}, 8 \mathrm{H}), 1.06(\mathrm{~d}, J=7.5 \mathrm{~Hz}, 3 \mathrm{H}), 1.06-0.96(\mathrm{~m}, 3 \mathrm{H}), 0.97(\mathrm{~d}, J=6.1 \mathrm{~Hz}, 3 \mathrm{H}), 0.82(\mathrm{~d}, J=7.5$ $\mathrm{Hz}, 3 \mathrm{H}), 0.81(\mathrm{~d}, J=7.0 \mathrm{~Hz}, 3 \mathrm{H}), 0.78(\mathrm{~d}, J=6.6 \mathrm{~Hz}, 3 \mathrm{H}), 0.02(\mathrm{~s}, 9 \mathrm{H}) ;{ }^{13} \mathrm{C} \mathrm{NMR}(150 \mathrm{MHz}$ $\left.\mathrm{CDCl}_{3}\right): \delta=212.5,172.1,171.5,157.4,140.4,132.2,131.8,130.1,129.6,116.3,112.4,105.1,98.5$ $98.2,81.0,79.9,79.6,78.1,77.2,76.4,74.8,74.3,69.8,64.8,64.0,52.6,50.1,44.8,41.4,39.5,39.2$, $37.7,36.8,36.7,35.1,32.9,32.6,32.2,32.1,31.9,31.0,30.7,29.7,29.0,28.9,25.4,24.4,22.0,22.0$, 20.6, 19.6, 18.5, 18.0, 17.4, 16.6, -0.5; HRMS (MALDI-FTMS) for $\mathrm{C}_{57} \mathrm{H}_{88} \mathrm{INO}_{15} \mathrm{SiNa}^{+}\left[\mathrm{M}+\mathrm{Na}^{+}\right]$calcd 1204.4860, found 1204.4832.

Des-iodo product 35. Octacycle $33(21 \mathrm{mg}, 0.018 \mathrm{mmol}, 1.0$ equiv) was dissolved in a mixture of $n$ $\mathrm{Bu}_{3} \mathrm{SnH}$ :toluene $(1: 2,1.5 \mathrm{~mL})$ and cooled to $0{ }^{\circ} \mathrm{C} . \mathrm{Et}_{3} \mathrm{~B}(1.0 \mathrm{M}$ in hexanes, 1 drop $)$ was added and the

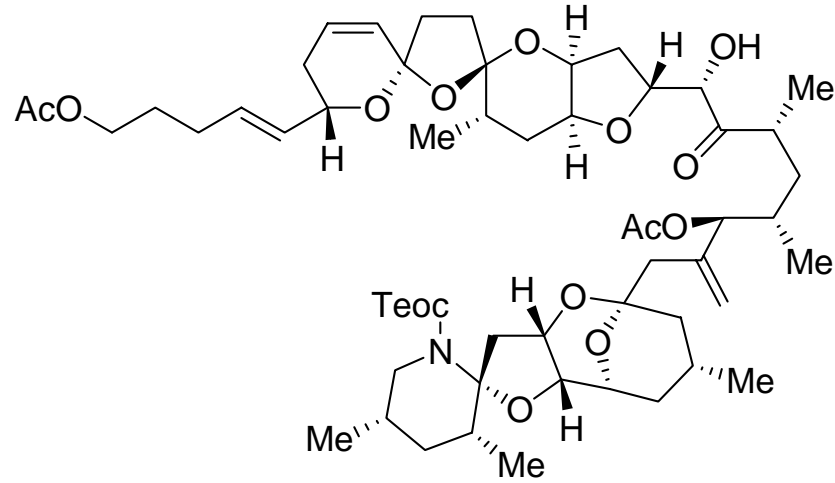

35 reaction mixture was stirred for $5 \mathrm{~min}$. The reaction mixture was then quenched with $\mathrm{H}_{2} \mathrm{O}(2 \mathrm{~mL})$. The aqueous layer was extracted with EtOAc $(3 \times 5 \mathrm{~mL})$ and the combined organic layers were washed with saturated aqueous $\mathrm{NaCl}(10 \mathrm{~mL})$, dried over $\mathrm{Na}_{2} \mathrm{SO}_{4}$, and concentrated. The crude product was purified by flash column chromatography (silica gel, hexanes:EtOAc 4:1) to afford des-iodo product $35(16 \mathrm{mg}, 0.017 \mathrm{mmol}, 92 \%)$ as a colorless oil. 35: $R_{\mathrm{f}}=$ 
0.30 (silica gel, EtOAc:hexanes 1:2); $[\alpha]_{\mathrm{D}}=-4.0\left(c=0.70, \mathrm{CHCl}_{3}\right)$; IR (film) $v_{\max }=2954,2914,2356$, 1735, 1690, 1458, 1397, 1250, $1175 \mathrm{~cm}^{-1} ;{ }^{1} \mathrm{H} \mathrm{NMR}\left(600 \mathrm{MHz}, \mathrm{CDCl}_{3}\right): \delta=5.98(\mathrm{ddd}, J=9.8,5.6,1.8$ $\mathrm{Hz}, 1 \mathrm{H}), 5.71(\mathrm{~d}, J=9.6 \mathrm{~Hz}, 1 \mathrm{H}), 5.67(\mathrm{dd}, J=15.1,7.2 \mathrm{~Hz}, 1 \mathrm{H}), 5.51(\mathrm{dd}, J=15.3,6.1 \mathrm{~Hz}, 1 \mathrm{H})$, $5.18(\mathrm{~d}, J=4.8 \mathrm{~Hz}, 1 \mathrm{H}), 5.15(\mathrm{~s}, 1 \mathrm{H}), 5.03(\mathrm{~s}, 1 \mathrm{H}), 4.85(\mathrm{dd}, J=8.8,7.5 \mathrm{~Hz}, 1 \mathrm{H}), 4.68(\mathrm{dd}, J=10.5$, $5.7 \mathrm{~Hz}, 1 \mathrm{H}), 4.42-4.37(\mathrm{~m}, 1 \mathrm{H}), 4.24(\mathrm{~d}, J=4.8 \mathrm{~Hz}, 1 \mathrm{H}), 4.22-4.12$ (m, $4 \mathrm{H}), 4.09$ (ddd, $J=10.7$, 10.7, $5.9 \mathrm{~Hz}, 1 \mathrm{H}), 4.05-4.02(\mathrm{~m}, 2 \mathrm{H}), 3.86$ (br s, $1 \mathrm{H}), 3.81$ (d, $J=5.7 \mathrm{~Hz}, 1 \mathrm{H}), 3.73$ (dd, J = 13.3, 2.9 Hz, $1 \mathrm{H}), 3.58$ (dd, $J=14.5,4.4 \mathrm{~Hz}, 1 \mathrm{H}), 3.19$ (dd, $J=13.2,12.7 \mathrm{~Hz}, 1 \mathrm{H}), 3.08-2.98$ (m, $1 \mathrm{H}), 2.50-$ $2.39(\mathrm{~m}, 2 \mathrm{H}), 2.26(\mathrm{~d}, J=14.5 \mathrm{~Hz}, 1 \mathrm{H}), 2.24(\mathrm{dd}, J=14.9,6.1 \mathrm{~Hz}, 1 \mathrm{H}), 2.09$ (s, $3 \mathrm{H}), 2.13-1.92$ (m, $16 \mathrm{H}), 2.04(\mathrm{~s}, 3 \mathrm{H}), 1.81(\mathrm{dd}, J=13.4,4.2 \mathrm{~Hz}, 1 \mathrm{H}), 1.72-1.62(\mathrm{~m}, 3 \mathrm{H}), 1.52-1.34(\mathrm{~m}, 6 \mathrm{H}), 1.08(\mathrm{~d}, J$ $=7.0 \mathrm{~Hz}, 3 \mathrm{H}), 1.00-0.92(\mathrm{~m}, 4 \mathrm{H}), 0.89(\mathrm{~d}, J=6.1 \mathrm{~Hz}, 3 \mathrm{H}), 0.86(\mathrm{~d}, J=6.6 \mathrm{~Hz}, 3 \mathrm{H}), 0.83(\mathrm{~d}, J=6.6$ $\mathrm{Hz}, 3 \mathrm{H}), 0.80(\mathrm{~d}, J=6.1 \mathrm{~Hz}, 3 \mathrm{H}), 0.78(\mathrm{~d}, J=6.6 \mathrm{~Hz}, 3 \mathrm{H}), 0.01(\mathrm{~s}, 9 \mathrm{H}) ;{ }^{13} \mathrm{C} \mathrm{NMR}(150 \mathrm{MHz}$, $\left.\mathrm{CDCl}_{3}\right): \delta=212.9,172.1,171.4,157.1,142.0,132.1,131.8,130.1,129.5,117.3,112.3,105.2,97.9$ $97.7,81.9,79.8,78.9,77.9,77.3,76.8,74.3,73.6,69.7,64.8,63.8,49.8,46.0,42.3,41.2,39.7,39.3$, $37.6,36.8,36.7,36.5,35.1,34.4,32.4,32.3,32.1,31.3,31.0,30.7,29.7,29.0,24.8,24.2,23.8,23.7$, 22.2, 22.0, 22.0, 20.3, 19.7, 18.6, 17.6, 17.5, 16.6, -0.4; HRMS (MALDI-FTMS) for $\mathrm{C}_{57} \mathrm{H}_{89} \mathrm{NO}_{15} \mathrm{SiNa}^{+}$ $\left[\mathrm{M}+\mathrm{Na}^{+}\right]$calcd 1078.5893, found 1078.5887.

FGHI Diastereomeric Des-iodo Product 36. FGHI diastereomeric octacycle 34 (21 mg, 0.018 mmol, 1.0 equiv) was dissolved in a mixture of $n-\mathrm{Bu}_{3} \mathrm{SnH}$ :toluene $(1: 2,1.5 \mathrm{~mL})$ and cooled to $0{ }^{\circ} \mathrm{C}$. $\mathrm{Et}_{3} \mathrm{~B}(1.0$

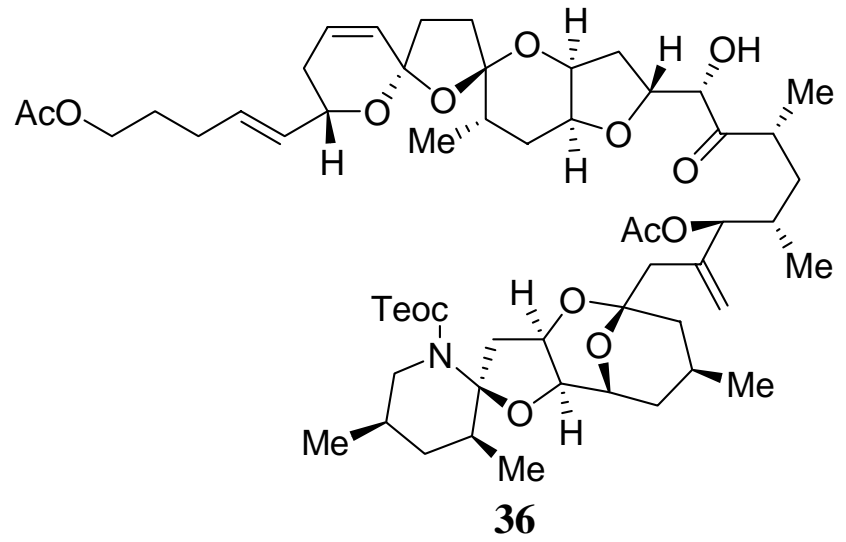
$M$ in hexanes, 1 drop) was added and the reaction mixture was stirred for $5 \mathrm{~min}$. The reaction mixture was then quenched with $\mathrm{H}_{2} \mathrm{O}(2 \mathrm{~mL})$. The aqueous layer was extracted with EtOAc $(3 \times 5 \mathrm{~mL})$ and the combined organic layers were washed with saturated aqueous $\mathrm{NaCl}(10 \mathrm{~mL})$, dried over $\mathrm{Na}_{2} \mathrm{SO}_{4}$, and concentrated. The crude product was purified by flash column chromatography (silica gel, hexanes:EtOAc 4:1) to afford FGHI diastereomeric des-iodo 
product 36 (16 mg, $0.017 \mathrm{mmol}, 94 \%)$ as a colorless oil. 36: $R_{\mathrm{f}}=0.30$ (silica gel, EtOAc:hexanes 1:2); $[\alpha]_{\mathrm{D}}=+3.0\left(c=0.60, \mathrm{CHCl}_{3}\right) ; \mathrm{IR}(\mathrm{film}) v_{\max }=2955,2908,2362,1738,1690,1458,1390,1243,1132$ $\mathrm{cm}^{-1} ;{ }^{1} \mathrm{H} \mathrm{NMR}\left(600 \mathrm{MHz}, \mathrm{CDCl}_{3}\right): \delta=5.97(\mathrm{dd}, J=7.7,5.9 \mathrm{~Hz}, 1 \mathrm{H}), 5.70(\mathrm{~d}, J=10.1 \mathrm{~Hz}, 1 \mathrm{H}), 5.66$ $(\mathrm{dd}, J=14.7,7.2 \mathrm{~Hz}, 1 \mathrm{H}), 5.51(\mathrm{dd}, J=15.3,6.1 \mathrm{~Hz}, 1 \mathrm{H}), 5.25(\mathrm{~d}, J=2.2 \mathrm{~Hz}, 1 \mathrm{H}), 5.11(\mathrm{~s}, 1 \mathrm{H}), 5.01$ (s, $1 \mathrm{H}), 4.81(\mathrm{dd}, J=7.7,7.0 \mathrm{~Hz}, 1 \mathrm{H}), 4.74$ (br s, $1 \mathrm{H}), 4.40$ (dd, $J=5.5,5.0 \mathrm{~Hz}, 1 \mathrm{H}), 4.29$ (d, $J=3.5$ Hz, $1 \mathrm{H}), 4.20-4.12$ (m, $3 \mathrm{H}), 4.10(\mathrm{dd}, J=11.2,5.9 \mathrm{~Hz}, 1 \mathrm{H}), 4.07-4.01$ (m, $2 \mathrm{H}), 3.90-3.70$ (m, 5 H), $3.20(\mathrm{t}, J=12.5 \mathrm{~Hz}, 1 \mathrm{H}), 3.06-2.98(\mathrm{~m}, 1 \mathrm{H}), 2.38(\mathrm{ddd}, J=12.9,9.6,3.5 \mathrm{~Hz}, 1 \mathrm{H}), 2.30(\mathrm{~d}, J=14.5$ Hz, $1 \mathrm{H}), 2.22$ (d, J=14.5 Hz, $1 \mathrm{H}), 2.14$ (dd, J=14.9, $5.3 \mathrm{~Hz}, 1 \mathrm{H}), 2.08$ (s, $3 \mathrm{H}), 2.04$ (s, 3 H), $2.04-$ $1.88(\mathrm{~m}, 15 \mathrm{H}), 1.82(\mathrm{dd}, J=13.6,3.9 \mathrm{~Hz}, 1 \mathrm{H}), 1.72-1.62(\mathrm{~m}, 5 \mathrm{H}), 1.42-1.30(\mathrm{~m}, 5 \mathrm{H}), 1.08(\mathrm{~d}, J=7.0$ $\mathrm{Hz}, 3 \mathrm{H}), 1.04-0.96(\mathrm{~m}, 4 \mathrm{H}), 0.90(\mathrm{~d}, J=5.7 \mathrm{~Hz}, 3 \mathrm{H}), 0.86(\mathrm{~d}, J=6.1 \mathrm{~Hz}, 3 \mathrm{H}), 0.85(\mathrm{~d}, J=6.6 \mathrm{~Hz}, 3$ $\mathrm{H}), 0.81(\mathrm{~d}, J=6.6 \mathrm{~Hz}, 3 \mathrm{H}), 0.80(\mathrm{~d}, J=6.1 \mathrm{~Hz}, 3 \mathrm{H}), 0.01(\mathrm{~s}, 9 \mathrm{H}) ;{ }^{13} \mathrm{C} \mathrm{NMR}\left(150 \mathrm{MHz}, \mathrm{CDCl}_{3}\right): \delta=$ 213.2, 172.1, 171.2, 156.9, 142.7, 132.1, 131.8, 130.2, 129.5, 116.2, 112.4, 105.2, 98.0, 97.2, 81.2, 79.4, 79.1, 77.9, 77.2, 76.9, 74.6, 72.3, 69.8, 64.8, 63.7, 49.4, 46.3, 41.7, 40.1, 39.4, 37.6, 36.9, 36.7, 35.8, $34.9,34.2,32.6,32.4,32.0,31.0,30.7,29.7,29.0,25.6,24.3,24.0,22.1,22.0,22.0,19.7,18.8,17.9$, 17.5, 16.6, -0.5; HRMS (MALDI-FTMS) for $\mathrm{C}_{57} \mathrm{H}_{89} \mathrm{NO}_{15} \mathrm{SiNa}^{+}\left[\mathrm{M}+\mathrm{Na}^{+}\right]$calcd 1078.5893, found 1078.5868 .

Primary Hydroxyl 39. Des-iodo product 35 (16 mg, $0.014 \mathrm{mmol}, 1.0$ equiv) was dissolved in $\mathrm{CH}_{2} \mathrm{Cl}_{2}$ ( $3 \mathrm{~mL}$ ) and cooled to $-78{ }^{\circ} \mathrm{C}$. 2,6-Lutidine ( $0.050 \mathrm{~mL}, 0.28 \mathrm{mmol}, 20$ equiv) was added, followed by

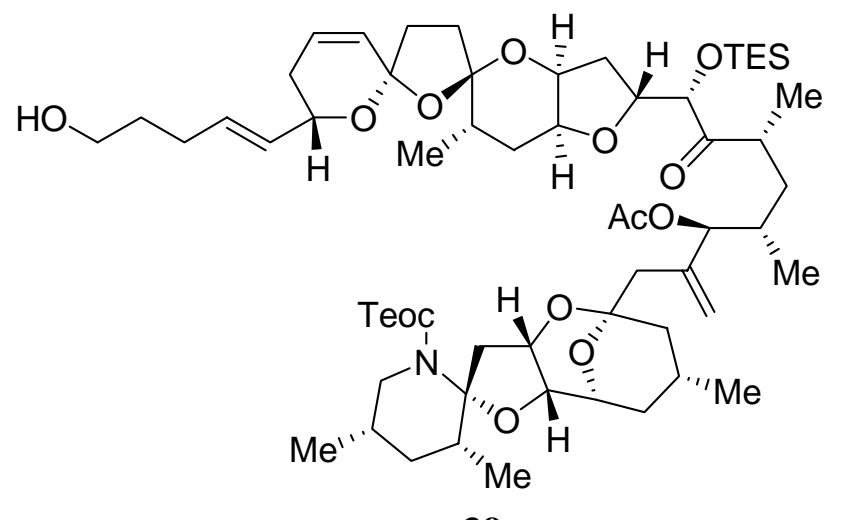

39
TESOTf (0.050 mL, $0.14 \mathrm{mmol}, 10$ equiv). After being stirred for $10 \mathrm{~min}$ at $0{ }^{\circ} \mathrm{C}$ the reaction mixture was then quenched by the addition of saturated aqueous $\mathrm{NH}_{4} \mathrm{Cl}(5 \mathrm{~mL})$ and the two layers were separated. The aqueous layer was extracted with $\mathrm{CH}_{2} \mathrm{Cl}_{2}(3 \times 10 \mathrm{~mL})$ and the combined organic layers were washed with $\mathrm{H}_{2} \mathrm{O}(10 \mathrm{~mL})$ and saturated aqueous $\mathrm{NaCl}(10 \mathrm{~mL})$, and then dried over $\mathrm{Na}_{2} \mathrm{SO}_{4}$, 
filtered, and concentrated. The crude material was then dissolved in $\mathrm{MeOH}(2.5 \mathrm{~mL})$ at $25{ }^{\circ} \mathrm{C} . \mathrm{K}_{2} \mathrm{CO}_{3}$ (13 mg, 0.009 mmol, 1.0 equiv) was added and the reaction mixture was stirred at $25^{\circ} \mathrm{C}$ for $2 \mathrm{~h}$. The reaction mixture was diluted with $\mathrm{H}_{2} \mathrm{O}(5 \mathrm{~mL})$ and extracted with EtOAc $(3 \times 5 \mathrm{~mL})$. The combined organic layers were washed with brine $(10 \mathrm{~mL})$, dried over $\mathrm{Na}_{2} \mathrm{SO}_{4}$, and concentrated. The crude product was purified by flash column chromatography (silica gel, hexanes:EtOAc 2:1) to afford primary hydroxyl 39 (12 mg, $0.011 \mathrm{mmol}, 76 \%$ over two steps) as a colorless oil. 39: $R_{\mathrm{f}}=0.22$ (silica gel, EtOAc:hexanes 3:2); $[\alpha]_{\mathrm{D}}=+7.5\left(c=0.80, \mathrm{CHCl}_{3}\right)$; IR (film) $v_{\max }=3483,2955,1731,1694,1458$, 1361, 1172, $1132 \mathrm{~cm}^{-1} ;{ }^{1} \mathrm{H} \mathrm{NMR}\left(600 \mathrm{MHz}, \mathrm{CDCl}_{3}\right): \delta=5.97(\mathrm{ddd}, J=9.7,5.5,2.0 \mathrm{~Hz}, 1 \mathrm{H}), 5.73-$ $5.66(\mathrm{~m}, 2 \mathrm{H}), 5.52(\mathrm{dd}, J=15.4,6.2 \mathrm{~Hz}, 1 \mathrm{H}), 5.27(\mathrm{~s}, 1 \mathrm{H}), 5.11(\mathrm{~s}, 1 \mathrm{H}), 5.00(\mathrm{~d}, J=7.0 \mathrm{~Hz}, 1 \mathrm{H})$, 4.76-4.69 (m, $1 \mathrm{H}), 4.68-4.63(\mathrm{~m}, 2 \mathrm{H}), 4.41(\mathrm{ddd}, J=10.4,5.6,4.5 \mathrm{~Hz}, 1 \mathrm{H}), 4.28(\mathrm{~d}, J=4.8 \mathrm{~Hz}, 1 \mathrm{H})$, $4.18(\mathrm{~d}, J=4.4 \mathrm{~Hz}, 1 \mathrm{H}), 4.16-4.09(\mathrm{~m}, 2 \mathrm{H}), 4.01(\mathrm{dt}, J=10.8,6.2 \mathrm{~Hz}, 1 \mathrm{H}), 3.88-3.78(\mathrm{~m}, 1 \mathrm{H}), 3.77-$ $3.76(\mathrm{~m}, 1 \mathrm{H}), 3.72(\mathrm{~d}, J=4.8 \mathrm{~Hz}, 1 \mathrm{H}), 3.63(\mathrm{t}, J=6.4 \mathrm{~Hz}, 2 \mathrm{H}), 3.20(\mathrm{t}, J=12.3,1 \mathrm{H}), 3.08-3.00(\mathrm{~m}, 1$ H), 2.18-2.10 (m, 6 H), 2.10-1.93 (m, $12 \mathrm{H}), 2.07$ (s, $3 \mathrm{H}), 1.69-1.52(\mathrm{~m}, 8 \mathrm{H}), 1.04(\mathrm{~d}, J=7.0 \mathrm{~Hz}, 3$ H), $0.94(\mathrm{t}, J=7.9 \mathrm{~Hz}, 9 \mathrm{H}), 0.88(\mathrm{~d}, J=5.5 \mathrm{~Hz}, 3 \mathrm{H}), 0.88(\mathrm{~d}, J=7.0 \mathrm{~Hz}, 3 \mathrm{H}), 0.81(\mathrm{~d}, J=6.6 \mathrm{~Hz}, 3$ H), $0.81(\mathrm{~d}, J=5.9 \mathrm{~Hz}, 3 \mathrm{H}), 0.78(\mathrm{~d}, J=6.6 \mathrm{~Hz}, 3 \mathrm{H}), 0.61(\mathrm{q}, J=7.9 \mathrm{~Hz}, 6 \mathrm{H}), 0.01(\mathrm{~s}, 9 \mathrm{H}) ;{ }^{13} \mathrm{C} \mathrm{NMR}$ $\left(150 \mathrm{MHz}, \mathrm{CDCl}_{3}\right): \delta=213.4,171.3,156.9,141.1,132.9,131.5,130.2,129.4,119.4,112.4,105.1,98.0$, $97.1,83.0,80.4,80.3,79.1,77.1,77.0,74.5,72.2,69.8,63.6,63.3,49.6,44.4,44.3,40.5,40.2,40.1$, $37.5,33.4,32.4,32.0,31.3,31.0,30.7,29.7,25.6,24.3,24.1,22.4,19.7,19.7,18.8,18.0,17.5,16.6,7.9$, 5.9, -0.5; HRMS (MALDI-FTMS) for $\mathrm{C}_{61} \mathrm{H}_{101} \mathrm{NO}_{14} \mathrm{Si}_{2} \mathrm{Na}^{+}\left[\mathrm{M}+\mathrm{Na}^{+}\right]$calcd 1150.6652, found 1150.6636.

FGHI Diastereomeric Primary Hydroxyl 40. FGHI diastereomeric des-iodo product 36 (16 mg, 0.014 mmol, 1.0 equiv) was dissolved in $\mathrm{CH}_{2} \mathrm{Cl}_{2}(3 \mathrm{~mL})$ and cooled to $-78{ }^{\circ} \mathrm{C} .2,6$-Lutidine $(0.050 \mathrm{~mL}, 0.28$ mmol, 20 equiv) was added, followed by TESOTf $(0.050 \mathrm{~mL}, 0.14 \mathrm{mmol}, 10$ equiv $)$. After being stirred for $10 \mathrm{~min}$ at $0{ }^{\circ} \mathrm{C}$ the reaction mixture was quenched by the addition of saturated aqueous $\mathrm{NH}_{4} \mathrm{Cl}(5$ $\mathrm{mL})$ and the two layers were separated. The aqueous layer was extracted with $\mathrm{CH}_{2} \mathrm{Cl}_{2}(3 \times 10 \mathrm{~mL})$ and the combined organic layers were washed with $\mathrm{H}_{2} \mathrm{O}(10 \mathrm{~mL})$ and saturated aqueous $\mathrm{NaCl}(10 \mathrm{~mL})$, dried 
over $\mathrm{Na}_{2} \mathrm{SO}_{4}$, filtered, and concentrated. The crude material was dissolved in $\mathrm{MeOH}(2.5 \mathrm{~mL}) . \mathrm{K}_{2} \mathrm{CO}_{3}$

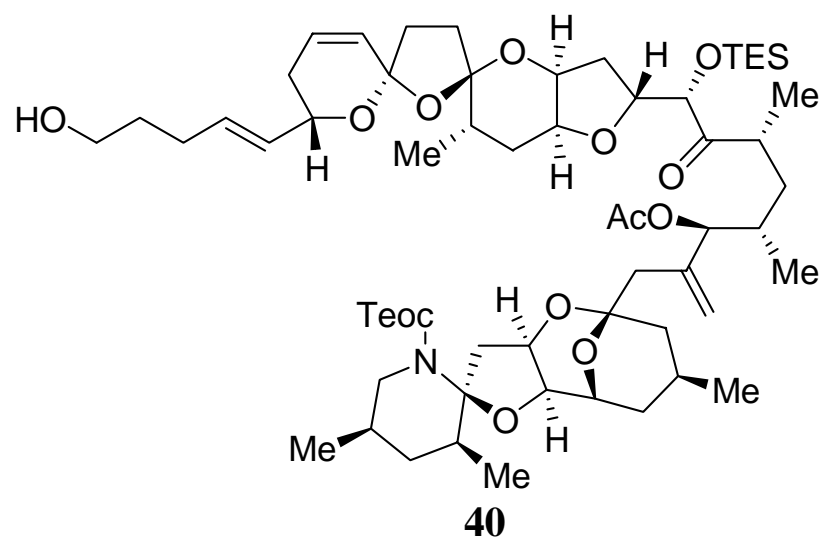
(13 mg, $0.009 \mathrm{mmol}, 1.0$ equiv) was added and the reaction mixture was stirred at $25{ }^{\circ} \mathrm{C}$ for $2 \mathrm{~h}$. The reaction mixture was diluted with $\mathrm{H}_{2} \mathrm{O}(5 \mathrm{~mL})$ and extrated with EtOAc $(3 \times 5 \mathrm{~mL})$. The combined organic layers were washed with brine $(10 \mathrm{~mL})$, dried over $\mathrm{Na}_{2} \mathrm{SO}_{4}$, and concentrated. The crude product was purified by flash column chromatography (silica gel, hexanes:EtOAc 2:1) to afford primary hydroxyl

40 (11 mg, $0.0098 \mathrm{mmol}, 70 \%$ over two steps) as a colorless oil. 40: $R_{\mathrm{f}}=0.25$ (silica gel, EtOAc:hexanes 3:2); $[\alpha]_{\mathrm{D}}=+10.3\left(c=1.20, \mathrm{CHCl}_{3}\right)$; IR (film) $v_{\max }=3472,2955,1736,1690,1458$, 1390, 1244, $1172 \mathrm{~cm}^{-1} ;{ }^{1} \mathrm{H}$ NMR (600 MHz, $\left.\mathrm{CDCl}_{3}\right): \delta=6.02-5.94(\mathrm{~m}, 1 \mathrm{H}), 5.74-5.66(\mathrm{~m}, 2 \mathrm{H}), 5.52$ (dd, $J=14.3,5.9 \mathrm{~Hz}, 1 \mathrm{H}), 5.18$ (d, $J=4.8 \mathrm{~Hz}, 1 \mathrm{H}), 5.14$ (s, $1 \mathrm{H}), 5.04(\mathrm{~s}, 1 \mathrm{H}), 4.76-4.71(\mathrm{~m}, 1 \mathrm{H})$, 4.69-4.63 (m, $1 \mathrm{H}), 4.44-4.38(\mathrm{~m}, 1 \mathrm{H}), 4.29$ (d, J = 4.4 Hz, $1 \mathrm{H}), 4.21-4.03$ (m, $4 \mathrm{H}), 3.84-3.74$ (m, 4 H), $3.63(\mathrm{~d}, J=6.4 \mathrm{~Hz}, 2 \mathrm{H}), 3.21(\mathrm{dd}, J=13.2,12.7 \mathrm{~Hz}, 1 \mathrm{H}), 3.08-2.98(\mathrm{~m}, 1 \mathrm{H}), 2.22(\mathrm{~d}, J=14.2 \mathrm{~Hz}$, $1 \mathrm{H}), 2.17-1.92(\mathrm{~m}, 18 \mathrm{H}), 2.06(\mathrm{~s}, 3 \mathrm{H}), 1.87-1.80(\mathrm{~m}, 1 \mathrm{H}), 1.78$ (dd, J=13.4, $5.0 \mathrm{~Hz}, 1 \mathrm{H}), 1.68-1.52$ (m, $6 \mathrm{H}), 1.44-1.30(\mathrm{~m}, 5 \mathrm{H}), 1.04(\mathrm{~d}, J=7.0 \mathrm{~Hz}, 3 \mathrm{H}), 0.93$ (t, $J=7.9 \mathrm{~Hz}, 9 \mathrm{H}), 0.89$ (d, J=6.1 Hz, 3 H), $0.87(\mathrm{~d}, J=7.0 \mathrm{~Hz}, 3 \mathrm{H}), 0.83(\mathrm{~d}, J=6.6 \mathrm{~Hz}, 3 \mathrm{H}), 0.81(\mathrm{~d}, J=6.6 \mathrm{~Hz}, 3 \mathrm{H}), 0.61(\mathrm{~d}, J=7.7 \mathrm{~Hz}, 3$ $\mathrm{H}), 0.02(\mathrm{~s}, 9 \mathrm{H}) ;{ }^{13} \mathrm{C} \mathrm{NMR}\left(150 \mathrm{MHz}, \mathrm{CDCl}_{3}\right): \delta=213.2,171.2,156.9,142.6,132.9,131.5,130.2$, $129.4,117.0,112.4,105.1,97.9,97.3,81.4,80.5,80.3,79.1,77.1,77.1,74.6,72.2,69.8,63.6,63.3$, $49.5,46.1,41.9,40.6,40.1,37.5,36.9,36.7,35.8,35.0,34.9,33.5,33.0,32.5,31.8,31.3,29.7,25.6$, $24.3,24.0,22.2,19.7,19.6,18.9,18.1,17.5,16.6,15.1,-0.5$; HRMS (MALDI-FTMS) for $\mathrm{C}_{61} \mathrm{H}_{101} \mathrm{NO}_{14} \mathrm{Si}_{2} \mathrm{Na}^{+}\left[\mathrm{M}+\mathrm{Na}^{+}\right]$calcd 1150.6652 , found 1150.6670 .

Free amine 45 . To a solution of oxalyl chloride $\left(2 \mu \mathrm{L}, 0.12 \mathrm{mmol}, 10.0\right.$ equiv) in $\mathrm{CH}_{2} \mathrm{Cl}_{2}(1 \mathrm{~mL})$ at -78 ${ }^{\circ} \mathrm{C}$ was added DMSO (15 $\mu \mathrm{L}, 0.24 \mathrm{mmol}, 20.0$ equiv). The solution was allowed to stir for $20 \mathrm{~min}$ at $78{ }^{\circ} \mathrm{C}$ and then a solution of primary hydroxyl 39 (13.5 mg, $0.012 \mathrm{mmol}, 1.0$ equiv) in $\mathrm{CH}_{2} \mathrm{Cl}_{2}(0.5 \mathrm{~mL})$ 
was added and the resulting mixture was allowed to stir at $-78^{\circ} \mathrm{C}$ for $1 \mathrm{~h} . \mathrm{Et}_{3} \mathrm{~N}(0.086 \mathrm{~mL}, 0.60 \mathrm{mmol}$,

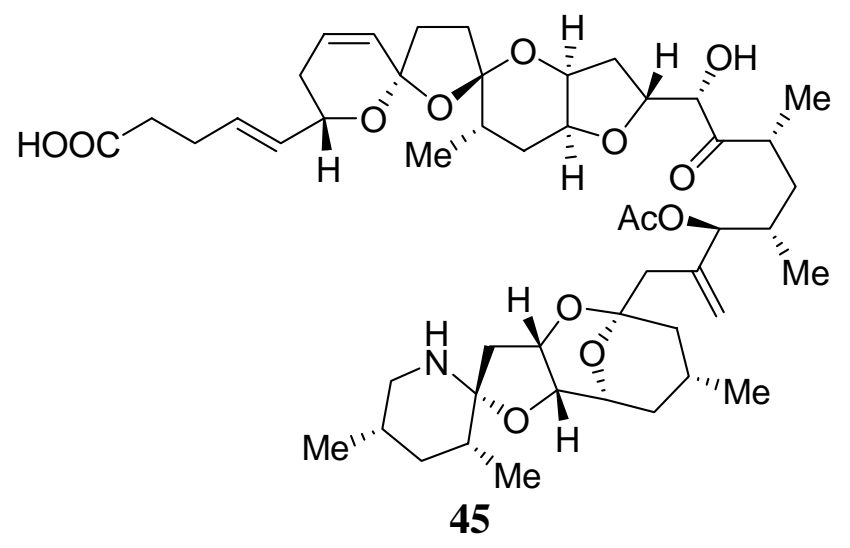

50 equiv) was then added and the reaction mixture was allowed to warm to $0{ }^{\circ} \mathrm{C}$ over $1 \mathrm{~h}$. The reaction mixture was then quenched with saturated aqueous $\mathrm{NaCl}(1 \mathrm{~mL})$ and then extracted with $\mathrm{CH}_{2} \mathrm{Cl}_{2}(3 \times 1$ $\mathrm{mL})$. The combined organic layers were dried $\left(\mathrm{Na}_{2} \mathrm{SO}_{4}\right)$ and concentrated, and the obtained aldehyde 41 was used in the next step without further purification. Aldehyde 41 (13.5 $\mathrm{mg}, 0.012 \mathrm{mmol}, 1.0$ equiv) was dissolved in $t-\mathrm{BuOH}: \mathrm{H}_{2} \mathrm{O}(4: 1,1 \mathrm{~mL})$ at $25^{\circ} \mathrm{C}$, and to the solution was added $\mathrm{NaClO}_{2}(11$ mg, 0.12 mmol, 10.0 equiv), $\mathrm{NaH}_{2} \mathrm{PO}_{4}$ (18 mg, $0.12 \mathrm{mmol}, 10.0$ equiv), and 2-methyl-2-butene (0.2 $\mathrm{mL}$ ). The reaction mixture was allowed to stir at $25^{\circ} \mathrm{C}$ for $1.5 \mathrm{~h}$, then quenched with a $\mathrm{pH} 7 \mathrm{buffered}$ saline solution $(3 \mathrm{~mL})$. The resulting biphasic mixture was then extracted with EtOAc $(3 \times 2 \mathrm{~mL})$ and the combined organic layers were dried $\left(\mathrm{Na}_{2} \mathrm{SO}_{4}\right)$ and concentrated to afford crude carboxylic acid 43 which was used in the next step without further purification. Carboxylic acid 43 (13 mg, 0.012 mmol, 1.0 equiv) was dissolved in THF $(3 \mathrm{~mL})$ and cooled to $0{ }^{\circ} \mathrm{C}$. TBAF $(0.060 \mathrm{~mL}, 1.0 \mathrm{M}$ in THF, 0.060 mmol) was added and the mixture was stirred for $2 \mathrm{~h}$ at $25^{\circ} \mathrm{C}$. The reaction mixture was quenched with saturated aqueous $\mathrm{NH}_{4} \mathrm{Cl}(5 \mathrm{~mL})$ and extracted with EtOAc $(3 \times 10 \mathrm{~mL})$. The combined organic extracts were washed with saturated aqueous $\mathrm{NaCl}(10 \mathrm{~mL})$, dried over $\mathrm{Na}_{2} \mathrm{SO}_{4}$, filtered, and concentrated. The crude product was purified by preparative TLC (silica gel, $\mathrm{CHCl}_{3}: \mathrm{MeOH}: \mathrm{H}_{2} \mathrm{O}$ 10:3:1) to afford free amine 45 (7.4 mg, $0.0084 \mathrm{mmol}, 70 \%$ over three steps) as a colorless oil. 45: $R_{\mathrm{f}}=0.35$ (silica gel, $\left.\mathrm{CHCl}_{3}: \mathrm{MeOH}: \mathrm{H}_{2} \mathrm{O} 20: 3: 1\right) ;[\alpha]_{\mathrm{D}}=-22.4\left(c=0.38, \mathrm{CHCl}_{3}\right) ; \mathrm{IR}($ film $) v_{\max }=3333,2957,1731,1574$, $1425,1242,1138 \mathrm{~cm}^{-1} ;{ }^{1} \mathrm{H} \mathrm{NMR}\left(600 \mathrm{MHz}, \mathrm{CD}_{3} \mathrm{OD}\right): \delta=6.00(\mathrm{ddd}, J=9.6,5.5,1.8 \mathrm{~Hz}, 1 \mathrm{H}), 5.77-$ $5.73(\mathrm{~m}, 1 \mathrm{H}), 5.66(\mathrm{dt}, J=9.9,1.9 \mathrm{~Hz}, 1 \mathrm{H}), 5.50(\mathrm{dd}, J=15.3,6.1 \mathrm{~Hz}, 1 \mathrm{H}), 5.39$ (br s, $1 \mathrm{H}), 5.10(\mathrm{~s}, 1$ H), $5.05(\mathrm{~s}, 1 \mathrm{H}), 4.80-4.79(\mathrm{~m}, 2 \mathrm{H}), 4.33(\mathrm{dd}, J=13.8,6.8 \mathrm{~Hz}, 1 \mathrm{H}), 4.28($ br s, $1 \mathrm{H}), 4.24(\mathrm{~d}, J=4.4$ Hz, $1 \mathrm{H}), 4.14(\mathrm{~d}, J=2.2 \mathrm{~Hz}, 1 \mathrm{H}), 3.87$ (br s, $1 \mathrm{H}), 3.70$ (d, J=3.5 Hz, 1H), 3.19-3.16 (m, $1 \mathrm{H}), 2.66$ (t, $J=11.4 \mathrm{~Hz}, 1 \mathrm{H}), 2.56(\mathrm{dd}, J=11.2,4.2 \mathrm{~Hz}, 1 \mathrm{H}), 2.40-2.28(\mathrm{~m}, 5 \mathrm{H}), 2.24-2.18$ (m, $3 \mathrm{H}), 2.09$ (s, 3 
H), 2.10-1.98 (m, 7 H), 1.98-1.87 (m, 4 H), 1.81-1.71 (m, 3 H), 1.70-1.62 (m, 2 H), 1.55-1.45 (m, 3 H), $1.05(\mathrm{~d}, J=7.0 \mathrm{~Hz}, 3 \mathrm{H}), 0.97-0.90(\mathrm{~m}, 3 \mathrm{H}), 0.92(\mathrm{~d}, J=5.7 \mathrm{~Hz}, 3 \mathrm{H}), 0.91(\mathrm{~d}, J=5.7 \mathrm{~Hz}, 3 \mathrm{H})$, $0.88(\mathrm{~d}, J=6.6 \mathrm{~Hz}, 3 \mathrm{H}), 0.86(\mathrm{~d}, J=6.6 \mathrm{~Hz}, 3 \mathrm{H}), 0.85(\mathrm{~d}, J=6.6 \mathrm{~Hz}, 3 \mathrm{H}) ;{ }^{13} \mathrm{C}$ NMR $(150 \mathrm{MHz}$, $\left.\mathrm{CD}_{3} \mathrm{OD}\right): \delta=216.7,182.8,173.1,144.1,133.9,131.9,130.7,130.7,116.6,113.8,106.6,98.6,96.9$, $82.1,80.9,80.2,79.2,79.1,78.5,76.5,73.5,71.3,50.4,48.3,44.2,43.5,40.9,40.4,39.6,38.9,37.8$, $37.6,36.5,35.8,35.5,33.9,33.2,32.6,32.1,31.4,27.2,25.2,24.8,22.0,20.7,20.4,18.4,17.1,17.0$; HRMS (MALDI-FTMS) for $\mathrm{C}_{49} \mathrm{H}_{73} \mathrm{NO}_{13} \mathrm{H}^{+}\left[\mathrm{M}+\mathrm{H}^{+}\right]$calcd 884.5154, found 884.5119.

FGHI Diastereomeric Free Amine 46. To a solution of oxalyl chloride ( $2 \mu \mathrm{L}, 0.12 \mathrm{mmol}, 10.0$ equiv) in $\mathrm{CH}_{2} \mathrm{Cl}_{2}(1 \mathrm{~mL})$ at $-78{ }^{\circ} \mathrm{C}$ was added DMSO $(15 \mu \mathrm{L}, 0.24 \mathrm{mmol}, 20.0$ equiv). The solution was

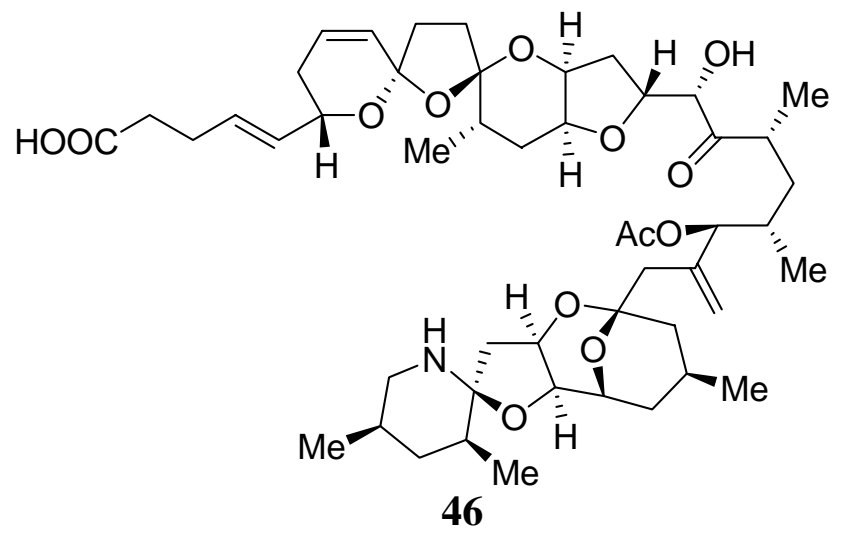
allowed to stir for $20 \mathrm{~min}$ at $-78{ }^{\circ} \mathrm{C}$, and then a solution of FGHI diastereomeric primary hydroxyl $\mathbf{4 0}$ (13.5 mg, $0.012 \mathrm{mmol}, 1.0$ equiv) in $\mathrm{CH}_{2} \mathrm{Cl}_{2}(0.5 \mathrm{~mL})$ was added. The resulting mixture was allowed to stir at $-78^{\circ} \mathrm{C}$ for $1 \mathrm{~h}$, at which time $\mathrm{Et}_{3} \mathrm{~N}(0.086 \mathrm{~mL}, 0.60$ mmol, 50 equiv) was added and the reaction mixture was allowed to warm to $0{ }^{\circ} \mathrm{C}$ over $1 \mathrm{~h}$. The reaction mixture was then quenched with saturated aqueous $\mathrm{NaCl}(1 \mathrm{~mL})$ and extracted with $\mathrm{CH}_{2} \mathrm{Cl}_{2}(3 \times 1 \mathrm{~mL})$. The combined organic extracts were dried $\left(\mathrm{Na}_{2} \mathrm{SO}_{4}\right)$ and concentrated, and the obtained FGHI diastereomeric aldehyde $\mathbf{4 2}$ was used in the next step without further purification. FGHI diastereomeric aldehyde 42 (13.5 mg, $0.012 \mathrm{mmol}, 1.0$ equiv) was dissolved in $t-\mathrm{BuOH}: \mathrm{H}_{2} \mathrm{O}(4: 1,1 \mathrm{~mL})$ at $25{ }^{\circ} \mathrm{C}$, and to the solution was added $\mathrm{NaClO}_{2}\left(11 \mathrm{mg}, 0.12 \mathrm{mmol}, 10.0\right.$ equiv), $\mathrm{NaH}_{2} \mathrm{PO}_{4}(18 \mathrm{mg}, 0.12 \mathrm{mmol}, 10.0$ equiv), and 2-methyl-2-butene $(0.2 \mathrm{~mL})$. The reaction mixture was allowed to stir at $25{ }^{\circ} \mathrm{C}$ for $1.5 \mathrm{~h}$, then quenched with a pH 7 buffered saline solution $(3 \mathrm{~mL})$, and then extracted with EtOAc $(3 \times 2 \mathrm{~mL})$. The combined organic extracts were dried $\left(\mathrm{Na}_{2} \mathrm{SO}_{4}\right)$ and concentrated to afford crude FGHI diastereomeric carboxylic acid $\mathbf{4 4}$ which was used in the next step without further purification. FGHI diastereomeric carboxylic acid 44 (13 mg, $0.012 \mathrm{mmol}, 1.0$ equiv) was dissolved in THF (3 mL) and 
cooled to $0{ }^{\circ} \mathrm{C}$. TBAF $(0.060 \mathrm{~mL}, 1.0 \mathrm{M}$ in THF, $0.060 \mathrm{mmol})$ was added and the mixture was stirred for $2 \mathrm{~h}$ at $25{ }^{\circ} \mathrm{C}$. The reaction mixture was then quenched with saturated aqueous $\mathrm{NH}_{4} \mathrm{Cl}(5 \mathrm{~mL})$ and extracted with EtOAc $(3 \times 10 \mathrm{~mL})$. The combined organic extracts were washed with saturated aqueous $\mathrm{NaCl}(10 \mathrm{~mL})$, dried over $\mathrm{Na}_{2} \mathrm{SO}_{4}$, filtered, and concentrated. The crude product was purified by preparative TLC (silica gel, $\mathrm{CHCl}_{3}: \mathrm{MeOH}: \mathrm{H}_{2} \mathrm{O}$ 10:3:1) to afford FGHI diastereomeric free amine 46 (7.7 mg, $0.0088 \mathrm{mmol}, 73 \%$ over three steps) as a colorless oil. 46: $R_{\mathrm{f}}=0.30$ (silica gel, $\left.\mathrm{CHCl}_{3}: \mathrm{MeOH}: \mathrm{H}_{2} \mathrm{O} 20: 3: 1\right) ;[\alpha]_{\mathrm{D}}=+9.50\left(c=0.60, \mathrm{CHCl}_{3}\right) ;$ IR (film) $v_{\max }=3422,2956,1734,1649$, 1562, 1403, 1320, 1240, $1132 \mathrm{~cm}^{-1} ;{ }^{1} \mathrm{H}$ NMR (600 MHz, $\left.\mathrm{CD}_{3} \mathrm{OD}\right): \delta=6.00$ (ddd, $J=9.9,3.7,3.2 \mathrm{~Hz}, 1$ H), 5.76-5.73 (m, $1 \mathrm{H}), 5.66(\mathrm{dt}, J=10.1,2.0 \mathrm{~Hz}, 1 \mathrm{H}), 5.50(\mathrm{dd}, J=15.8,6.1 \mathrm{~Hz}, 1 \mathrm{H}), 5.33(\mathrm{~d}, J=4.8$ Hz, $1 \mathrm{H}), 5.12$ (s, $1 \mathrm{H}), 5.04(\mathrm{~s}, 1 \mathrm{H}), 4.79-4.75(\mathrm{~m}, 2 \mathrm{H}), 4.33$ (dd, J=13.8, 7.7 Hz, $1 \mathrm{H}), 4.27-4.19$ (m, $2 \mathrm{H}), 3.87(\mathrm{~m}, 1 \mathrm{H}), 3.72(\mathrm{~d}, J=3.1 \mathrm{~Hz}, 1 \mathrm{H}), 3.18-3.13(\mathrm{~m}, 1 \mathrm{H}), 2.69-2.62(\mathrm{~m}, 2 \mathrm{H}), 2.39-2.26(\mathrm{~m}, 5$ H), 2.24-2.18 (m, 3 H), 2.08 (s, 3 H), 2.10-1.98 (m, 7 H), 1.98-1.84 (m, 4 H), 1.81-1.66 (m, 4 H), $1.70-$ $1.62(\mathrm{~m}, 1 \mathrm{H}), 1.55-1.35(\mathrm{~m}, 4 \mathrm{H}), 1.07$ (d, J=7.0 Hz, $3 \mathrm{H}), 1.05-0.90(\mathrm{~m}, 3 \mathrm{H}), 0.91(\mathrm{~d}, J=6.1 \mathrm{~Hz}, 3$ H), $0.90(\mathrm{~d}, J=6.6 \mathrm{~Hz}, 3 \mathrm{H}), 0.87(\mathrm{~d}, J=7.0 \mathrm{~Hz}, 3 \mathrm{H}), 0.87$ (d, $J=6.6 \mathrm{~Hz}, 3 \mathrm{H}), 0.84$ (d, J=6.6 Hz, 3 $\mathrm{H}) ;{ }^{13} \mathrm{C}$ NMR (150 MHz, $\left.\mathrm{CD}_{3} \mathrm{OD}\right): \delta=215.7,182.3,172.7,143.9,133.5,131.5,130.4,130.2,116.5$, 113.4, 106.1, 98.3, 96.4, 81.7, 80.4, 79.9, 78.8, 78.3, 78.0, 76.1, 73.1, 70.8, 47.6, 45.5, 43.7, 42.0, 40.5, $40.0,39.2,38.4,37.3,37.3,35.9,35.3,35.2,33.1,32.8,32.2,32.1,31.8,31.0,26.8,24.8,24.2,21.4$, 20.4, 20.0, 17.8, 16.6; HRMS (MALDI-FTMS) for $\mathrm{C}_{49} \mathrm{H}_{73} \mathrm{NO}_{13} \mathrm{H}^{+}\left[\mathrm{M}+\mathrm{H}^{+}\right]$calcd 884.5154, found 884.5119 .

Originally Proposed Azaspiracid-1 Structure 1a. To a solution of free amine 45 (4.0 mg, 0.0045 mmol) in $\mathrm{MeOH}: \mathrm{H}_{2} \mathrm{O}(5: 1,1 \mathrm{~mL})$ at $25^{\circ} \mathrm{C}$ was added a solution of $\mathrm{LiOH}(45 \mu \mathrm{L}, 0.045 \mathrm{mmol}, 1.0 \mathrm{M}$ in $\mathrm{H}_{2} \mathrm{O}, 10$ equiv). The reaction mixture was allowed to stir for $16 \mathrm{~h}$ at $25^{\circ} \mathrm{C}$, at which time the starting material was consumed (TLC). The reaction mixture was then directly purified by preparative TLC (silica gel, $\mathrm{CH}_{3} \mathrm{Cl}: \mathrm{CH}_{3} \mathrm{OH}: \mathrm{H}_{2} \mathrm{O}$ 20:3:1) to afford what is believed to be the originally proposed azaspiracid-1 structure 1a as a colorless oil (1.7 $\mathrm{mg}, 0.0020 \mathrm{mmol}, 45 \%)$ existing as an inseparable mixture of compounds. Further purification by HPLC using the conditions described in the isolation of 
azaspiracid- $1^{1}$ failed to yield a pure sample of 1a. Originally proposed azaspiracid-1 structure 1a was also synthesized by first dissolving carboxylic acid $76(5.0 \mathrm{mg}, 0.0042 \mathrm{mmol})$ in $\mathrm{THF}(1 \mathrm{~mL})$ at $25^{\circ} \mathrm{C}$. To the solution was added TBAF $(21 \mu \mathrm{L}, 0.021 \mathrm{mmol}, 1.0 \mathrm{M}$ in THF, 5.0 equiv) and the reaction

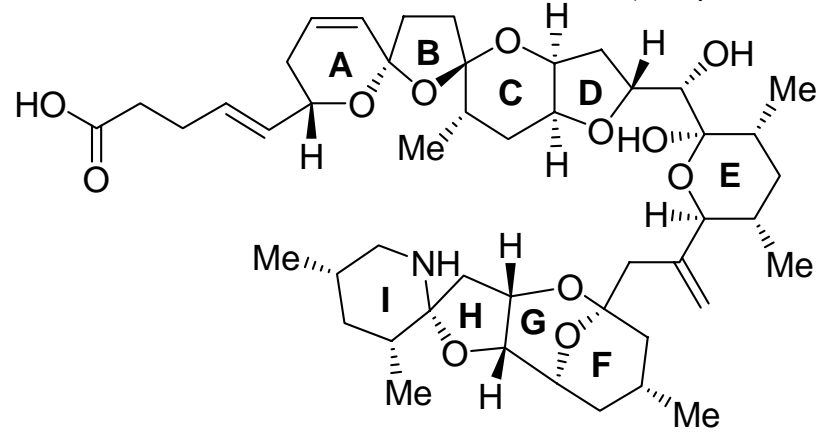

1a mixture was allowed to stir at $25^{\circ} \mathrm{C}$ for $15 \mathrm{~h}$ at which time the reaction was finished (TLC). The reaction mixture was then directly purified by preparative TLC (silica gel, $\mathrm{CH}_{3} \mathrm{Cl}: \mathrm{CH}_{3} \mathrm{OH}: \mathrm{H}_{2} \mathrm{O} 20: 3: 1$ ) to afford what is believed to be originally proposed azaspiracid-1 1a as a colorless oil (1.6 mg, $0.0019 \mathrm{mmol}, 45 \%)$ existing as an inseparable mixture of compounds. Further purification by HPLC using the conditions described in the isolation of azaspiracid- $1^{1}$ failed to yield a pure sample. 1a: $R_{\mathrm{f}}=0.42$ (silica gel, $\mathrm{CH}_{3} \mathrm{Cl}: \mathrm{CH}_{3} \mathrm{OH}: \mathrm{H}_{2} \mathrm{O}$ 20:3:1); HRMS (MALDI-FTMS) for $\mathrm{C}_{47} \mathrm{H}_{71} \mathrm{NO}_{12} \mathrm{H}^{+}\left[\mathrm{M}+\mathrm{H}^{+}\right]$calcd 842.5049, found 842.5049 .

FGHI Diastereomeric Originally Proposed Azaspiracid-1 Structure 1b. To a solution of free amine $45(4.0 \mathrm{mg}, 0.0045 \mathrm{mmol})$ in $\mathrm{MeOH}: \mathrm{H}_{2} \mathrm{O}(5: 1,1 \mathrm{~mL})$ at $25^{\circ} \mathrm{C}$ was added a solution of $\mathrm{LiOH}(45 \mu \mathrm{L}$,

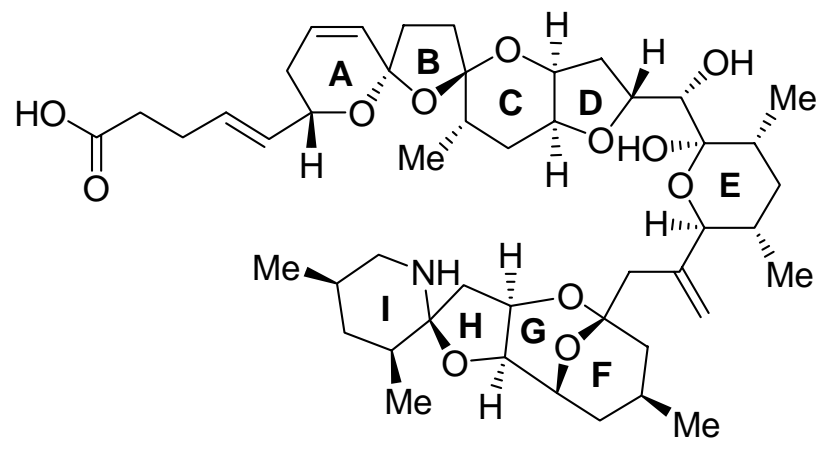

1b $0.045 \mathrm{mmol}, 1.0 \mathrm{M}$ in $\mathrm{H}_{2} \mathrm{O}, 10$ equiv). The reaction mixture was allowed to stir for $16 \mathrm{~h}$ at which time the starting material was consumed (TLC). The reaction mixture was then directly purified by preparative TLC (silica gel, $\mathrm{CH}_{3} \mathrm{Cl}: \mathrm{CH}_{3} \mathrm{OH}: \mathrm{H}_{2} \mathrm{O} 20: 3: 1$ ) to afford what is believed to be FGHI diastereomeric originally proposed azaspiracid-1 structure $\mathbf{1 b}$ as a colorless oil (1.4 mg, $0.0017 \mathrm{mmol}, 37 \%$ ) existing as an inseparable mixture of compounds. Further purification by HPLC using the conditions described in the isolation of azaspiracid- $1^{1}$ failed to yield a pure sample. 1b: $R_{\mathrm{f}}=0.48$ (silica gel, $\mathrm{CHCl}_{3}: \mathrm{MeOH}: \mathrm{H}_{2} \mathrm{O} 20: 3: 1$ ); HRMS (MALDI-FTMS) for $\mathrm{C}_{47} \mathrm{H}_{71} \mathrm{NO}_{12} \mathrm{H}^{+}\left[\mathrm{M}+\mathrm{H}^{+}\right]$ calcd 842.5049 , found 842.5058 . 
C-20-epi Alcohol 47. To a solution of triacetate 29 (12 mg, $0.017 \mathrm{mmol}, 1.0$ equiv) in $\mathrm{CH}_{2} \mathrm{Cl}_{2}(1 \mathrm{~mL})$ at $0{ }^{\circ} \mathrm{C}$ was added pyridine ( $0.3 \mathrm{~mL}, 0.34 \mathrm{mmol}, 20$ equiv), 4-DMAP ( $0.4 \mathrm{mg}, 0.003 \mathrm{mmol}, 0.2$ equiv), and

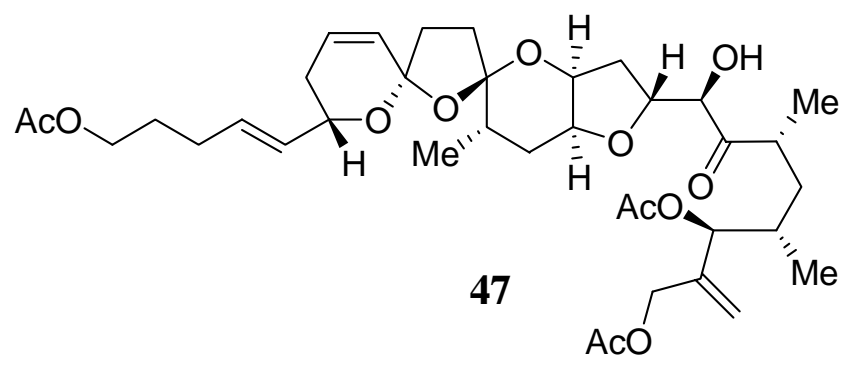
$\mathrm{Tf}_{2} \mathrm{O}(0.03 \mathrm{~mL}, 0.17 \mathrm{mmol}, 10$ equiv). The reaction mixture was allowed to stir at $0{ }^{\circ} \mathrm{C}$ for $1.5 \mathrm{~h}$. The reaction mixture was then quenched with saturated aqueous $\mathrm{NaHCO}_{3}(1 \mathrm{~mL})$ and extracted with $\mathrm{CH}_{2} \mathrm{Cl}_{2}$ $(3 \times 1 \mathrm{~mL})$, and the combined organic extracts were dried $\left(\mathrm{Na}_{2} \mathrm{SO}_{4}\right)$ and concentrated. The residue was then dissolved in DMF $(1 \mathrm{~mL})$ at $25{ }^{\circ} \mathrm{C}$. To the solution was added 18-crown-6 (4 mg, $0.085 \mathrm{mmol}, 5.0$ equiv) and $\mathrm{KNO}_{2}$ (33 mg, $0.085 \mathrm{mmol}, 5.0$ equiv), and the reaction mixture was allowed to react for $24 \mathrm{~h}$, at which time the reaction mixture was quenched with $\mathrm{H}_{2} \mathrm{O}(1 \mathrm{~mL})$. The resulting biphasic mixture was extracted with $\mathrm{E}_{2} \mathrm{O}(3 \times 2 \mathrm{~mL})$ and the combined organic layers were dried $\left(\mathrm{Na}_{2} \mathrm{SO}_{4}\right)$, concentrated, and then purified by flash column chromatography (silica gel, EtOAc:hexanes 1:2) to afford C-20-epi alcohol 47 (4 mg, 0.005 mmol, 31\% over two steps) as a colorless oil. 47: $R_{\mathrm{f}}=0.18$ (silica gel, EtOAc:hexanes 1:1); $[\alpha]_{\mathrm{D}}=-55.0(c=0.40$, $\mathrm{CHCl}_{3}$ ); IR (film) $v_{\max }=3448,2923,1738,1719,1701,1455,1373,1236 \mathrm{~cm}^{-1} ;{ }^{1} \mathrm{H} \mathrm{NMR}(600 \mathrm{MHz}$ $\left.\mathrm{CDCl}_{3}\right): \delta=6.00-5.97(\mathrm{~m}, 1 \mathrm{H}), 5.70-5.65(\mathrm{~m}, 2 \mathrm{H}), 5.53-5.50(\mathrm{~m}, 1 \mathrm{H}), 5.27(\mathrm{~s}, 1 \mathrm{H}), 5.17(\mathrm{~s}, 1 \mathrm{H})$, $5.02(\mathrm{~d}, J=7.0 \mathrm{~Hz}, 1 \mathrm{H}), 4.72-4.69(\mathrm{~m}, 1 \mathrm{H}), 4.53(\mathrm{~s}, 2 \mathrm{H}), 4.45(\mathrm{t}, J=4.4 \mathrm{~Hz}, 1 \mathrm{H}), 4.44-4.42(\mathrm{~m}, 1$ H), 4.16 (br s, $1 \mathrm{H}), 4.04$ (t, $J=6.6 \mathrm{~Hz}, 2 \mathrm{H}), 3.97$ (br s, $1 \mathrm{H}), 3.54$ (d, J=4.4 Hz, 1 H), 2.99-2.95 (m, 1 H), 2.22-2.18 (m, 1 H), 2.13-1.95 (m, $10 \mathrm{H}), 2.09$ (s, $3 \mathrm{H}), 2.08$ (s, $3 \mathrm{H}), 2.04$ (s, 3 H), 1.91-1.83 (m, 2 H), 1.72-1.64 (m, $3 \mathrm{H}), 1.48-1.44(\mathrm{~m}, 1 \mathrm{H}), 1.20-1.10(\mathrm{~m}, 1 \mathrm{H}), 1.07(\mathrm{~d}, J=6.6 \mathrm{~Hz}, 3 \mathrm{H}), 0.92(\mathrm{~d}, J=$ $6.6 \mathrm{~Hz}, 3 \mathrm{H}), 0.91(\mathrm{~d}, J=6.6 \mathrm{~Hz}, 3 \mathrm{H}) ;{ }^{13} \mathrm{C} \mathrm{NMR}\left(125 \mathrm{MHz}, \mathrm{CDCl}_{3}\right): \delta=214.5,171.1,170.5,170.2$, $140.7,131.1,130.8,129.0,128.6,116.5,111.4,104.2,79.7,78.6,78.2,76.6,76.1,68.8,63.9,63.8,39.7$ $36.9,35.7,34.2,33.7,33.2,30.9,30.0,29.7,28.7,28.0,23.3,21.0,20.9,17.5,16.5,15.6$; HRMS (MALDI-FTMS) for $\mathrm{C}_{37} \mathrm{H}_{54} \mathrm{O}_{12} \mathrm{Na}^{+}\left[\mathrm{M}+\mathrm{Na}^{+}\right]$calcd 713.3507, found 713.3511. 
C-20-epi Stille Product 48. C-20-epi triacetate 47 (50 mg, $0.072 \mathrm{mmol}, 1.0$ equiv) was azeotroped with benzene $(3 \times 5 \mathrm{~mL})$ and then dissolved in NMP $(2.5 \mathrm{~mL})$ at $25^{\circ} \mathrm{C} . \mathrm{LiCl}(19 \mathrm{mg}, 0.44 \mathrm{mmol}, 6.0$ equiv),

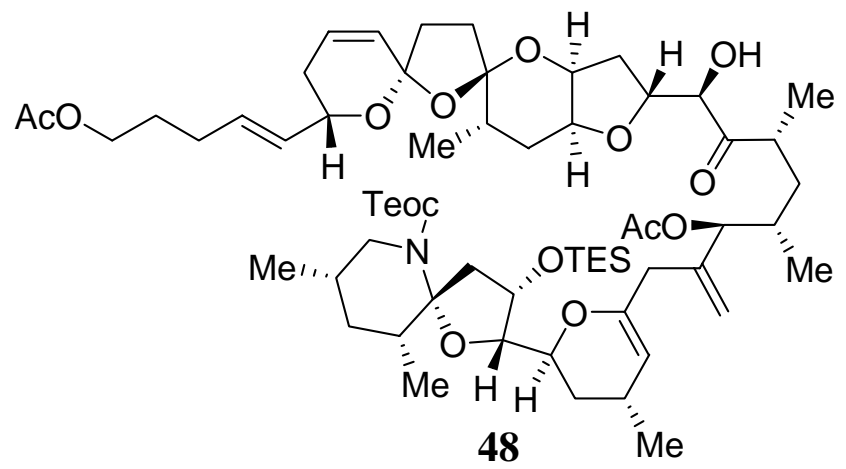
i-Pr $\operatorname{Pr}_{2} \mathrm{NEt}(0.075 \mathrm{~mL}, 0.44 \mathrm{mmol}, 6.0$ equiv) and $\mathrm{AsPh}_{3}(7 \mathrm{mg}, 0.022 \mathrm{mmol}, 0.3$ equiv) were added at $25{ }^{\circ} \mathrm{C}$ followed by $\mathrm{Pd}_{2} \mathrm{dba}_{3}(20 \mathrm{mg}, 0.022 \mathrm{mmol}, 0.3$ equiv). The reaction mixture was heated to $45^{\circ} \mathrm{C}$ and to this solution was added a solution of vinyl stannane 6 (153 mg, $0.22 \mathrm{mmol}, 3.0$ equiv) in THF (2.5 mL) over a period of $4 \mathrm{~h}$ via syringe pump. The reaction mixture was cooled to $25^{\circ} \mathrm{C}$ and quenched slowly with saturated aqueous $\mathrm{NaHCO}_{3}(2 \mathrm{~mL})$. The aqueous layer was extrated with EtOAc $(3 \times 10$ $\mathrm{mL}$ ) and the combined organic layers were washed with brine $(10 \mathrm{~mL})$, dried over $\mathrm{Na}_{2} \mathrm{SO}_{4}$, and concentrated. The crude product was purified by flash column chromatography (silica gel, hexanes:EtOAc 3:1) to afford C-20-epi Stille coupling product 48 (49 $\mathrm{mg}, 0.042 \mathrm{mmol}, 58 \%$ ) as a colorless oil. 48: $R_{\mathrm{f}}=0.56$ (silica gel, EtOAc:hexanes 1:1); $[\alpha]_{\mathrm{D}}=-26.7\left(c=2.1, \mathrm{CHCl}_{3}\right)$; IR $\left(\right.$ film) $v_{\max }$ $=2955,2875,1739,1701,1458,1395,1240 \mathrm{~cm}^{-1} ;{ }^{1} \mathrm{H}$ NMR $\left(600 \mathrm{MHz}, \mathrm{CDCl}_{3}\right): \delta=5.99-5.97(\mathrm{~m}, 1 \mathrm{H})$, 5.69-5.65 (m, $2 \mathrm{H}), 5.51(\mathrm{dd}, J=16.5,6.1 \mathrm{~Hz}, 1 \mathrm{H}), 5.29(\mathrm{~s}, 1 \mathrm{H}), 5.06(\mathrm{~s}, 1 \mathrm{H}), 5.02(\mathrm{~d}, J=6.1 \mathrm{~Hz}, 1$ H), 4.98 (s, 1 H), 4.72-4.69 (m, 1 H), 4.53-4.49 (m, 2 H), 4.46 (br s, 1 H), 4.42-4.40 (m, 1 H), 4.16 (br s, $1 \mathrm{H}), 4.14-4.07(\mathrm{~m}, 3 \mathrm{H}), 4.05(\mathrm{t}, J=6.6 \mathrm{~Hz}, 2 \mathrm{H}), 4.04-4.01(\mathrm{~m}, 1 \mathrm{H}), 3.96(\mathrm{br} \mathrm{s}, 1 \mathrm{H}), 3.68-3.65(\mathrm{~m}$, $1 \mathrm{H}) 3.56$ (br s, $1 \mathrm{H}), 3.07$ (t, $J=12.2 \mathrm{~Hz}, 1 \mathrm{H}), 2.99$ (s, $1 \mathrm{H}), 2.96-2.93(\mathrm{~m}, 1 \mathrm{H}), 2.90$ (dd, $J=14.5,6.6$ Hz, $1 \mathrm{H}), 2.71(\mathrm{~s}, 1 \mathrm{H}), 2.69$ (d, $J=16.0 \mathrm{~Hz}, 1 \mathrm{H}), 2.57$ (d, J=16.0 Hz, $1 \mathrm{H}), 2.47$ (dd, $J=14.5,6.6 \mathrm{~Hz}$ $1 \mathrm{H}), 2.28-2.16(\mathrm{~m}, 2 \mathrm{H}), 2.14-1.95(\mathrm{~m}, 4 \mathrm{H}), 2.06$ (s, $3 \mathrm{H}), 2.04(\mathrm{~s}, 3 \mathrm{H}), 1.90-1.75$ (m, $5 \mathrm{H}), 1.72-1.64$ (m, $3 \mathrm{H}), 1.58-1.50(\mathrm{~m}, 3 \mathrm{H}), 1.47-1.39(\mathrm{~m}, 2 \mathrm{H}), 1.32-1.22(\mathrm{~m}, 3 \mathrm{H}), 1.18-1.09(\mathrm{~m}, 2 \mathrm{H}), 1.05(\mathrm{~d}, J=$ $6.6 \mathrm{~Hz}, 3 \mathrm{H}), 0.97(\mathrm{~d}, J=6.6 \mathrm{~Hz}, 3 \mathrm{H}), 0.93(\mathrm{t}, J=7.6 \mathrm{~Hz}, 9 \mathrm{H}), 0.90(\mathrm{~d}, J=6.6 \mathrm{~Hz}, 3 \mathrm{H}), 0.88(\mathrm{~d}, J=$ $6.6 \mathrm{~Hz}, 3 \mathrm{H}), 0.79$ (d, $J=6.6 \mathrm{~Hz}, 6 \mathrm{H}), 0.57$ (q, $J=7.6 \mathrm{~Hz}, 6 \mathrm{H}), 0.02(\mathrm{~s}, 9 \mathrm{H}) ;{ }^{13} \mathrm{C} \mathrm{NMR}(125 \mathrm{MHz}$, $\left.\mathrm{CDCl}_{3}\right): \delta=214.6,171.1,170.0,156.1,150.5,142.7,131.1,130.8,129.0,128.6,114.2,104.2,111.4$ 104.1, 96.1, 83.1, 80.2, 79.6, 78.1, 76.6, 76.2, 72.0, 71.3, 68.8, 63.8, 62.8, 48.9, 39.8, 38.1, 37.1, 36.8, $35.7,34.1,33.7,32.6,30.9,30.6,30.0,29.7,28.6,28.2,28.0,24.6,23.3,22.8,21.1,21.0,18.6,17.6$, 
17.4, 16.7, 16.6, 15.5, 6.8, 4.6, -1.5; HRMS (MALDI-FTMS) for $\mathrm{C}_{63} \mathrm{H}_{103} \mathrm{NO}_{15} \mathrm{Si}_{2} \mathrm{Na}^{+}\left[\mathrm{M}+\mathrm{Na}^{+}\right]$calcd 1192.6758, found 1192.6797.

C-20-FGHI-epi Stille Product 49. C-20-epi triacetate 47 (50 mg, $0.072 \mathrm{mmol}, 1.0$ equiv) was azeotroped with benzene $(3 \times 5 \mathrm{~mL})$ and then dissolved in NMP $(2.5 \mathrm{~mL})$ at $25{ }^{\circ} \mathrm{C}$. $\mathrm{LiCl}(19 \mathrm{mg}, 0.44$

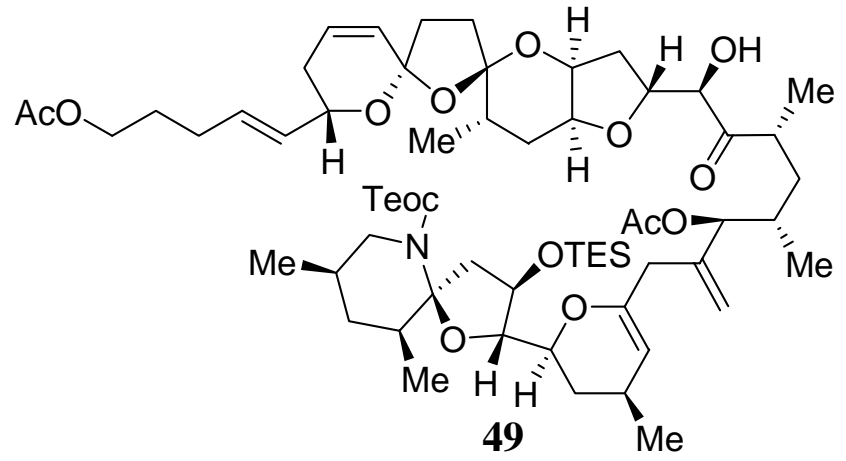

mmol, 6.0 equiv), $i-\operatorname{Pr}_{2} \mathrm{NEt}(0.075 \mathrm{~mL}, 0.44 \mathrm{mmol}$, 6.0 equiv) and $\mathrm{AsPh}_{3}$ (7 mg, $0.022 \mathrm{mmol}, 0.3$ equiv) were added at $25{ }^{\circ} \mathrm{C}$ followed by $\mathrm{Pd}_{2} \mathrm{dba}_{3}(20 \mathrm{mg}$, $0.022 \mathrm{mmol}, 0.3$ equiv). The reaction mixture was heated to $45{ }^{\circ} \mathrm{C}$ and to this solution was added dropwise a solution of vinyl stannane ent-6 (153 mg,

$0.22 \mathrm{mmol}, 3.0$ equiv) in THF (2.5 mL) over a period of $4 \mathrm{~h}$ via syringe pump. The reaction mixture was cooled to $25^{\circ} \mathrm{C}$ and quenched slowly with saturated aqueous $\mathrm{NaHCO}_{3}(2 \mathrm{~mL})$. The aqueous layer was extracted with EtOAc $(3 \times 10 \mathrm{~mL})$ and the combined organic layers were washed with brine $(10 \mathrm{~mL})$, dried over $\mathrm{Na}_{2} \mathrm{SO}_{4}$, and concentrated. The crude product was purified by flash column chromatography (silica gel, hexanes:EtOAc 3:1) to afford C-20-FGHI-epi Stille coupling product 49 (51 mg, 0.044 mmol, 61\%) as a colorless oil. 49: $R_{\mathrm{f}}=0.57$ (silica gel, EtOAc:hexanes 1:1); $[\alpha]_{\mathrm{D}}=-39.9(c=1.1$, $\mathrm{CHCl}_{3}$ ); IR (film) $v_{\max }=3448,2955,2913,1737,1697,1457,1390,1242,1172,1058 \mathrm{~cm}^{-1} ;{ }^{1} \mathrm{H}$ NMR $\left(500 \mathrm{MHz}, \mathrm{CDCl}_{3}\right): \delta=5.98-5.96(\mathrm{~m}, 1 \mathrm{H}), 5.69-5.65(\mathrm{~m}, 2 \mathrm{H}), 5.52(\mathrm{dd}, J=15.4,6.0 \mathrm{~Hz}, 1 \mathrm{H}), 5.06$ (s, $1 \mathrm{H}), 5.04(\mathrm{~d}, J=6.3 \mathrm{~Hz}, 1 \mathrm{H}), 4.99(\mathrm{~s}, 1 \mathrm{H}), 4.72-4.68(\mathrm{~m}, 1 \mathrm{H}), 4.52(\mathrm{~d}, J=3.7 \mathrm{~Hz}, 1 \mathrm{H}), 4.49-4.46$ (m, 2 H), 4.42-4.40 (m, $1 \mathrm{H}), 4.16-4.03$ (m, 8 H), 3.96 (br s, $1 \mathrm{H}), 3.68-3.65(\mathrm{~m}, 1 \mathrm{H}), 3.55$ (d, $J=4.5$ Hz, $1 \mathrm{H}), 3.07$ (t, $J=12.2 \mathrm{~Hz}, 1 \mathrm{H}), 2.99$ (s, $1 \mathrm{H}), 2.96-2.93(\mathrm{~m}, 1 \mathrm{H}), 2.90$ (dd, J = 14.5, 6.6 Hz, $1 \mathrm{H})$, $2.71(\mathrm{~s}, 1 \mathrm{H}), 2.69(\mathrm{~d}, J=16.0 \mathrm{~Hz}, 1 \mathrm{H}), 2.57(\mathrm{~d}, J=16.0 \mathrm{~Hz}, 1 \mathrm{H}), 2.47(\mathrm{dd}, J=14.5,6.6 \mathrm{~Hz}, 1 \mathrm{H})$, 2.28-2.16 (m, 2 H), 2.14-1.95 (m, 4 H), 2.06 (s, 3 H), 2.04 (s, 3 H), 1.90-1.75 (m, 5 H), 1.72-1.64 (m, 3 H), $1.58-1.50(\mathrm{~m}, 3 \mathrm{H}), 1.47-1.39(\mathrm{~m}, 2 \mathrm{H}), 1.32-1.22(\mathrm{~m}, 3 \mathrm{H}), 1.18-1.09(\mathrm{~m}, 2 \mathrm{H}), 1.05(\mathrm{~d}, J=6.6 \mathrm{~Hz}$ $3 \mathrm{H}), 0.97(\mathrm{~d}, J=6.6 \mathrm{~Hz}, 3 \mathrm{H}), 0.93(\mathrm{t}, J=7.6 \mathrm{~Hz}, 9 \mathrm{H}), 0.90(\mathrm{~d}, J=6.6 \mathrm{~Hz}, 3 \mathrm{H}), 0.88(\mathrm{~d}, J=6.6 \mathrm{~Hz}, 3$ $\mathrm{H}), 0.79(\mathrm{~d}, J=6.6 \mathrm{~Hz}, 6 \mathrm{H}), 0.57(\mathrm{q}, J=7.6 \mathrm{~Hz}, 6 \mathrm{H}), 0.02(\mathrm{~s}, 9 \mathrm{H}) ;{ }^{13} \mathrm{C} \mathrm{NMR}\left(125 \mathrm{MHz}, \mathrm{CDCl}_{3}\right): \delta=$ 
215.0, 171.5, 170.4, 156.5, 150.5, 142.9, 131.4, 131.2, 129.3, 129.0, 114.7, 111.8, 104.6, 104.5, 96.5, $83.3,80.4,80.0,78.5,76.9,76.5,72.3,71.3,69.2,64.2,63.2,49.8,40.1,38.6,38.0,37.7,37.1,36.0$, $34.5,34.0,33.0,31.5,31.2,31.0,30.4,30.0,29.9,29.0,28.3,24.8,23.7,23.1,21.4,21.3,19.0,18.0$, 17.1, 17.0, 15.9, 7.2, 5.0, -1.1; HRMS (MALDI-FTMS) for $\mathrm{C}_{63} \mathrm{H}_{103} \mathrm{NO}_{15} \mathrm{Si}_{2} \mathrm{Na}^{+}\left[\mathrm{M}+\mathrm{Na}^{+}\right]$calcd 1192.6758, found 1192.6794 .

C-20-epi Des-iodo product 54. C-20-epi Stille coupling product 48 (60 mg, $0.051 \mathrm{mmol}, 1.0$ equiv) was dissolved in a mixture of pyridine:THF $(1: 1,3 \mathrm{~mL})$ and cooled to $0{ }^{\circ} \mathrm{C}$. HF $\bullet$ py $(0.15 \mathrm{~mL})$ was added

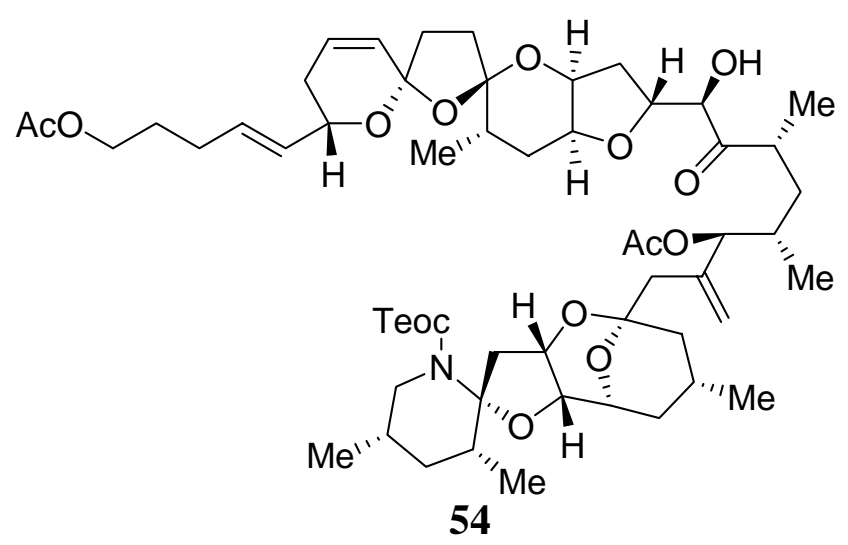
dropwise and the reaction mixture was stirred at 25 ${ }^{\circ} \mathrm{C}$ for $2.5 \mathrm{~h}$ and then quenched slowly with saturated aqueous $\mathrm{NaHCO}_{3}(5 \mathrm{~mL})$ at $0{ }^{\circ} \mathrm{C}$. The aqueous layer was extracted with EtOAc $(3 \times 10 \mathrm{~mL})$ and the combined organic layers were dried over $\mathrm{Na}_{2} \mathrm{SO}_{4}$, filtered and concentrated. The crude product was dissolved in THF $(6 \mathrm{~mL})$ and cooled to $0{ }^{\circ} \mathrm{C}$.

$\mathrm{NaHCO}_{3}$ (130 mg, $1.5 \mathrm{mmol}, 30$ equiv) was added followed by NIS (115 mg, $0.51 \mathrm{mmol}, 10$ equiv). The reaction mixture was stirred at $0{ }^{\circ} \mathrm{C}$ overnight and then quenched slowly with saturated aqueous $\mathrm{Na}_{2} \mathrm{~S}_{2} \mathrm{O}_{3}(5 \mathrm{~mL})$ at $0{ }^{\circ} \mathrm{C}$. The biphasic mixture was extracted with EtOAc $(3 \times 10 \mathrm{~mL})$ and the combined organic layers were dried over $\mathrm{Na}_{2} \mathrm{SO}_{4}$, concentrated and carried on to the next step without further purification. C-20-epi octacycle 52 (21 $\mathrm{mg}, 0.018 \mathrm{mmol}, 1.0$ equiv) was dissolved in a mixture of $n$ $\mathrm{Bu}_{3} \mathrm{SnH}$ :toluene $(1: 2,1.5 \mathrm{~mL})$ and cooled to $0{ }^{\circ} \mathrm{C}$. $\mathrm{Et}_{3} \mathrm{~B}(1.0 \mathrm{M}$ in hexanes, 1 drop) was added and the reaction mixture was stirred for $5 \mathrm{~min}$. The reaction mixture was quenched with $\mathrm{H}_{2} \mathrm{O}(2 \mathrm{~mL})$. The aqueous layer was extracted with EtOAc $(3 \times 5 \mathrm{~mL})$ and the combined organic layers were washed with saturated aqueous $\mathrm{NaCl}(10 \mathrm{~mL})$, dried over $\mathrm{Na}_{2} \mathrm{SO}_{4}$, and concentrated. The crude product was purified by flash column chromatography (silica gel, hexanes:EtOAc 4:1) to afford C-20-epi des-iodo product 54 (30 mg, $0.028 \mathrm{mmol}, 55 \%$ over three steps) as a colorless oil. 54: $R_{\mathrm{f}}=0.24$ (silica gel, $\mathrm{CH}_{2} \mathrm{Cl}_{2}$ :acetone 
19:1); $[\alpha]_{\mathrm{D}}=-29.8\left(c=0.6, \mathrm{CHCl}_{3}\right) ; \mathrm{IR}($ film $) v_{\max }=3396,2955,2921,2853,1739,1696,1454,1243$, $1057 \mathrm{~cm}^{-1} ;{ }^{1} \mathrm{H}$ NMR (500 MHz, $\left.\mathrm{CDCl}_{3}\right): \delta=6.00-5.96(\mathrm{~m}, 1 \mathrm{H}), 5.70-5.65(\mathrm{~m}, 2 \mathrm{H}), 5.51(\mathrm{dd}, J=$ 16.0, $6.3 \mathrm{~Hz}, 1 \mathrm{H}), 5.17(\mathrm{~s}, 1 \mathrm{H}), 5.13$ (d, $J=5.5 \mathrm{~Hz}, 1 \mathrm{H}), 5.03$ (s, $1 \mathrm{H}), 4.72-4.68$ (m, $2 \mathrm{H}), 4.49$ (t, $J=$ $4.0 \mathrm{~Hz}, 1 \mathrm{H}), 4.44-4.40(\mathrm{~m}, 1 \mathrm{H}), 4.26(\mathrm{~d}, J=4.4 \mathrm{~Hz}, 1 \mathrm{H}), 4.20-4.14(\mathrm{~m}, 2 \mathrm{H}), 4.05$ (t, J=6.5 Hz, $2 \mathrm{H})$, 4.04-4.00 (m, 1 H), 3.97-3.96 (m, 1 H), 3.86 (d, J = 4.4 Hz, 1 H), 3.76-3.73 (m, 2 H), 3.65-3.62 (m, 1 H), 3.20 (dd, $J=13.2,12.0 \mathrm{~Hz}, 1 \mathrm{H}), 2.94-2.90$ (m, $1 \mathrm{H}), 2.35$ (s, $1 \mathrm{H}), 2.27-1.95$ (m, $11 \mathrm{H}), 2.08$ (s, 3 H), $2.04(\mathrm{~s}, 3 \mathrm{H}), 1.87-1.81(\mathrm{~m}, 3 \mathrm{H}), 1.73-1.67(\mathrm{~m}, 3 \mathrm{H}), 1.66-1.40(\mathrm{~m}, 10 \mathrm{H}), 1.03(\mathrm{~d}, J=6.6 \mathrm{~Hz}, 3$ H), 1.01-0.94 (m, $4 \mathrm{H}), 0.91(\mathrm{~d}, J=6.5 \mathrm{~Hz}, 3 \mathrm{H}), 0.89(\mathrm{~d}, J=6.5 \mathrm{~Hz}, 3 \mathrm{H}), 0.85(\mathrm{~d}, J=6.5 \mathrm{~Hz}, 3 \mathrm{H})$, $0.81(\mathrm{~d}, J=6.5 \mathrm{~Hz}, 3 \mathrm{H}), 0.79(\mathrm{~m}, 6.5 \mathrm{~Hz}, 3 \mathrm{H}), 0.03(\mathrm{~s}, 9 \mathrm{H}) ;{ }^{13} \mathrm{C} \mathrm{NMR}\left(125 \mathrm{MHz}, \mathrm{CDCl}_{3}\right): \delta=214.2$, 171.1, 170.2, 156.1, 140.4, 131.1, 130.9, 129.1, 128.5, 117.1, 111.4, 104.2, 97.0, 96.3, 80.8, 79.5, 78.1, $78.0,76.5,76.3,73.4,71.7,68.8,63.8,62.8,48.8,44.6,41.2,39.9,39.8,38.9,36.6,36.3,35.7,35.2$, $34.0,33.7,31.9,31.4,30.9,30.0,29.7,28.7,28.0,24.3,23.3,23.0,21.2,21.0,18.7,17.8,17.3,16.5$, 15.6, -1.5; HRMS (MALDI-FTMS) for $\mathrm{C}_{57} \mathrm{H}_{89} \mathrm{NO}_{15} \mathrm{SiNa}^{+}\left[\mathrm{M}+\mathrm{Na}^{+}\right]$calcd 1078.5893, found 1078.5902 .

C-20-FGHI-epi Des-iodo Product 55. C-20-FGHI-epi Stille coupling product 49 (60 mg, $0.051 \mathrm{mmol}$, 1.0 equiv) was dissolved in a mixture of pyridine:THF $(1: 1,3 \mathrm{~mL})$ and cooled to $0{ }^{\circ} \mathrm{C}$. HF $\bullet$ py $(0.15 \mathrm{~mL})$

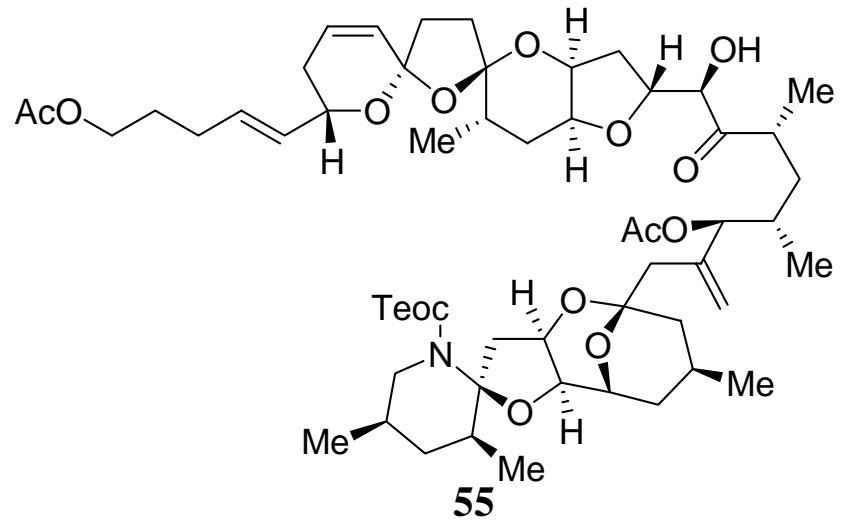
was added dropwise and the reaction mixture was stirred at $25{ }^{\circ} \mathrm{C}$ for $2.5 \mathrm{~h}$ and then quenched slowly with saturated aqueous $\mathrm{NaHCO}_{3}(5 \mathrm{~mL})$ at $0{ }^{\circ} \mathrm{C}$. The aqueous layer was extracted with EtOAc $(3 \times 10 \mathrm{~mL})$ and the combined organic layers were dried over $\mathrm{Na}_{2} \mathrm{SO}_{4}$, filtered and concentrated. The crude product was dissolved in THF $(6 \mathrm{~mL})$ and cooled to $0{ }^{\circ} \mathrm{C}$. $\mathrm{NaHCO}_{3}$ (130 mg, $1.5 \mathrm{mmol}, 30$ equiv) was added followed by NIS (115 mg, $0.51 \mathrm{mmol}, 10$ equiv). The reaction mixture was stirred at $0{ }^{\circ} \mathrm{C}$ overnight and then quenched slowly with saturated aqueous $\mathrm{Na}_{2} \mathrm{~S}_{2} \mathrm{O}_{3}(5 \mathrm{~mL})$ at $0{ }^{\circ} \mathrm{C}$. The aqueous layer was extracted with EtOAc $(3 \times 10 \mathrm{~mL})$ and the combined 
organic layers were dried over $\mathrm{Na}_{2} \mathrm{SO}_{4}$, filtered, concentrated, and used in the next step without further purification. C-20-FGHI-epi diastereomeric octacycle 53 (21 mg, $0.018 \mathrm{mmol}, 1.0$ equiv) was dissolved in a mixture of $n-\mathrm{Bu}_{3} \mathrm{SnH}$ :toluene $(1: 2,1.5 \mathrm{~mL})$ and cooled to $0{ }^{\circ} \mathrm{C}$. $\mathrm{Et}_{3} \mathrm{~B}(1.0 \mathrm{M}$ in hexanes, 1 drop) was added and the reaction mixture was stirred for 5 min. The reaction mixture was quenched with $\mathrm{H}_{2} \mathrm{O}(2$ $\mathrm{mL})$. The aqueous layer was extracted with EtOAc $(3 \times 5 \mathrm{~mL})$ and the combined organic layers were washed with saturated aqueous $\mathrm{NaCl}(10 \mathrm{~mL})$, dried over $\mathrm{Na}_{2} \mathrm{SO}_{4}$, and concentrated. The crude product was purified by flash column chromatography (silica gel, hexanes:EtOAc 4:1) to afford C-20-FGHI-epi des-iodo product 55 (27 mg, $0.026 \mathrm{mmol}, 51 \%$ over three steps) as a colorless oil. 55: $R_{\mathrm{f}}=0.39$ (silica gel, EtOAc 3:4); $[\alpha]_{\mathrm{D}}=-8.0\left(c=0.45, \mathrm{CHCl}_{3}\right)$; IR (film) $v_{\max }=3441,2955,2930,1739,1690,1455$, 1392, 1362, 1243, 1167, $1056 \mathrm{~cm}^{-1} ;{ }^{1} \mathrm{H}$ NMR (500 MHz, $\left.\mathrm{CDCl}_{3}\right): \delta=6.02-5.95(\mathrm{~m}, 1 \mathrm{H}), 5.74-5.64(\mathrm{~m}$, $2 \mathrm{H}), 5.55-5.49$ (m, $1 \mathrm{H}), 5.29$ (br s, $1 \mathrm{H}), 5.52(\mathrm{dd}, J=12.8,2.0 \mathrm{~Hz}, 1 \mathrm{H}), 5.29(\mathrm{~s}, 1 \mathrm{H}), 5.02(\mathrm{~s}, 1 \mathrm{H})$, 4.96 (s, $1 \mathrm{H}), 4.77-4.69$ (m, 2 H), 4.52 (br s, $1 \mathrm{H}), 4.45-4.39$ (m, $1 \mathrm{H}), 4.31-4.26$ (m, $1 \mathrm{H}), 4.20-4.06$ $(\mathrm{m}, 3 \mathrm{H}), 4.05(\mathrm{t}, J=8.0 \mathrm{~Hz}, 2 \mathrm{H}), 3.98(\mathrm{~s}, 1 \mathrm{H}), 3.83-3.72(\mathrm{~m}, 3 \mathrm{H}), 3.40-3.30(\mathrm{~m}, 1 \mathrm{H}), 3.18(\mathrm{t}, J=$ $12.0 \mathrm{~Hz}, 1 \mathrm{H}), 2.94-2.90$ (m, $1 \mathrm{H}), 2.35$ (s, $1 \mathrm{H}), 2.27-1.95$ (m, $11 \mathrm{H}), 2.08$ (s, $3 \mathrm{H}), 2.04$ (s, $3 \mathrm{H}), 1.87-$ $1.81(\mathrm{~m}, 3 \mathrm{H}), 1.73-1.67(\mathrm{~m}, 3 \mathrm{H}), 1.66-1.40(\mathrm{~m}, 10 \mathrm{H}), 1.03$ (d, J=6.6 Hz, $3 \mathrm{H}), 1.01-0.94(\mathrm{~m}, 4 \mathrm{H})$, $0.91(\mathrm{~d}, J=6.5 \mathrm{~Hz}, 3 \mathrm{H}), 0.89(\mathrm{~d}, J=6.5 \mathrm{~Hz}, 3 \mathrm{H}), 0.85(\mathrm{~d}, J=6.5 \mathrm{~Hz}, 3 \mathrm{H}), 0.81(\mathrm{~d}, J=6.5 \mathrm{~Hz}, 3 \mathrm{H})$, $0.79(\mathrm{~m}, J=6.5 \mathrm{~Hz}, 3 \mathrm{H}), 0.03(\mathrm{~s}, 9 \mathrm{H}) ;{ }^{13} \mathrm{C} \mathrm{NMR}\left(125 \mathrm{MHz}, \mathrm{CDCl}_{3}\right): \delta=214.7,171.5,170.4,156.2$, $142.4,131.5,131.2,129.4,128.9,115.7,111.8,104.5,97.4,96.5,80.1,80.0,78.4,76.9,76.6,74.0,71.5$ $69.2,64.2,63.2,48.7,46.3,46.2,40.8,39.9,39.7,39.5,36.9,36.1,35.8,34.9,34.4,34.0,31.9,31.8$, $31.2,30.4,30.0,29.0,28.4,25.0,23.7,23.3,21.5,21.3,19.0,18.3,17.7,16.8,15.9,-1.1$; HRMS (MALDI-FTMS) for $\mathrm{C}_{57} \mathrm{H}_{89} \mathrm{NO}_{15} \mathrm{SiNa}^{+}\left[\mathrm{M}+\mathrm{Na}^{+}\right]$calcd 1078.5893, found 1078.5879.

C-20-epi Primary Alcohol 58. C-20-epi Des-iodo Product 54 (16 mg, 0.014 mmol, 1.0 equiv) was dissolved in $\mathrm{CH}_{2} \mathrm{Cl}_{2}(3 \mathrm{~mL})$ and cooled to $-78{ }^{\circ} \mathrm{C}$. 2,6-Lutidine (0.050 mL, $0.28 \mathrm{mmol}, 20$ equiv) was added, followed by TESOTf $\left(0.050 \mathrm{~mL}, 0.14 \mathrm{mmol}, 10\right.$ equiv). After being stirred for $10 \mathrm{~min}$ at $0{ }^{\circ} \mathrm{C}$ the reaction mixture was quenched by the addition of saturated aqueous $\mathrm{NH}_{4} \mathrm{Cl}(5 \mathrm{~mL})$ and the two layers were separated. The aqueous layer was extracted with $\mathrm{CH}_{2} \mathrm{Cl}_{2}(3 \times 10 \mathrm{~mL})$ and the combined organic 
layers were washed with $\mathrm{H}_{2} \mathrm{O}(10 \mathrm{~mL})$ and saturated aqueous $\mathrm{NaCl}(10 \mathrm{~mL})$, dried over $\mathrm{Na}_{2} \mathrm{SO}_{4}$, filtered, and concentrated. The crude material was dissolved in $\mathrm{MeOH}(2.5 \mathrm{~mL})$ at $25{ }^{\circ} \mathrm{C} . \mathrm{K}_{2} \mathrm{CO}_{3}(13$

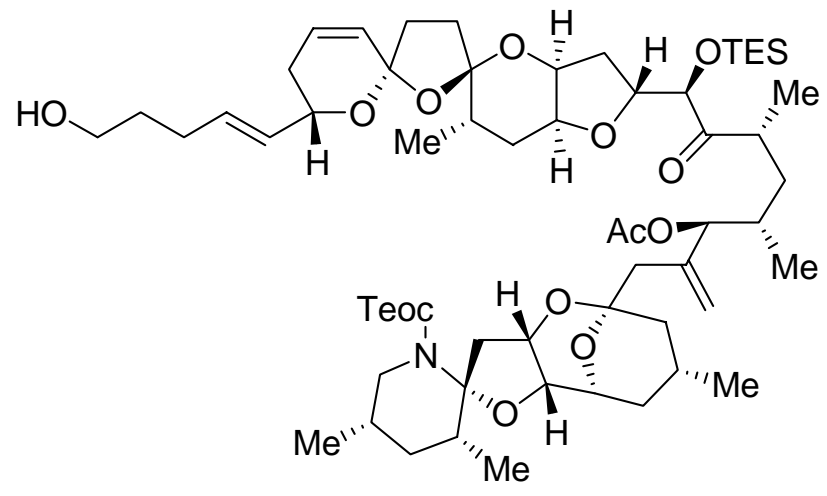

58 mg, 0.009 mmol, 1.0 equiv) was added and the reaction mixture was stirred at $25{ }^{\circ} \mathrm{C}$ for $2 \mathrm{~h}$. The reaction was diluted with $\mathrm{H}_{2} \mathrm{O}(5 \mathrm{~mL})$ and extracted with EtOAc $(3 \times 5 \mathrm{~mL})$. The combined organic layers were washed with brine $(10 \mathrm{~mL})$, dried over $\mathrm{Na}_{2} \mathrm{SO}_{4}$, and concentrated. The crude product was purified by flash column chromatography (silica gel, hexanes:EtOAc 2:1) to afford C-20-epi primary alcohol 58 (12 mg, $0.011 \mathrm{mmol}, 76 \%$ over two steps) as a colorless oil. 58: $R_{\mathrm{f}}=0.26$ (silica gel, EtOAc:hexanes 4:6); $[\alpha]_{\mathrm{D}}=-8.7\left(c=0.3, \mathrm{CHCl}_{3}\right) ; \mathrm{IR}($ film) $v_{\max }=3414,2955,2920,2849,1738,1729,1694,1451,1382,1244 \mathrm{~cm}^{-1} ;{ }^{1} \mathrm{H}$ NMR $\left(500 \mathrm{MHz}, \mathrm{CDCl}_{3}\right)$ : $\delta=6.00-5.96(\mathrm{~m}, 1 \mathrm{H}), 5.74-5.68(\mathrm{~m}, 2 \mathrm{H}), 5.53(\mathrm{dd}, J=15.5,6.3 \mathrm{~Hz}, 1 \mathrm{H}), 5.27(\mathrm{~s}, 1 \mathrm{H}), 5.10(\mathrm{~s}, 1 \mathrm{H})$, $5.03(\mathrm{~d}, J=7.0 \mathrm{~Hz}, 1 \mathrm{H}), 4.73-4.66(\mathrm{~m}, 2 \mathrm{H}), 4.47$ (d, $J=2.6 \mathrm{~Hz}, 1 \mathrm{H}), 4.44-4.40(\mathrm{~m}, 1 \mathrm{H}), 4.29(\mathrm{~d}, J=$ $4.8 \mathrm{~Hz}, 1 \mathrm{H}), 4.18-4.13(\mathrm{~m}, 2 \mathrm{H}), 4.01-3.96(\mathrm{~m}, 1 \mathrm{H}), 3.82-3.72(\mathrm{~m}, 3 \mathrm{H}), 3.64$ (t, J = 6.2 Hz, $2 \mathrm{H}), 3.22$ (t, $J=12.1 \mathrm{~Hz}, 1 \mathrm{H}), 2.92$ (q, $J=7.0 \mathrm{~Hz}, 1 \mathrm{H}), 2.25$ (d, $J=14.2 \mathrm{~Hz}, 1 \mathrm{H}), 2.19-2.11(\mathrm{~m}, 6 \mathrm{H}), 2.06-1.91$ $(\mathrm{m}, 11 \mathrm{H}), 1.87-1.78(\mathrm{~m}, 3 \mathrm{H}), 1.68-1.64(\mathrm{~m}, 6 \mathrm{H}), 1.50-1.42(\mathrm{~m}, 3 \mathrm{H}), 1.34-1.15(\mathrm{~m}, 8 \mathrm{H}), 0.99(\mathrm{~d}, J=$ $6.5 \mathrm{~Hz}, 3 \mathrm{H}), 0.97(\mathrm{~d}, J=6.5 \mathrm{~Hz}, 3 \mathrm{H}), 0.96(\mathrm{t}, J=8.0 \mathrm{~Hz}, 9 \mathrm{H}), 0.90(\mathrm{~d}, J=6.5 \mathrm{~Hz}, 3 \mathrm{H}), 0.88(\mathrm{~d}, J=$ $6.5 \mathrm{~Hz}, 3 \mathrm{H}), 0.85(\mathrm{~d}, J=6.5 \mathrm{~Hz}, 3 \mathrm{H}), 0.83(\mathrm{~d}, J=6.5 \mathrm{~Hz}, 3 \mathrm{H}), 0.61(\mathrm{q}, J=8.0 \mathrm{~Hz}, 6 \mathrm{H}), 0.02(\mathrm{~s}, 9 \mathrm{H})$; ${ }^{13} \mathrm{C}$ NMR $\left(125 \mathrm{MHz}, \mathrm{CDCl}_{3}\right): \delta=213.5,170.2,156.0,140.2,131.9,130.6,129.1,128.5,118.2,111.4$, 104.1, 97.0, 96.0, 81.8, 80.4, 79.0, 78.1, 76.5, 76.3, 73.5, 71.0, 68.8, 62.7, 62.4, 48.6, 43.7, 41.2, 39.5, $39.1,36.5,36.4,35.7,35.0,33.9,33.8,32.6,32.0,31.4,30.9,30.0,29.7,29.4,28.7,24.7,23.3,21.3$, 18.7, 17.81, 17.75, 17.5, 16.5, 15.6, 6.8, 4.9, -1.5; HRMS (MALDI-FTMS) for $\mathrm{C}_{61} \mathrm{H}_{101} \mathrm{NO}_{14} \mathrm{Si}_{2} \mathrm{Na}^{+}[\mathrm{M}$ $+\mathrm{Na}^{+}$] calcd 1150.6652, found 1150.6629.

C-20-FGHI-epi Primary Alcohol 59. C-20-FGHI-epi des-iodo product 55 (16 mg, 0.014 mmol, 1.0 equiv) was dissolved in $\mathrm{CH}_{2} \mathrm{Cl}_{2}(3 \mathrm{~mL})$ and cooled to $-78^{\circ} \mathrm{C} .2,6$-Lutidine $(0.050 \mathrm{~mL}, 0.28 \mathrm{mmol}, 20$ 
equiv) was added, followed by TESOTf ( $0.050 \mathrm{~mL}, 0.14 \mathrm{mmol}, 10$ equiv). After being stirred for $10 \mathrm{~min}$ at $0{ }^{\circ} \mathrm{C}$ the reaction mixture was quenched by the addition of saturated aqueous $\mathrm{NH}_{4} \mathrm{Cl}(5 \mathrm{~mL})$ and the

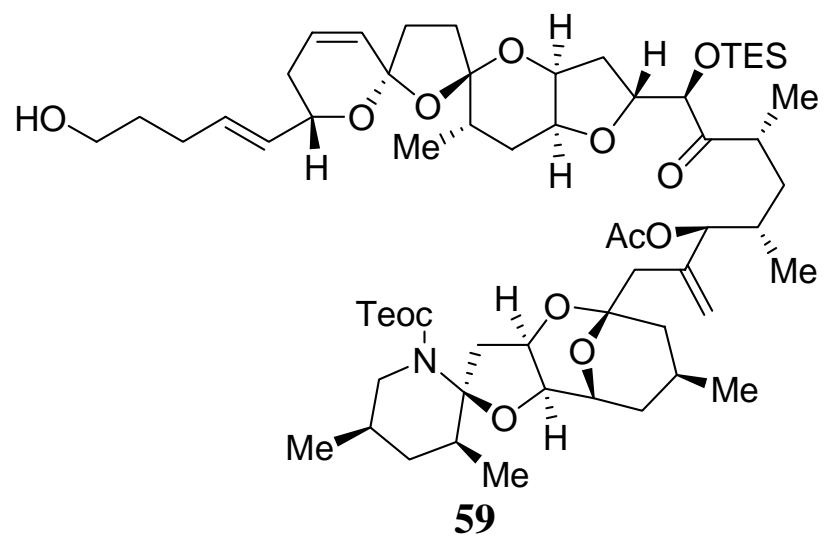
two layers were separated. The aqueous layer was extracted with $\mathrm{CH}_{2} \mathrm{Cl}_{2}(3 \times 10 \mathrm{~mL})$ and the combined organic layers were washed with $\mathrm{H}_{2} \mathrm{O}(10 \mathrm{~mL})$ and saturated aqueous $\mathrm{NaCl}(10 \mathrm{~mL})$. The combined organic layers were dried over $\mathrm{Na}_{2} \mathrm{SO}_{4}$, filtered, and concentrated. The crude material was dissolved in $\mathrm{MeOH}(2.5 \mathrm{~mL})$ at $25{ }^{\circ} \mathrm{C} . \mathrm{K}_{2} \mathrm{CO}_{3}(13 \mathrm{mg}, 0.009$ mmol, 1.0 equiv) was added and the reaction mixture was stirred at $25^{\circ} \mathrm{C}$ for $2 \mathrm{~h}$. The reaction mixture was diluted with $\mathrm{H}_{2} \mathrm{O}(5 \mathrm{~mL})$ and extracted with EtOAc $(3 \times 5 \mathrm{~mL})$. The combined organic layers were washed with brine $(10 \mathrm{~mL})$, dried over $\mathrm{Na}_{2} \mathrm{SO}_{4}$, and concentrated. The crude product was purified by flash column chromatography (silica gel, hexanes:EtOAc 2:1) to afford C-20-FGHI-epi free alcohol 59 (13 mg, $0.011 \mathrm{mmol}, 76 \%$ over two steps) as a colorless oil. 59: $R_{\mathrm{f}}=0.31$ (silica gel, EtOAc:hexanes 2:3); $[\alpha]_{\mathrm{D}}=-20.3\left(c=0.3, \mathrm{CHCl}_{3}\right) ; \mathrm{IR}($ film $) v_{\max }=\mathrm{cm}^{-1} ;{ }^{1} \mathrm{H} \mathrm{NMR}\left(600 \mathrm{MHz}, \mathrm{CDCl}_{3}\right): \delta=5.98-5.96$ (m, $1 \mathrm{H}), 5.73-5.68(\mathrm{~m}, 2 \mathrm{H}), 5.52(\mathrm{dd}, J=13.2,5.0 \mathrm{~Hz}, 1 \mathrm{H}), 5.21(\mathrm{~d}, J=4.0 \mathrm{~Hz}, 1 \mathrm{H}), 5.14(\mathrm{~s}, 1 \mathrm{H})$, $5.05(\mathrm{~s}, 1 \mathrm{H}), 4.76-4.72(\mathrm{~m}, 1 \mathrm{H}), 4.70-4.66(\mathrm{~m}, 1 \mathrm{H}), 4.49$ (d, J = 2.2 Hz, $1 \mathrm{H}), 4.44-4.40(\mathrm{~m}, 1 \mathrm{H})$, 4.30 (d, $J=3.6 \mathrm{~Hz}, 1 \mathrm{H}), 4.19-4.11(\mathrm{~m}, 2 \mathrm{H}), 4.06-4.01(\mathrm{~m}, 1 \mathrm{H}), 3.82-3.73$ (m, $2 \mathrm{H}), 3.64$ (t, $J=7.0$ Hz, 2 H), 3.39-3.27 (m, 1 H), 3.21 (t, $J=11.8 \mathrm{~Hz}, 1 \mathrm{H}), 2.94$ (q, $J=6.6 \mathrm{~Hz}, 1 \mathrm{H}), 2.25$ (d, $J=14.2 \mathrm{~Hz}$, $1 \mathrm{H}), 2.19-2.11$ (m, $6 \mathrm{H}), 2.06-1.91(\mathrm{~m}, 11 \mathrm{H}), 1.87-1.78$ (m, $3 \mathrm{H}), 1.68-1.64$ (m, $6 \mathrm{H}), 1.50-1.42$ (m, 3 H), 1.34-1.15 (m, 8 H), 0.99 (d, $J=6.5 \mathrm{~Hz}, 3 \mathrm{H}), 0.97$ (d, $J=6.5 \mathrm{~Hz}, 3 \mathrm{H}), 0.96$ (t, $J=8.0 \mathrm{~Hz}, 9 \mathrm{H})$, $0.90(\mathrm{~d}, J=6.5 \mathrm{~Hz}, 3 \mathrm{H}), 0.88$ (d, $J=6.5 \mathrm{~Hz}, 3 \mathrm{H}), 0.85$ (d, $J=6.5 \mathrm{~Hz}, 3 \mathrm{H}), 0.83$ (d, $J=6.5 \mathrm{~Hz}, 3 \mathrm{H})$, $0.61(\mathrm{q}, J=8.0 \mathrm{~Hz}, 6 \mathrm{H}), 0.02(\mathrm{~s}, 9 \mathrm{H}) ;{ }^{13} \mathrm{C}$ NMR $\left(125 \mathrm{MHz}, \mathrm{CDCl}_{3}\right): \delta=213.8,170.4,156.3,142.3$, 132.3, 130.9, 129.5, 128.8, 116.5, 111.8, 104.5, 97.4, 96.6, 80.7, 80.6, 79.3, 78.4, 76.8, 76.7, 74.0, 71.5, 69.2, 63.0, 62.7, 48.9, 45.5, 41.1, 39.7, 39.4, 36.9, 36.4, 36.1, 35.0, 34.3, 34.2, 33.4, 32.4, 31.8, 31.3, $30.4,30.1,29.1,24.9,23.7,23.4,21.5,19.1,18.3,18.2,17.7,16.9,15.9,7.2,5.3,-1.1$; HRMS (MALDI-FTMS) for $\mathrm{C}_{61} \mathrm{H}_{101} \mathrm{NO}_{14} \mathrm{Si}_{2} \mathrm{Na}^{+}\left[\mathrm{M}+\mathrm{Na}^{+}\right]$calcd 1150.6652, found 1150.6675 . 
C-20-epi Free Amine 64. To a solution of oxalyl chloride $\left(2 \mu \mathrm{L}, 0.12 \mathrm{mmol}, 10.0\right.$ equiv) in $\mathrm{CH}_{2} \mathrm{Cl}_{2}(1$ $\mathrm{mL})$ at $-78{ }^{\circ} \mathrm{C}$ was added DMSO $(15 \mu \mathrm{L}, 0.24 \mathrm{mmol}, 20.0$ equiv). The solution was allowed to stir for

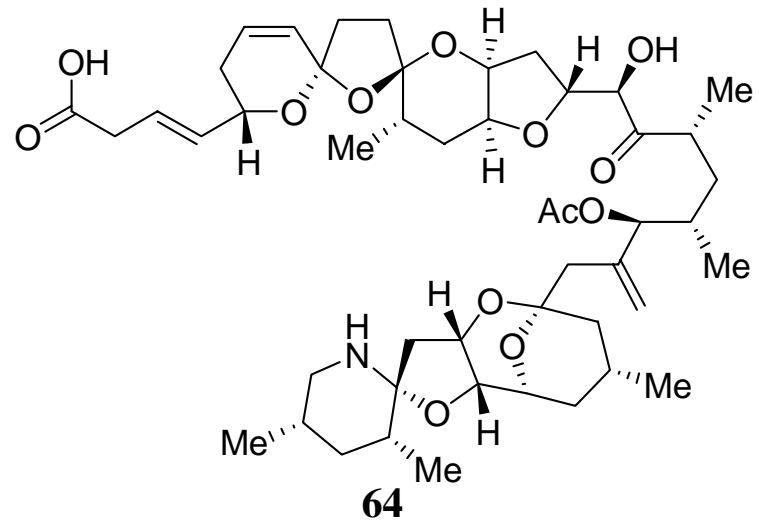
$20 \mathrm{~min}$ at $-78^{\circ} \mathrm{C}$ and then a solution of C-20-epi primary alcohol 58 (13.5 mg, $0.012 \mathrm{mmol}, 1.0$ equiv) in $\mathrm{CH}_{2} \mathrm{Cl}_{2}$ $(0.5 \mathrm{~mL})$ was added and the resulting mixture was allowed to stir at $-78{ }^{\circ} \mathrm{C}$ for $1 \mathrm{~h}$ at which time $\mathrm{Et}_{3} \mathrm{~N}(0.086 \mathrm{~mL}$, $0.60 \mathrm{mmol}, 50$ equiv) was added and the reaction mixture was allowed to warm to $0{ }^{\circ} \mathrm{C}$ over $1 \mathrm{~h}$. The reaction mixture was then quenched with saturated aqueous $\mathrm{NaCl}$

$(1 \mathrm{~mL})$ and extracted with $\mathrm{CH}_{2} \mathrm{Cl}_{2}(3 \times 1 \mathrm{~mL})$. The combined organic extracts were dried $\left(\mathrm{Na}_{2} \mathrm{SO}_{4}\right)$ and concentrated, and the obtained C-20-epi aldehyde 60 was used in the next step without further purification. C-20-epi aldehyde 60 (13.5 mg, $0.012 \mathrm{mmol}, 1.0$ equiv) was dissolved in $t-\mathrm{BuOH}_{\mathrm{H}} \mathrm{H}_{2} \mathrm{O}$ $(4: 1,1 \mathrm{~mL})$ at $25^{\circ} \mathrm{C}$, and to the solution was added $\mathrm{NaClO}_{2}\left(11 \mathrm{mg}, 0.12 \mathrm{mmol}, 10.0\right.$ equiv), $\mathrm{NaH}_{2} \mathrm{PO}_{4}$ (18 mg, $0.12 \mathrm{mmol}, 10.0$ equiv), and 2-methyl-2-butene $(0.2 \mathrm{~mL})$. The reaction mixture was allowed to stir at $25{ }^{\circ} \mathrm{C}$ for $1.5 \mathrm{~h}$, then quenched with a $\mathrm{pH} 7$ buffered saline solution $(3 \mathrm{~mL})$, and then extracted with EtOAc $(3 \times 2 \mathrm{~mL})$. The combined organic extracts were dried $\left(\mathrm{Na}_{2} \mathrm{SO}_{4}\right)$ and concentrated to afford crude C-20-epi carboxylic acid 62 which was used in the next step without further purification. C-20-epi carboxylic acid 62 (13 mg, $0.012 \mathrm{mmol}, 1.0$ equiv) was dissolved in THF (3 mL) and cooled to $0{ }^{\circ} \mathrm{C}$. TBAF $(0.060 \mathrm{~mL}, 1.0 \mathrm{M}$ in THF, $0.060 \mathrm{mmol})$ was added and the mixture was stirred for $2 \mathrm{~h}$ at $25^{\circ} \mathrm{C}$. The reaction mixture was quenched with saturated aqueous $\mathrm{NH}_{4} \mathrm{Cl}(5 \mathrm{~mL})$ and extracted with EtOAc (3 $\times 10 \mathrm{~mL})$. The combined organic extracts were washed with saturated aqueous $\mathrm{NaCl}(10 \mathrm{~mL})$, dried over $\mathrm{Na}_{2} \mathrm{SO}_{4}$, filtered, and concentrated. The crude product was purified by preparative TLC (silica gel, $\mathrm{CHCl}_{3}: \mathrm{MeOH}: \mathrm{H}_{2} \mathrm{O}$ 10:3:1) to afford C-20-epi free amine 64 (7.4 mg, $0.0084 \mathrm{mmol}, 70 \%$ over three steps) as a colorless oil. 64: $R_{\mathrm{f}}=0.36$ (silica gel, $\left.\mathrm{CHCl}_{3}: \mathrm{MeOH}: \mathrm{H}_{2} \mathrm{O} 20: 3: 1\right) ;[\alpha]_{\mathrm{D}}=-10.6(c=0.1$, $\mathrm{MeOH}$ ); IR (film) $v_{\max }=3383,2921,2850,1729,1713,1636,1455,1380,1094 \mathrm{~cm}^{-1} ;{ }^{1} \mathrm{H}$ NMR (500 $\left.\mathrm{MHz}, \mathrm{CD}_{3} \mathrm{OD}\right): \delta=6.01-5.98(\mathrm{~m}, 1 \mathrm{H}), 5.76-5.71(\mathrm{~m}, 1 \mathrm{H}), 5.67-5.65(\mathrm{~m}, 1 \mathrm{H}), 5.52(\mathrm{dd}, J=15.5,6.2$ 
Hz, $1 \mathrm{H}), 5.28(\mathrm{~d}, J=4.0 \mathrm{~Hz}, 1 \mathrm{H}), 5.14(\mathrm{~s}, 1 \mathrm{H}), 5.07$ (s, $1 \mathrm{H}), 4.94-4.91(\mathrm{~m}, 1 \mathrm{H}), 4.59-4.55(\mathrm{~m}, 1 \mathrm{H})$, $4.35(\mathrm{~d}, J=5.0 \mathrm{~Hz}, 1 \mathrm{H}), 4.34-4.32(\mathrm{~m}, 1 \mathrm{H}), 3.92-3.90(\mathrm{~m}, 1 \mathrm{H}), 3.63(\mathrm{~s}, 1 \mathrm{H}), 3.11-3.08(\mathrm{~m}, 2 \mathrm{H})$, 3.03-3.00 (m, $1 \mathrm{H}), 2.82(\mathrm{t}, J=12.0 \mathrm{~Hz}, 1 \mathrm{H}), 2.56(\mathrm{dd}, J=14.5,5.0 \mathrm{~Hz}, 1 \mathrm{H}), 2.37-2.35(\mathrm{~m}, 1 \mathrm{H})$, 2.33-2.17 (m, 6 H), $2.11(\mathrm{~s}, 3 \mathrm{H}), 2.09-1.99$ (m, $8 \mathrm{H}), 1.94-1.87$ (m, $4 \mathrm{H}), 1.94-1.87$ (m, $4 \mathrm{H}), 1.84-$ $1.74(\mathrm{~m}, 4 \mathrm{H}), 1.70-1.57(\mathrm{~m}, 3 \mathrm{H}), 1.55-1.48(\mathrm{~m}, 2 \mathrm{H}), 1.46-1.38(\mathrm{~m}, 2 \mathrm{H}), 1.05$ (d, J = 6.5 Hz, $3 \mathrm{H})$, $1.01(\mathrm{~d}, J=6.5 \mathrm{~Hz}, 3 \mathrm{H}), 0.93(\mathrm{~d}, J=6.5 \mathrm{~Hz}, 3 \mathrm{H}), 0.92(\mathrm{~d}, J=6.5 \mathrm{~Hz}, 3 \mathrm{H}), 0.89(\mathrm{~d}, J=6.5 \mathrm{~Hz}, 3 \mathrm{H})$; ${ }^{13} \mathrm{C} \mathrm{NMR}\left(125 \mathrm{MHz}, \mathrm{CDCl}_{3}\right): \delta=217.2,172.4,169.6,143.2,132.3,131.6,129.9,129.8,116.4,113.0$, $105.7,98.4,96.4,81.1,80.7,80.3,79.9,77.9,77.5,75.2,72.4,70.4,54.1,47.5,47.0,42.3,41.3,36.8$, $36.1,35.8,35.3,34.6,33.1,32.3,31.3,30.8,30.4,29.6,27.0,26.2,24.3,23.7,21.0,20.9,19.2,18.3$, 17.1, 15.6, 13.9; HRMS (MALDI-FTMS) for $\mathrm{C}_{49} \mathrm{H}_{73} \mathrm{NO}_{13} \mathrm{H}^{+}\left[\mathrm{M}+\mathrm{H}^{+}\right]$calcd 884.5154, found 884.5127.

C-20-FGHI-epi Free Amine 65. To a solution of oxalyl chloride ( $2 \mu \mathrm{L}, 0.12 \mathrm{mmol}, 10.0$ equiv) in $\mathrm{CH}_{2} \mathrm{Cl}_{2}(1 \mathrm{~mL})$ at $-78^{\circ} \mathrm{C}$ was added DMSO (15 $\mu \mathrm{L}, 0.24 \mathrm{mmol}, 20.0$ equiv). The solution was allowed

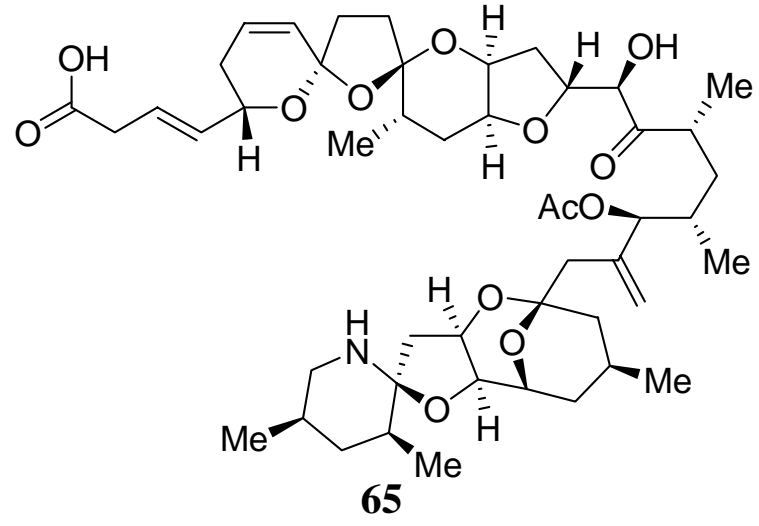
to stir for $20 \mathrm{~min}$ at $-78^{\circ} \mathrm{C}$ and then a solution of $\mathrm{C}-20$ FGHI-epi primary alcohol 59 (13.5 mg, $0.012 \mathrm{mmol}, 1.0$ equiv) in $\mathrm{CH}_{2} \mathrm{Cl}_{2}(0.5 \mathrm{~mL})$ was added. The resulting mixture was allowed to stir at $-78{ }^{\circ} \mathrm{C}$ for $1 \mathrm{~h}$ at which time $\mathrm{Et}_{3} \mathrm{~N}(0.086 \mathrm{~mL}, 0.60 \mathrm{mmol}, 50$ equiv) was added and the reaction mixture was allowed to warm to $0{ }^{\circ} \mathrm{C}$ over $1 \mathrm{~h}$. The reaction mixture was then quenched with saturated aqueous $\mathrm{NaCl}(1 \mathrm{~mL})$, extracted with $\mathrm{CH}_{2} \mathrm{Cl}_{2}(3 \times 1 \mathrm{~mL})$, dried $\left(\mathrm{Na}_{2} \mathrm{SO}_{4}\right)$ and concentrated. The obtained C-20-FGHI-epi aldehyde 61 was used in the next step without further purification. C-20FGHI-epi aldehyde 61 (13.5 mg, $0.012 \mathrm{mmol}, 1.0$ equiv) was dissolved in $t$ - $\mathrm{BuOH}: \mathrm{H}_{2} \mathrm{O}(4: 1,1 \mathrm{~mL})$ at $25^{\circ} \mathrm{C}$, and to the solution was added $\mathrm{NaClO}_{2}$ (11 mg, $0.12 \mathrm{mmol}, 10.0$ equiv), $\mathrm{NaH}_{2} \mathrm{PO}_{4}(18 \mathrm{mg}, 0.12$ mmol, 10.0 equiv), and 2-methyl-2-butene $(0.2 \mathrm{~mL})$. The reaction mixture was allowed to stir at $25^{\circ} \mathrm{C}$ for $1.5 \mathrm{~h}$, then quenched with a $\mathrm{pH} 7$ buffered saline solution $(3 \mathrm{~mL})$, and then extracted with EtOAc (3 $\times 2 \mathrm{~mL})$. The combined organic extracts were dried $\left(\mathrm{Na}_{2} \mathrm{SO}_{4}\right)$ and concentrated to afford crude C-20- 
FGHI-epi carboxylic acid 63, which was used in the next step without further purification. C-20-FGHIepi carboxylic acid 63 (13 mg, $0.012 \mathrm{mmol}, 1.0$ equiv) was dissolved in THF ( $3 \mathrm{~mL}$ ) and cooled to $0{ }^{\circ} \mathrm{C}$. TBAF $(0.060 \mathrm{~mL}, 1.0 \mathrm{M}$ in THF, $0.060 \mathrm{mmol})$ was added and the mixture was stirred for $2 \mathrm{~h}$ at $25^{\circ} \mathrm{C}$. The reaction was quenched with saturated aqueous $\mathrm{NH}_{4} \mathrm{Cl}(5 \mathrm{~mL})$ and extracted with EtOAc $(3 \times 10$ $\mathrm{mL})$. The combined organic extracts were washed with saturated aqueous $\mathrm{NaCl}(10 \mathrm{~mL})$, dried over $\mathrm{Na}_{2} \mathrm{SO}_{4}$, filtered, and concentrated, and the crude product was purified by preparative TLC (silica gel, $\mathrm{CHCl}_{3}: \mathrm{MeOH}: \mathrm{H}_{2} \mathrm{O}$ 10:3:1) to afford C-20-FGHI-epi free amine 65 (7.0 mg, $0.008 \mathrm{mmol}, 70 \%$ over three steps) as a colorless oil. 65: $R_{\mathrm{f}}=0.22$ (silica gel, $\left.\mathrm{CHCl}_{3}: \mathrm{MeOH}: \mathrm{H}_{2} \mathrm{O} 20: 3: 1\right) ;[\alpha]_{\mathrm{D}}=-57.7(c=$ 0.13, MeOH); IR (film) $v_{\max }=3438,2921,1731,1666,1643,1569,1449,1413,1239,1094 \mathrm{~cm}^{-1} ;{ }^{1} \mathrm{H}$ NMR $\left(500 \mathrm{MHz}, \mathrm{CD}_{3} \mathrm{OD}\right): \delta=6.04-6.01(\mathrm{~m}, 1 \mathrm{H}), 5.81-5.76(\mathrm{~m}, 1 \mathrm{H}), 5.70(\mathrm{dt}, J=10.0,2.0 \mathrm{~Hz}, 1 \mathrm{H})$, $5.54(\mathrm{dd}, J=15.8,6.2 \mathrm{~Hz}, 1 \mathrm{H}), 5.38$ (d, $J=4.0 \mathrm{~Hz}, 1 \mathrm{H}), 5.18$ (s, $1 \mathrm{H}), 5.09$ (s, $1 \mathrm{H}), 4.62-4.58$ (m, 1 H), 4.38-4.33 (m, 2 H), 4.29-4.24 (m, $1 \mathrm{H}), 3.95$ (br s, $1 \mathrm{H}), 3.76$ (d, J = 4.0 Hz, $1 \mathrm{H}), 3.71-3.64$ (m, 2 H), 3.15-3.11 (m, $1 \mathrm{H}), 2.82(\mathrm{t}, J=12.0 \mathrm{~Hz}, 1 \mathrm{H}), 2.56(\mathrm{dd}, J=14.5,5.0 \mathrm{~Hz}, 1 \mathrm{H}), 2.37-2.35(\mathrm{~m}, 1 \mathrm{H})$, 2.33-2.17 (m, 6 H), 2.11 (s, 3 H), 2.09-1.99 (m, 8 H), 1.94-1.87 (m, 4 H), 1.94-1.87 (m, 4 H), 1.841.74 (m, 4 H), 1.70-1.57 (m, 3 H), 1.55-1.48 (m, 2 H), 1.46-1.38 (m, 2 H), 1.05 (d, J = 6.5 Hz, 3 H), $1.01(\mathrm{~d}, J=6.5 \mathrm{~Hz}, 3 \mathrm{H}), 0.93$ (d, $J=6.5 \mathrm{~Hz}, 3 \mathrm{H}), 0.92$ (d, $J=6.5 \mathrm{~Hz}, 3 \mathrm{H}), 0.89$ (d, $J=6.5 \mathrm{~Hz}, 3 \mathrm{H})$; ${ }^{13} \mathrm{C} \mathrm{NMR}\left(125 \mathrm{MHz}, \mathrm{CDCl}_{3}\right): \delta=217.3,173.0,162.4,144.2,133.9,131.9,130.8,130.6,117.4,113.9$, $106.5,98.8,82.0,81.1,80.6,80.3,78.8,78.4,76.6,73.7,71.3,48.1,46.2,44.2,42.4,41.9,40.9,39.7$, $38.8,36.8,36.3,35.4,34.1,33.1,32.7,32.2,31.6,31.4,22.2,21.9,20.7,19.0,18.6,17.8,17.1,17.0$; HRMS (MALDI-FTMS) for $\mathrm{C}_{49} \mathrm{H}_{73} \mathrm{NO}_{13} \mathrm{H}^{+}\left[\mathrm{M}+\mathrm{H}^{+}\right]$calcd 884.5154, found 884.5145. 
C-20-epi Azaspiracid-1 Structure 2. To a solution of C-20-epi free amine 64 (5.0 $\mathrm{mg}, 0.0056 \mathrm{mmol})$ in $\mathrm{MeOH}: \mathrm{H}_{2} \mathrm{O}(5: 1,1 \mathrm{~mL})$ at $25{ }^{\circ} \mathrm{C}$ was added a solution of $\mathrm{LiOH}\left(56 \mu \mathrm{L}, 0.056 \mathrm{mmol}, 1.0 \mathrm{M}\right.$ in $\mathrm{H}_{2} \mathrm{O}, 10$

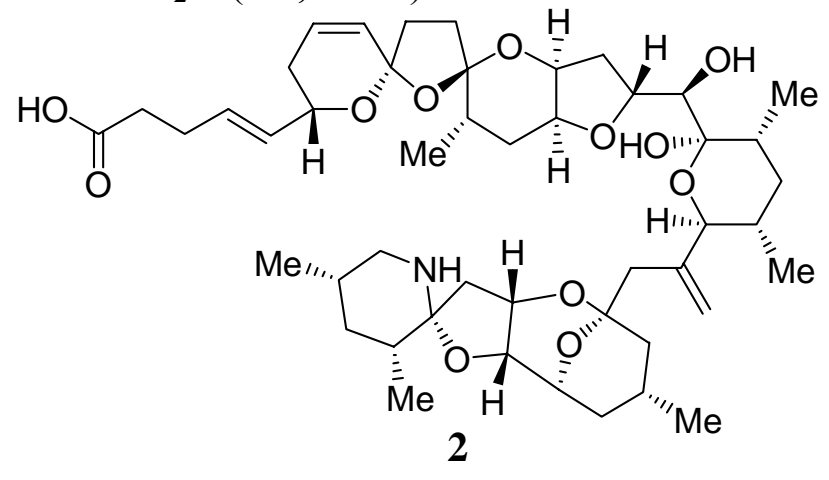
equiv). The reaction mixture was allowed to stir for 16 $\mathrm{h}$ at which time the starting material was consumed (TLC). The reaction mixture was then directly purified by preparative TLC (silica gel, $\mathrm{CHCl}_{3}: \mathrm{MeOH}: \mathrm{H}_{2} \mathrm{O}$ $20: 3: 1)$ to afford what is believed to be C-20-epi originally proposed azaspiracid-1 structure 2 as a colorless oil (2.1 mg, $0.0025 \mathrm{mmol}, 45 \%)$ existing as an inseparable mixture of compounds. Further purification by HPLC using the conditions described in the isolation of azaspiracid- $1^{1}$ failed to yield a pure sample. 2: $R_{\mathrm{f}}=0.40$ (silica gel, $\mathrm{CHCl}_{3}: \mathrm{MeOH}: \mathrm{H}_{2} \mathrm{O}$ 20:3:1); HRMS (MALDI-FTMS) for $\mathrm{C}_{47} \mathrm{H}_{71} \mathrm{NO}_{12} \mathrm{H}^{+}\left[\mathrm{M}+\mathrm{H}^{+}\right]$calcd 842.5049, found 842.5044.

C-20-FGHI-epi Azaspiracid-1 Structure 3. To a solution of C-20-FGHI-epi free amine 65 (4.5 mg, $0.0050 \mathrm{mmol})$ in $\mathrm{MeOH}: \mathrm{H}_{2} \mathrm{O}(5: 1,1 \mathrm{~mL})$ at $25{ }^{\circ} \mathrm{C}$ was added a solution of $\mathrm{LiOH}(50 \mu \mathrm{L}, 0.050 \mathrm{mmol}$, (n) $1.0 \mathrm{M}$ in $\mathrm{H}_{2} \mathrm{O}, 10$ equiv). The reaction mixture was allowed to stir for $16 \mathrm{~h}$ at which time the starting material was consumed (TLC). The reaction mixture was then purified by preparative TLC (silica gel, $\left.\mathrm{CHCl}_{3}: \mathrm{MeOH}: \mathrm{H}_{2} \mathrm{O} 20: 3: 1\right)$ to afford what is believed to be originally proposed C-20-FGHI-epi azaspiracid1 structure 3 as a colorless oil (1.6 $\mathrm{mg}, 0.0019 \mathrm{mmol}, 38 \%)$ existing as an inseparable mixture of compounds. Further purification by HPLC using the conditions described in the isolation of azaspiracid$1^{1}$ failed to yield a pure sample. 3: $R_{\mathrm{f}}=0.34$ (silica gel, $\mathrm{CHCl}_{3}: \mathrm{MeOH}: \mathrm{H}_{2} \mathrm{O}$ 20:3:1); HRMS (MALDIFTMS) for $\mathrm{C}_{47} \mathrm{H}_{71} \mathrm{NO}_{12} \mathrm{H}^{+}\left[\mathrm{M}+\mathrm{H}^{+}\right]$calcd 842.5049, found 842.5051. 
Diacetate 66. To a solution of tetraol $28\left(280 \mathrm{mg}, 0.427 \mathrm{mmol}, 1.0\right.$ equiv) in $\mathrm{CH}_{2} \mathrm{Cl}_{2}(50 \mathrm{~mL})$ at $-78{ }^{\circ} \mathrm{C}$ was added 2,4,6-collidine $(2.27 \mathrm{~mL}, 17.1 \mathrm{mmol}, 40$ equiv) followed by the dropwise addition of $\mathrm{AcCl}$

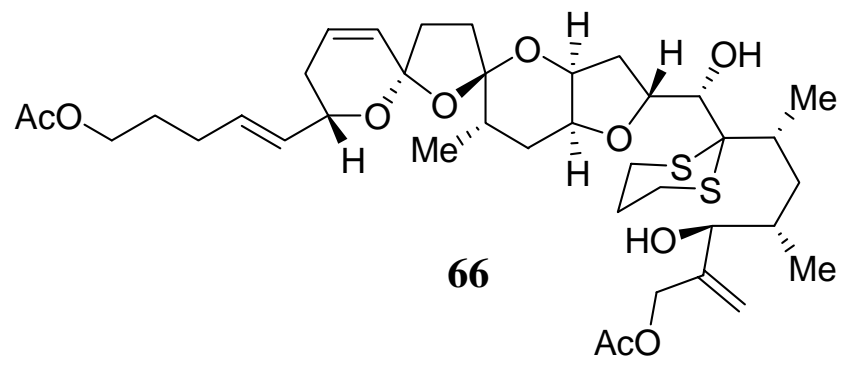
(0.610 $\mathrm{mL}, 8.54 \mathrm{mmol}, 20$ equiv). The reaction mixture was stirred at $-78^{\circ} \mathrm{C}$ for $5 \mathrm{~h}$ and was then quenched with $\mathrm{H}_{2} \mathrm{O}(20 \mathrm{~mL})$. The resulting biphasic mixture was extracted with $\mathrm{CH}_{2} \mathrm{Cl}_{2}(3 \times 20 \mathrm{~mL})$ and the combined organic layers were dried $\left(\mathrm{Na}_{2} \mathrm{SO}_{4}\right)$, concentrated, and then purified by flash column chromatography (silica gel, hexanes:EtOAc 10:1 $\rightarrow$ 1:1) to provide diacetate 66 (280 $\mathrm{mg}, 0.379 \mathrm{mmol}, 89 \%$ ) as a colorless solid. 66: $R_{\mathrm{f}}=0.40$ (silica gel, EtOAc:hexanes 2:1); $[\alpha]_{\mathrm{D}}^{33}=-39.8\left(\mathrm{CH}_{3} \mathrm{OH}, c=0.40\right)$; IR (film) $v_{\max }=3468,2957,2929,1738,1458$, 1367, 1238, 1094, $1044 \mathrm{~cm}^{-1} ;{ }^{1} \mathrm{H}$ NMR (600 MHz, $\left.\mathrm{CD}_{2} \mathrm{Cl}_{2}\right): \delta=5.97-5.92(\mathrm{~m}, 1 \mathrm{H}), 5.68-5.64(\mathrm{~m}, 1$ H), 5.63-5.60 (m, $1 \mathrm{H}), 5.49(\mathrm{dd}, J=15.8,6.1 \mathrm{~Hz}, 1 \mathrm{H}), 5.04(\mathrm{~s}, 2 \mathrm{H}), 4.95-4.91(\mathrm{~m}, 1 \mathrm{H}), 4.61(\mathrm{dd}, J=$ 21.0, 14.0 Hz, 2 H), 4.38-4.33 (m, $1 \mathrm{H}), 3.36$ (br s, $1 \mathrm{H}), 4.01$ (t, J=6.5 Hz, $2 \mathrm{H}), 3.95$ (br s, 2 H), 3.86 (d, $J=6.6 \mathrm{~Hz}, 1 \mathrm{H}), 3.36$ (br s, $1 \mathrm{H}), 3.22$ (d, $J=4.4 \mathrm{~Hz}, 1 \mathrm{H}), 3.03$ (ddd, $J=14.5,10.9,3.5 \mathrm{~Hz}, 1 \mathrm{H})$, $2.85(\mathrm{ddd}, J=14.5,10.9,3.5 \mathrm{~Hz}, 1 \mathrm{H}), 2.66(\mathrm{dt}, J=14.5,4.4 \mathrm{~Hz}, 1 \mathrm{H}), 2.61(\mathrm{dt}, J=14.5,4.4 \mathrm{~Hz}, 1 \mathrm{H})$, 2.44-2.40 (m, 1H), 2.35-2.29 (m, $1 \mathrm{H}), 2.18(\mathrm{dd}, J=12.7,5.6 \mathrm{~Hz}, 1 \mathrm{H}), 2.13-1.86$ (m, $18 \mathrm{H}), 1.83-1.77$ $(\mathrm{m}, 1 \mathrm{H}), 1.71-1.66(\mathrm{~m}, 3 \mathrm{H}), 1.45(\mathrm{dt}, J=13.1,2.6 \mathrm{~Hz}, 1 \mathrm{H}), 1.26(\mathrm{~d}, J=6.6 \mathrm{~Hz}, 3 \mathrm{H}), 1.13$ (ddd, $J=$ 14.0, 10.6, 3.1 Hz, $1 \mathrm{H}), 0.88(\mathrm{~d}, J=7.0 \mathrm{~Hz}, 3 \mathrm{H}), 0.80(\mathrm{~d}, J=6.6 \mathrm{~Hz}, 3 \mathrm{H}) ;{ }^{13} \mathrm{C} \mathrm{NMR}(150 \mathrm{MHz}$, $\left.\mathrm{CD}_{2} \mathrm{Cl}_{2}\right): \delta=171.2,170.8,146.1,131.3,131.2,129.4,128.7,113.2,111.7,104.4,82.0,77.9,77.1,76.1$, $75.0,69.1,64.0,63.6,63.5,40.4,40.2,39.3,36.2,35.5,34.3,31.6,30.5,29.0,28.5,26.9,25.9,25.0$, 24.0, 21.1, 21.0, 19.7, 17.1, 15.7; HR-MS [electrospray ionization (ESI)]: calcd for $\mathrm{C}_{38} \mathrm{H}_{59} \mathrm{O}_{10} \mathrm{~S}_{2} \mathrm{Na}^{+}[\mathrm{M}$ $\left.+\mathrm{H}^{+}\right]:$739.3544, found 739.3530 .

TBS ether 67. To a solution of diacetate 66 (315 mg, $0.427 \mathrm{mmol}, 1.0$ equiv) in $\mathrm{CH}_{2} \mathrm{Cl}_{2}(50 \mathrm{~mL})$ at -78 ${ }^{\circ} \mathrm{C}$ was added 2,6-lutidine $(0.994 \mathrm{~mL}, 8.54 \mathrm{mmol}, 20$ equiv) followed by the dropwise addition of TBSOTf ( $0.980 \mathrm{~mL}, 4.27 \mathrm{mmol}, 10$ equiv). The solution was warmed to $0{ }^{\circ} \mathrm{C}$ over $30 \mathrm{~min}$ and was then quenched with saturated aqueous $\mathrm{NaHCO}_{3}(30 \mathrm{~mL})$. The resulting biphasic mixture was then extracted 
with $\mathrm{CH}_{2} \mathrm{Cl}_{2}(3 \times 20 \mathrm{~mL})$ and the combined organic extracts were dried $\left(\mathrm{Na}_{2} \mathrm{SO}_{4}\right)$, concentrated, and then purified by flash column chromatography (silica gel, EtOAc:hexanes 1:2) to give TBS ether 67

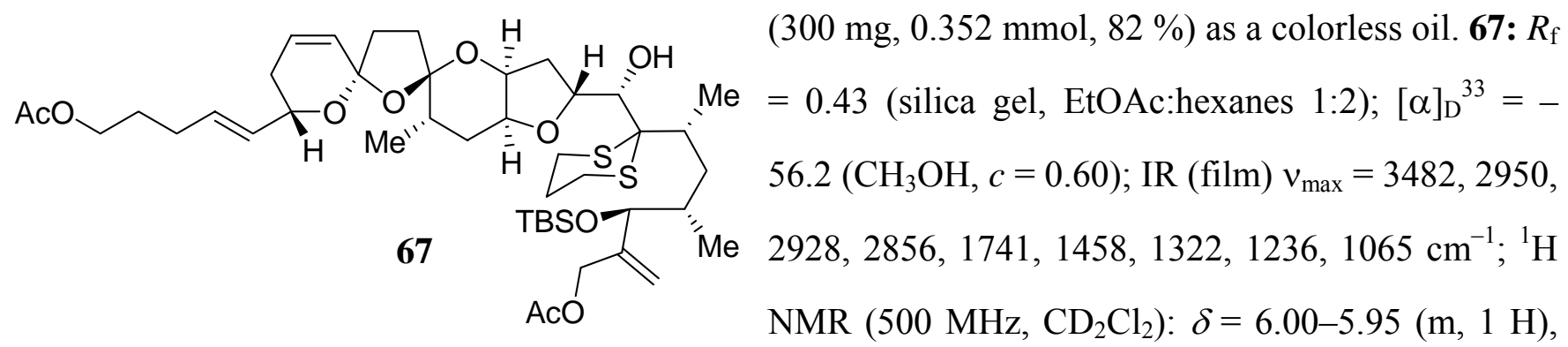
5.69-5.62 (m, $2 \mathrm{H}), 5.50(\mathrm{dd}, J=15.8,6.3 \mathrm{~Hz}, 1 \mathrm{H}), 5.14(\mathrm{~s}, 2 \mathrm{H}), 4.83(\mathrm{dd}, J=9.2,7.0 \mathrm{~Hz}, 1 \mathrm{H}), 4.58$ (s, 2 H), 4.40-4.36 (m, $1 \mathrm{H}), 4.15$ (br s, $1 \mathrm{H}), 4.02$ (t, J = 6.6 Hz, 2 H), 3.92 (d, J = 5.9 Hz, $1 \mathrm{H}), 3.90$ (br s, $1 \mathrm{H}), 3.59(\mathrm{~d}, J=9.9 \mathrm{~Hz}, 1 \mathrm{H}), 3.21-3.15(\mathrm{~m}, 1 \mathrm{H}), 2.99(\mathrm{~d}, J=10.3 \mathrm{~Hz}, 1 \mathrm{H}), 2.84-2.79(\mathrm{~m}, 1 \mathrm{H})$, 2.76-2.71 (m, $1 \mathrm{H}), 2.58-2.50(\mathrm{~m}, 1 \mathrm{H}), 2.30$ (d, $J=14.3 \mathrm{~Hz}, 1 \mathrm{H}), 2.14-1.86(\mathrm{~m}, 20 \mathrm{H}), 1.87-1.77(\mathrm{~m}$, $1 \mathrm{H}), 1.69(\mathrm{~m}, 3 \mathrm{H}), 1.51-1.46(\mathrm{~m}, 1 \mathrm{H}), 1.22(\mathrm{~d}, J=7.0 \mathrm{~Hz}, 3 \mathrm{H}), 0.91(\mathrm{~s}, 9 \mathrm{H}), 0.88(\mathrm{~d}, J=6.3 \mathrm{~Hz}, 3$ $\mathrm{H}), 0.87(\mathrm{~d}, J=6.3 \mathrm{~Hz}, 3 \mathrm{H}), 0.83-0.76(\mathrm{~m}, 1 \mathrm{H}), 0.07(\mathrm{~s}, 3 \mathrm{H}), 0.00(\mathrm{~s}, 3 \mathrm{H}) ;{ }^{13} \mathrm{C} \mathrm{NMR}(125 \mathrm{MHz}$, $\left.\mathrm{CD}_{2} \mathrm{Cl}_{2}\right): \delta=171.2,170.8,145.6,131.4,131.1,129.6,128.6,113.9,111.7,104.4,79.4,77.7,77.5,76.3$, $76.1,69.1,64.0,64.0,63.1,40.3,39.6,38.7,36.2,35.4,34.4,31.5,30.5,29.0,28.5,27.5,26.7,26.1$, 24.9, 23.7, 21.1, 18.8, 18.4, 17.3, 15.8, -4.5, -4.8; HR-MS [electrospray ionization (ESI)]: calcd for $\mathrm{C}_{44} \mathrm{H}_{72} \mathrm{O}_{10} \mathrm{~S}_{2} \mathrm{SiH}^{+}\left[\mathrm{M}+\mathrm{H}^{+}\right]: 853.4409$, found 853.4395 .

Ketone 68. To a solution of TBS ether 67 (200 mg, $0.214 \mathrm{mmol}, 1.0$ equiv) in $\mathrm{CH}_{3} \mathrm{CN}(140 \mathrm{~mL})$ and $\mathrm{pH}$ 7 buffered saline solution $(35 \mathrm{~mL})$ at $-5{ }^{\circ} \mathrm{C}$ was added a solution of $\mathrm{PhI}\left(\mathrm{OCOCF}_{3}\right)_{2}(8.56 \mathrm{~mL}, 0.05 \mathrm{M}$ in

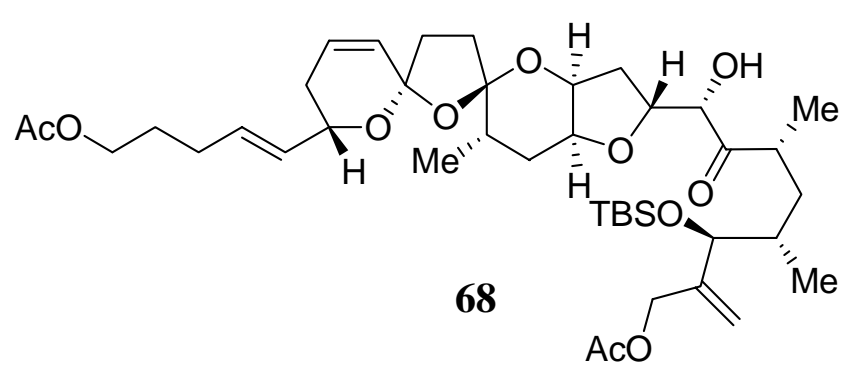
$\mathrm{CH}_{3} \mathrm{CN}, 0.428 \mathrm{mmol}, 2$ equiv). The reaction mixture was stirred at $-5{ }^{\circ} \mathrm{C}$ for $30 \mathrm{~min}$, then quenched with saturated aqueous $\mathrm{Na}_{2} \mathrm{~S}_{2} \mathrm{O}_{3}(100 \mathrm{~mL})$ and extracted with EtOAc $(3 \times 50 \mathrm{~mL})$. The combined organic extracts were dried $\left(\mathrm{Na}_{2} \mathrm{SO}_{4}\right)$, concentrated, and then purified by flash column chromatography (hexanes:EtOAc 10:1 $\rightarrow$ 1:1) to afford ketone 68 (100 mg, $0.131 \mathrm{mmol}, 61 \%$ ) as a colorless oil. 68: $R_{\mathrm{f}}=0.18$ (silica gel, EtOAc:hexanes 1:2); $[\alpha]_{\mathrm{D}}{ }^{33}=-16.5$ 
$\left(\mathrm{CH}_{3} \mathrm{OH}, c=0.2\right)$; IR (film) $v_{\max }=3466,2956,2929,2856,1741,1459,1366,1322,1248,1071 \mathrm{~cm}^{-1}$; ${ }^{1} \mathrm{H}$ NMR (600 MHz, $\left.\mathrm{CD}_{2} \mathrm{Cl}_{2}\right): \delta=6.00-5.93(\mathrm{~m}, 1 \mathrm{H}), 5.72-5.61(\mathrm{~m}, 2 \mathrm{H}), 5.49(\mathrm{dd}, J=15.4,5.7 \mathrm{~Hz}, 1$ H), $5.16(\mathrm{~d}, J=4.9 \mathrm{~Hz}, 2 \mathrm{H}), 4.78(\mathrm{dd}, J=8.3,6.2 \mathrm{~Hz}, 1 \mathrm{H}), 4.58(\mathrm{~d}, J=14.0 \mathrm{~Hz}, 1 \mathrm{H}), 4.48(\mathrm{~d}, J=14.0$ $\mathrm{Hz}, 1 \mathrm{H}), 4.36(\mathrm{~m}, 1 \mathrm{H}), 4.22(\mathrm{~d}, J=5.7 \mathrm{~Hz}, 1 \mathrm{H}), 4.18($ br s, $1 \mathrm{H}), 4.01(\mathrm{t}, J=7.0 \mathrm{~Hz}, 2 \mathrm{H}), 3.93(\mathrm{~d}, J=$ $5.3 \mathrm{~Hz}, 1 \mathrm{H}), 3.81$ (br s, $1 \mathrm{H}), 3.57$ (d, J=5.7 Hz, $1 \mathrm{H}), 3.08-2.99$ (m, $1 \mathrm{H}), 2.43-2.36$ (m, $1 \mathrm{H}), 2.20-$ $2.14(\mathrm{~m}, 1 \mathrm{H}), 2.12-1.96(\mathrm{~m}, 17 \mathrm{H}), 1.74-1.65$ (m, $2 \mathrm{H}), 1.63-1.55$ (m, $1 \mathrm{H}), 1.39-1.30$ (m, $1 \mathrm{H}), 1.09$ $(\mathrm{d}, J=7.0 \mathrm{~Hz}, 3 \mathrm{H}), 0.93(\mathrm{~s}, 9 \mathrm{H}), 0.98-0.90(\mathrm{~m}, 1 \mathrm{H}), 0.87(\mathrm{~d}, J=6.6 \mathrm{~Hz}, 3 \mathrm{H}), 0.83$ (d, J = 7.0 Hz, 3 $\mathrm{H}), 0.06(\mathrm{~s}, 3 \mathrm{H}), 0.00(\mathrm{~s}, 3 \mathrm{H}) ;{ }^{13} \mathrm{C} \mathrm{NMR}\left(125 \mathrm{MHz}, \mathrm{CD}_{2} \mathrm{Cl}_{2}\right): \delta=213.4,171.2,170.7,145.7,131.4$, $131.1,129.6,128.6,113.2,111.7,104.4,78.9,78.8,77.7,76.4,76.1,69.0,64.3,64.0,39.1,36.3,36.1$, $34.8,34.2,34.1,31.5,30.5,29.0,28.5,26.0,23.7,21.1,19.9,18.5,17.1,15.4,-4.5,-5.0$; HR-MS [electrospray ionization (ESI)]: calcd for $\mathrm{C}_{41} \mathrm{H}_{66} \mathrm{O}_{11} \mathrm{SiH}^{+}\left[\mathrm{M}+\mathrm{H}^{+}\right]$: 763.4447, found 763.4433.

Stille coupling product 69. Ketone 68 (50 mg, $0.072 \mathrm{mmol}, 1.0$ equiv) was azeotroped with benzene (3

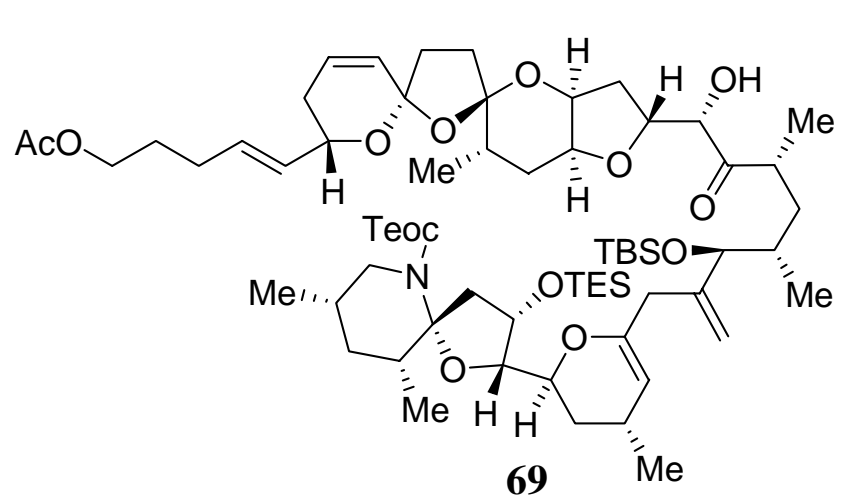
$\times 5 \mathrm{~mL})$ and then dissolved in NMP $(2.5 \mathrm{~mL})$ at 25 ${ }^{\circ} \mathrm{C}$. $\mathrm{LiCl}$ (19 mg, $0.44 \mathrm{mmol}, 6.0$ equiv), $i-\operatorname{Pr}_{2} \mathrm{NEt}$ (0.075 mL, $0.44 \mathrm{mmol}, 6.0$ equiv) and $\mathrm{AsPh}_{3}(7 \mathrm{mg}$, $0.022 \mathrm{mmol}, 0.3$ equiv) were added at $25^{\circ} \mathrm{C}$ followed by $\mathrm{Pd}_{2} \mathrm{dba}_{3}(20 \mathrm{mg}, 0.022 \mathrm{mmol}, 0.3$ equiv). The reaction mixture was heated to $45^{\circ} \mathrm{C}$ and to this solution was added a solution of vinyl stannane 6 (153 mg, $0.22 \mathrm{mmol}, 3.0$ equiv) in THF (2.5 mL) over a period of $4 \mathrm{~h}$ via syringe pump. The reaction mixture was cooled to $25{ }^{\circ} \mathrm{C}$ and quenched slowly with saturated aqueous $\mathrm{NaHCO}_{3}(2 \mathrm{~mL})$. The aqueous layer was extracted with EtOAc $(3 \times 10 \mathrm{~mL})$ and the combined organic layers were washed with brine $(10 \mathrm{~mL})$, dried over $\mathrm{Na}_{2} \mathrm{SO}_{4}$, and concentrated. The crude product was purified by flash column chromatography (silica gel, hexanes:EtOAc 3:1) to afford Stille coupling product 69 (55 mg, $0.045 \mathrm{mmol}, 63 \%$ ) as a colorless oil. 69: $R_{\mathrm{f}}=0.58$ (silica gel, EtOAc:hexanes $\left.1: 1\right) ;[\alpha]_{\mathrm{D}}{ }^{33}=+11.4$ 
$\left(\mathrm{CH}_{3} \mathrm{OH}, \mathrm{c}=0.7\right)$; IR (film) $v_{\max }=3461,2955,2929,2874,1742,1694,1455,1393,1360,1250,1172$ $\mathrm{cm}^{-1} ;{ }^{1} \mathrm{H}$ NMR $\left(600 \mathrm{MHz}, \mathrm{CD}_{2} \mathrm{Cl}_{2}\right): \delta=6.03-5.96(\mathrm{~m}, 1 \mathrm{H}), 5.72-5.67(\mathrm{~m}, 1 \mathrm{H}), 5.66-5.64(\mathrm{~m}, 1 \mathrm{H})$, $5.49(\mathrm{dd}, J=15.3,6.1 \mathrm{~Hz}, 1 \mathrm{H}), 5.05(\mathrm{~s}, 1 \mathrm{H}), 5.03(\mathrm{~s}, 1 \mathrm{H}), 4.79(\mathrm{dd}, J=8.3,7.4 \mathrm{~Hz}, 1 \mathrm{H}), 4.57-4.53$ $(\mathrm{m}, 1 \mathrm{H}), 4.51(\mathrm{~d}, J=5.3 \mathrm{~Hz}, 1 \mathrm{H}), 4.39-4.30(\mathrm{~m}, 1 \mathrm{H}), 4.21(\mathrm{~d}, J=5.7 \mathrm{~Hz}, 1 \mathrm{H}), 4.18(\mathrm{br} \mathrm{s}, 1 \mathrm{H}), 4.15$ $(\mathrm{dd}, J=6.6,4.4 \mathrm{~Hz}, 1 \mathrm{H}), 4.11-4.07(\mathrm{~m}, 2 \mathrm{H}), 4.06-4.02(\mathrm{~m}, 1 \mathrm{H}), 4.01(\mathrm{t}, J=7.0 \mathrm{~Hz}, 2 \mathrm{H}), 3.92(\mathrm{~d}, J=$ $3.0 \mathrm{~Hz}, 1 \mathrm{H}), 3.82$ (br s, $1 \mathrm{H}), 3.67$ (dd, $J=13.3,2.6 \mathrm{~Hz}, 1 \mathrm{H}), 3.58$ (d, $J=5.7 \mathrm{~Hz}, 1 \mathrm{H}), 3.07$ (t, $J=11.9$ Hz, $1 \mathrm{H}), 3.04-2.94(\mathrm{~m}, 1 \mathrm{H}), 2.90$ (dd, $J=14.5,6.6 \mathrm{~Hz}, 1 \mathrm{H}), 2.84$ (d, $J=15.8 \mathrm{~Hz}, 1 \mathrm{H}), 2.57$ (d, $J=$ $15.8 \mathrm{~Hz}, 1 \mathrm{H}), 2.48(\mathrm{dd}, J=14.5,7.0 \mathrm{~Hz}, 1 \mathrm{H}), 2.44-2.35$ (m, $1 \mathrm{H}), 2.25$ (br s, $1 \mathrm{H}), 2.11-1.80$ (m, 17 H), 1.72-1.65 (m, 2 H), 1.64-1.50 (m, $3 \mathrm{H}), 1.50-1.40(\mathrm{~m}, 1 \mathrm{H}), 1.39-1.34(\mathrm{~m}, 1 \mathrm{H}), 1.33-1.28$ (m, 1 H), $1.08(\mathrm{~d}, J=7.4 \mathrm{~Hz}, 3 \mathrm{H}), 1.01-0.97(\mathrm{~m}, 5 \mathrm{H}), 0.97-0.90(\mathrm{~m}, 19 \mathrm{H}), 0.88(\mathrm{~d}, J=6.6 \mathrm{~Hz}, 3 \mathrm{H}), 0.84$ $(\mathrm{d}, J=7.0 \mathrm{~Hz}, 3 \mathrm{H}), 0.81(\mathrm{~d}, J=6.1 \mathrm{~Hz}, 3 \mathrm{H}), 0.80(\mathrm{~d}, J=6.5 \mathrm{~Hz}, 3 \mathrm{H}), 0.60(\mathrm{t}, J=7.9 \mathrm{~Hz}, 6 \mathrm{H}), 0.04(\mathrm{~s}$, $9 \mathrm{H}), 0.03(\mathrm{~s}, 3 \mathrm{H}), 0.00(\mathrm{~s}, 3 \mathrm{H}) ;{ }^{13} \mathrm{C} \mathrm{NMR}\left(150 \mathrm{MHz}, \mathrm{CD}_{2} \mathrm{Cl}_{2}\right): \delta=213.5,171.1,156.4,151.5,147.7$, $131.3,131.1,129.6,128.6,112.0,111.7,104.4,104.3,96.6,83.5,79.0,78.9,77.6,76.4,76.0,72.5,71.8$, 69.0, 64.0, 63.1, 49.4, 43.6, 39.2, 38.6, 38.5, 38.1, 36.4, 36.1, 34.3, 33.7, 32.9, 31.5, 31.4, 31.0, 30.5, $29.0,28.5,26.1,25.0,23.7,23.2,21.1,19.9,18.7,18.5,17.9,17.7,16.9,15.7,7.0,5.0,-1.5,-4.2,-4.8$; HR-MS [electrospray ionization (ESI)]: calcd for $\mathrm{C}_{67} \mathrm{H}_{115} \mathrm{NO}_{14} \mathrm{Si}_{3} \mathrm{Na}^{+}\left[\mathrm{M}+\mathrm{Na}^{+}\right]$: 1264.7517, found 1264.7495.

Diol 70. To a solution of Stille coupling product $69(20 \mathrm{mg}, 0.016 \mathrm{mmol}, 1.0$ equiv) in THF (2 mL) at 0

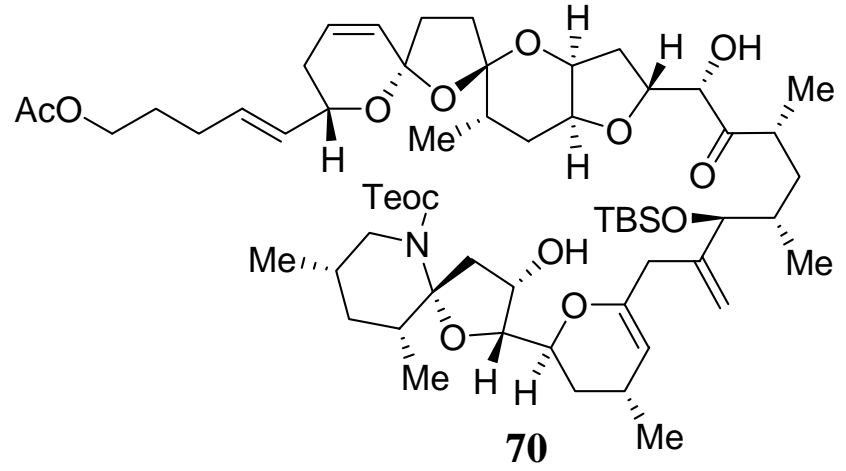
${ }^{\circ} \mathrm{C}$ was added TBAF $(24 \mu \mathrm{L}, 1.0 \mathrm{M}$ in THF, 0.024 mmol, 1.5 equiv). The reaction mixture was stirred at $0{ }^{\circ} \mathrm{C}$ for $10 \mathrm{~min}$, then concentrated and purified by flash column chromatography (silica gel, EtOAc:hexanes 1:2) to give diol $70(16 \mathrm{mg}, 0.014$ mmol, 88\%) as a colorless oil. 70: $R_{\mathrm{f}}=0.63$ (silica gel, EtOAc:hexanes 1:1); $[\alpha]_{\mathrm{D}}^{33}=-5.0\left(\mathrm{CH}_{3} \mathrm{OH}, c=0.4\right)$; IR (film) $v_{\max }=3462,2956,2926,2852$, 
$1741,1701,1676,1459,1396,1250,1173,1070 \mathrm{~cm}^{-1} ;{ }^{1} \mathrm{H} \mathrm{NMR}\left(600 \mathrm{MHz}, \mathrm{CD}_{2} \mathrm{Cl}_{2}\right): \delta=6.02-5.96(\mathrm{~m}$, $1 \mathrm{H}), 5.72-5.69$ (m, $1 \mathrm{H}), 5.68-5.62(\mathrm{~m}, 1 \mathrm{H}), 5.50(\mathrm{dd}, J=15.4,6.2 \mathrm{~Hz}, 1 \mathrm{H}), 5.04(\mathrm{~s}, 1 \mathrm{H}), 4.96(\mathrm{~s}, 1$ H), $4.77(\mathrm{dd}, J=8.3,7.0 \mathrm{~Hz}, 1 \mathrm{H}), 4.58(\mathrm{~d}, J=4.0 \mathrm{~Hz}, 1 \mathrm{H}), 4.45-4.40(\mathrm{~m}, 1 \mathrm{H}), 4.39-4.31(\mathrm{~m}, 1 \mathrm{H})$, $4.20(\mathrm{~d}, J=6.1 \mathrm{~Hz}, 1 \mathrm{H}), 4.20-4.13(\mathrm{~m}, 2 \mathrm{H}), 4.11(\mathrm{t}, J=8.8 \mathrm{~Hz}, 2 \mathrm{H}), 4.02(\mathrm{t}, J=6.5 \mathrm{~Hz}, 2 \mathrm{H}), 3.92-$ $3.85(\mathrm{~m}, 2 \mathrm{H}), 3.81$ (br s, $1 \mathrm{H}), 3.74-3.65(\mathrm{~m}, 1 \mathrm{H}), 3.62(\mathrm{~d}, J=5.7 \mathrm{~Hz}, 1 \mathrm{H}), 3.27$ (dd, J=14.5, 3.1 Hz, $1 \mathrm{H}), 3.22(\mathrm{~d}, J=5.7 \mathrm{~Hz}, 1 \mathrm{H}), 3.05-2.98(\mathrm{~m}, 2 \mathrm{H}), 2.78(\mathrm{~d}, J=15.8 \mathrm{~Hz}, 1 \mathrm{H}), 2.52(\mathrm{~d}, J=15.8 \mathrm{~Hz}, 1$ H), 2.44-2.36 (m, 2 H), 2.29 (br s, $1 \mathrm{H}), 2.12-1.80$ (m, $17 \mathrm{H}), 1.75-1.72(\mathrm{~m}, 1 \mathrm{H}), 1.71-1.64(\mathrm{~m}, 2 \mathrm{H})$, 1.61-1.51 (m, $3 \mathrm{H}), 1.42-1.32(\mathrm{~m}, 1 \mathrm{H}), 1.30-1.22(\mathrm{~m}, 1 \mathrm{H}), 1.08(\mathrm{~d}, J=7.0 \mathrm{~Hz}, 3 \mathrm{H}), 1.03(\mathrm{~d}, J=7.0$ Hz, 2 H), 1.03-0.96 (m, 2 H), 0.93 (br s, $10 \mathrm{H}), 0.86$ (d, J=7.0 Hz, $3 \mathrm{H}), 0.85$ (d, J = 6.5 Hz, $3 \mathrm{H}), 0.84$ $(\mathrm{d}, J=7.0 \mathrm{~Hz}, 3 \mathrm{H}), 0.82(\mathrm{~d}, J=6.6 \mathrm{~Hz}, 3 \mathrm{H}), 0.04(\mathrm{~s}, 12 \mathrm{H}), 0.00(\mathrm{~s}, 3 \mathrm{H}) ;{ }^{13} \mathrm{C} \mathrm{NMR}(125 \mathrm{MHz}$, $\left.\mathrm{CD}_{2} \mathrm{Cl}_{2}\right): \delta=213.6,171.2,156.8,150.4,147.8,131.4,131.1,129.6,128.6,111.7,105.3,104.4,97.5$, 83.6, 79.8, 78.9, 77.7, 76.4, 76.2, 72.7, 71.0, 69.0, 64.1, 63.5, 49.7, 40.5, 39.3, 38.9, 37.8, 37.7, 36.4, $36.2,34.3,34.1,33.6,32.5,31.5,31.3,30.5,29.0,28.5,26.2,24.5,23.7,22.7,21.1,19.9,18.9,18.5$, 17.9, 17.6, 16.9, 15.7, -1.4, -4.2, -4.9; HR-MS [electrospray ionization (ESI)]: calcd for $\mathrm{C}_{61} \mathrm{H}_{101} \mathrm{NO}_{14} \mathrm{Si}_{2} \mathrm{Na}^{+}\left[\mathrm{M}+\mathrm{Na}^{+}\right]: 1150.6652$, found 1150.6639 .

Iodoetherification product 71. To a solution of diol 70 (18 mg, $16 \mu \mathrm{mol}, 1.0$ equiv) in THF (2 mL) at 0 ${ }^{\circ} \mathrm{C}$ was added $\mathrm{NaHCO}_{3}(13 \mathrm{mg}, 160 \mu \mathrm{mol}, 10$ equiv) followed by NIS (7.2 mg, $32 \mu \mathrm{mol}, 2$ equiv). The

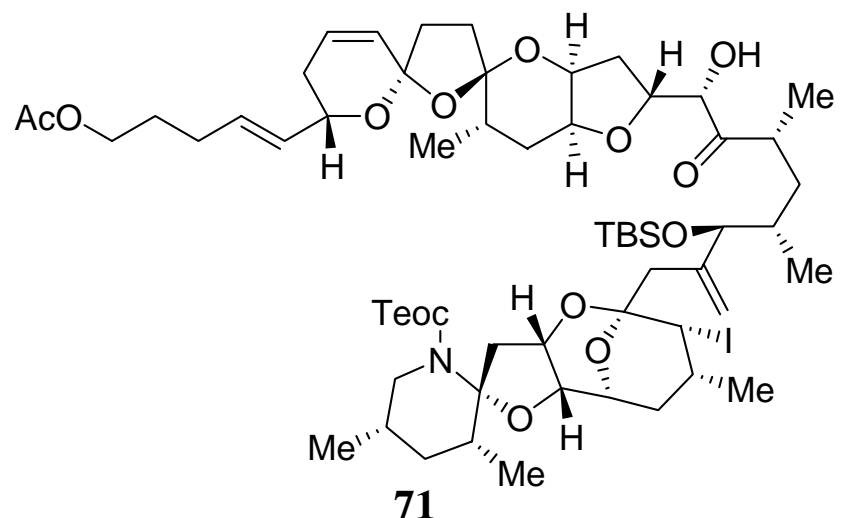
reaction mixture was stirred at $0{ }^{\circ} \mathrm{C}$ overnight and was then quenched with saturated aqueous $\mathrm{Na}_{2} \mathrm{~S}_{2} \mathrm{O}_{3}$ (10 $\mathrm{mL})$. The resulting biphasic mixture was extracted with $\mathrm{CH}_{2} \mathrm{Cl}_{2}(3 \times 10 \mathrm{~mL})$ and the combined organic extracts were dried $\left(\mathrm{Na}_{2} \mathrm{SO}_{4}\right)$, concentrated, and then purified by flash column chromatography (EtOAc:hexanes 1:2) to give iodoetherification product $71(12 \mathrm{mg}, 9.6 \mu \mathrm{mol}, 60 \%)$ as a colorless oil. 71: $R_{\mathrm{f}}=0.49$ (silica gel, EtOAc:hexanes 1:2); $[\alpha]_{\mathrm{D}}^{33}=-4.3\left(\mathrm{CH}_{3} \mathrm{OH}, c=0.4\right) ; \mathrm{IR}($ film $) v_{\max }=3446,2956,2927,1741,1699,1458,1396,1362,1251$, 
1176, $1071 \mathrm{~cm}^{-1} ;{ }^{1} \mathrm{H}$ NMR (500 MHz, $\left.\mathrm{CD}_{2} \mathrm{Cl}_{2}\right): \delta=6.00-5.91(\mathrm{~m}, 1 \mathrm{H}), 5.73-5.62(\mathrm{~m}, 2 \mathrm{H}), 5.50(\mathrm{dd}, J$ $=15.5,6.2 \mathrm{~Hz}, 1 \mathrm{H}), 5.41(\mathrm{~s}, 1 \mathrm{H}), 5.36(\mathrm{~s}, 1 \mathrm{H}), 4.86-4.77(\mathrm{~m}, 1 \mathrm{H}), 4.59(\mathrm{dd}, J=17.6,8.8 \mathrm{~Hz}, 1 \mathrm{H})$, 4.40 (d, J = 7.3 Hz, 1 H), 4.40-4.35 (m, $1 \mathrm{H}), 4.34$ (br s, $1 \mathrm{H}), 4.20-4.07$ (m, 5 H), 4.05 (br s, $1 \mathrm{H}), 4.01$ (t, $J=7.0 \mathrm{~Hz}, 2 \mathrm{H}), 3.99$ (br s, $1 \mathrm{H}), 3.82$ (br s, $1 \mathrm{H}), 3.71$ (d, $J=10.6 \mathrm{~Hz}, 1 \mathrm{H}), 3.18-3.07$ (m, $3 \mathrm{H})$, 3.07-2.99 (m, $1 \mathrm{H}), 2.65$ (d, $J=14.7 \mathrm{~Hz}, 1 \mathrm{H}), 2.50$ (dd, $J=14.7,8.8 \mathrm{~Hz}, 1 \mathrm{H}), 2.42-2.33$ (m, $1 \mathrm{H})$, 2.13-1.89 (m, 14 H), 1.90-1.80 (m, 2 H), 1.80-1.72 (m, 2 H), 1.72-1.65 (m, 2 H), 1.59-1.50 (m, $1 \mathrm{H})$, 1.48-1.41 (m, 1 H), 1.40-1.29 (m, 3 H), 1.26-1.19 (m, 1 H), 1.07 (d, J = 7.4 Hz, 3 H), 1.05-0.98 (m, 2 H), 0.96-0.90 (m, $16 \mathrm{H}), 0.84(\mathrm{~d}, J=7.0 \mathrm{~Hz}, 3 \mathrm{H}), 0.80$ (d, $J=6.6 \mathrm{~Hz}, 3 \mathrm{H}), 0.74(\mathrm{~d}, J=6.2 \mathrm{~Hz}, 3 \mathrm{H})$, $0.04(\mathrm{~s}, 12 \mathrm{H}),-0.02(\mathrm{~s}, 3 \mathrm{H}) ;{ }^{13} \mathrm{C}$ NMR $\left(125 \mathrm{MHz}, \mathrm{CD}_{2} \mathrm{Cl}_{2}\right): \delta=212.8,171.2,156.6,144.5,131.4$, 131.1, 129.7, 128.5, 117.1, 111.6, 104.3, 98.3, 97.3, 79.9, 79.6, 77.9, 77.2, 76.5, 76.2, 76.0, 73.3, 69.1, 64.0, 63.5, 50.4, 49.9, 45.7, 41.3, 39.0, 38.3, 37.7, 36.3, 36.2, 34.5, 33.2, 33.1, 32.1, 31.4, 31.4, 30.5, 29.0, 28.5, 26.9, 26.2, 24.0, 23.7, 21.1, 19.4, 18.7, 18.6, 18.1, 17.8, 16.6, 15.8, -1.4, -4.2, -4.7; HR-MS [electrospray ionization (ESI)]: calcd for $\mathrm{C}_{61} \mathrm{H}_{100} \mathrm{INO}_{14} \mathrm{Si}_{2} \mathrm{Na}^{+}\left[\mathrm{M}+\mathrm{Na}^{+}\right]:$1276.5619, found 1276.5630.

Reduction product 72. To a solution of iodoetherification product 9 (12 mg, $9.6 \mu \mathrm{mol}, 1.0$ equiv) in $n$ $\mathrm{Bu}_{3} \mathrm{SnH}$ :toluene $(1: 2,1.5 \mathrm{~mL})$ at $0{ }^{\circ} \mathrm{C}$ was added $\mathrm{Et}_{3} \mathrm{~B}(1.9 \mu \mathrm{L}, 1.0 \mathrm{M}$ in hexanes, $1.9 \mu \mathrm{mol}, 0.2$ equiv).

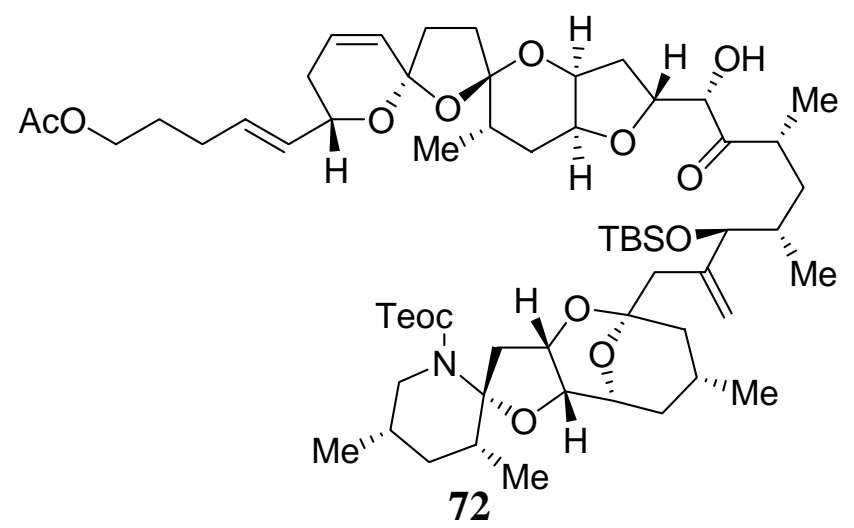

The reaction mixture was stirred at $0{ }^{\circ} \mathrm{C}$ for $20 \mathrm{~min}$, then directly purified by flash column chromatography (silica gel, EtOAc:hexanes 1:2) to provide reduction product $72(6 \mathrm{mg}, 5.3 \mu \mathrm{mol}, 55 \%)$ as a colorless oil. 72: $R_{\mathrm{f}}=0.44$ (silica gel, EtOAc:hexanes 1:2); $[\alpha]_{\mathrm{D}}^{35}=-12.3\left(\mathrm{CH}_{3} \mathrm{OH}, c=\right.$ 0.3); IR (film) $v_{\max }=3460,2955,2921,2845,1739$,

1694, 1463, 1393, 1251, 1172, $1072 \mathrm{~cm}^{-1} ;{ }^{1} \mathrm{H}$ NMR (500 MHz, $\left.\mathrm{CD}_{2} \mathrm{Cl}_{2}\right): \delta=6.00-5.92(\mathrm{~m}, 1 \mathrm{H}), 5.72-$ $5.62(\mathrm{~m}, 1 \mathrm{H}), 5.69-5.60(\mathrm{~m}, 1 \mathrm{H}), 5.49(\mathrm{dd}, J=15.8,6.3 \mathrm{~Hz}, 1 \mathrm{H}), 5.16(\mathrm{~s}, 1 \mathrm{H}), 5.14(\mathrm{~s}, 1 \mathrm{H}), 4.78-$ 4.70 (m, $1 \mathrm{H}), 4.69-4.60$ (m, $1 \mathrm{H}), 4.40-4.31(\mathrm{~m}, 1 \mathrm{H}), 4.22$ (d, J = 4.1 Hz, $1 \mathrm{H}), 4.19-4.11$ (m, $3 \mathrm{H})$, 4.07-4.02 (m, $1 \mathrm{H}), 4.01$ (t, $J=6.6 \mathrm{~Hz}, 2 \mathrm{H}), 3.96$ (br s, $1 \mathrm{H}), 3.90$ (d, $J=6.3 \mathrm{~Hz}, 1 \mathrm{H}), 3.82$ (br s, $1 \mathrm{H})$, 
$3.79(\mathrm{~d}, J=5.9 \mathrm{~Hz}, 1 \mathrm{H}), 3.72(\mathrm{~d}, J=13.6 \mathrm{~Hz}, 1 \mathrm{H}), 3.43(\mathrm{dd}, J=14.4,4.1 \mathrm{~Hz}, 1 \mathrm{H}), 3.19(\mathrm{dd}, J=13.2$, $11.8 \mathrm{~Hz}, 1 \mathrm{H}), 2.99-2.92(\mathrm{~m}, 1 \mathrm{H}), 2.40-2.32(\mathrm{~m}, 1 \mathrm{H}), 2.31(\mathrm{dd}, J=14.7,6.6 \mathrm{~Hz}, 1 \mathrm{H}), 2.26(\mathrm{~d}, J=14.7$ Hz, $1 \mathrm{H}), 2.17-1.86$ (m, $18 \mathrm{H}), 1.84-1.74(\mathrm{~m}, 1 \mathrm{H}), 1.72-1.58$ (m, $4 \mathrm{H}), 1.56-1.47$ (m, $2 \mathrm{H}), 1.44-1.31$ (m, $2 \mathrm{H}), 1.30-1.20(\mathrm{~m}, 2 \mathrm{H}), 1.06(\mathrm{~d}, J=7.0 \mathrm{~Hz}, 3 \mathrm{H}), 1.02-0.94(\mathrm{~m}, 2 \mathrm{H}), 0.93(\mathrm{~s}, 9 \mathrm{H}), 0.92-0.86(\mathrm{~m}$, $7 \mathrm{H}), 0.84(\mathrm{~d}, J=6.6 \mathrm{~Hz}, 3 \mathrm{H}), 0.81(\mathrm{~d}, J=6.6 \mathrm{~Hz}, 3 \mathrm{H}), 0.74(\mathrm{~d}, J=6.2 \mathrm{~Hz}, 3 \mathrm{H}), 0.04(\mathrm{~s}, 3 \mathrm{H}), 0.03$ (s, $9 \mathrm{H}),-0.01(\mathrm{~s}, 3 \mathrm{H}) ;{ }^{13} \mathrm{C} \mathrm{NMR}\left(125 \mathrm{MHz}, \mathrm{CD}_{2} \mathrm{Cl}_{2}\right): \delta=213.2,171.1,156.6,145.4,131.4,131.1,129.6$, $128.5,114.7,111.7,104.3,97.2,80.0,78.9,78.4,77.5,76.5,76.3,73.7,72.8,69.0,64.0,63.1,49.4$, $45.7,42.0,41.1,39.4,39.1,37.2,36.4,36.2,35.9,32.2,33.6,32.9,31.6,31.4,30.5,29.0,28.5,26.2$, 24.4, 23.7, 23.0, 21.1, 19.5, 18.8, 18.5, 18.2, 18.0, 16.7, 15.8, -1.4, -4.0, -4.8; HR-MS [electrospray ionization (ESI)]: calcd for $\mathrm{C}_{61} \mathrm{H}_{101} \mathrm{NO}_{14} \mathrm{Si}_{2} \mathrm{Na}^{+}\left[\mathrm{M}+\mathrm{Na}^{+}\right]$: 1150.6652 , found 1150.6640 .

TES protected product 73. To a solution of reduction product 72 (7 mg, $6.2 \mu \mathrm{mol}, 1.0$ equiv) in $\mathrm{CH}_{2} \mathrm{Cl}_{2}(1 \mathrm{~mL})$ at $-78{ }^{\circ} \mathrm{C}$ was added 2,6-lutidine $(14 \mu \mathrm{L}, 0.124 \mathrm{mmol}, 20$ equiv) followed by the

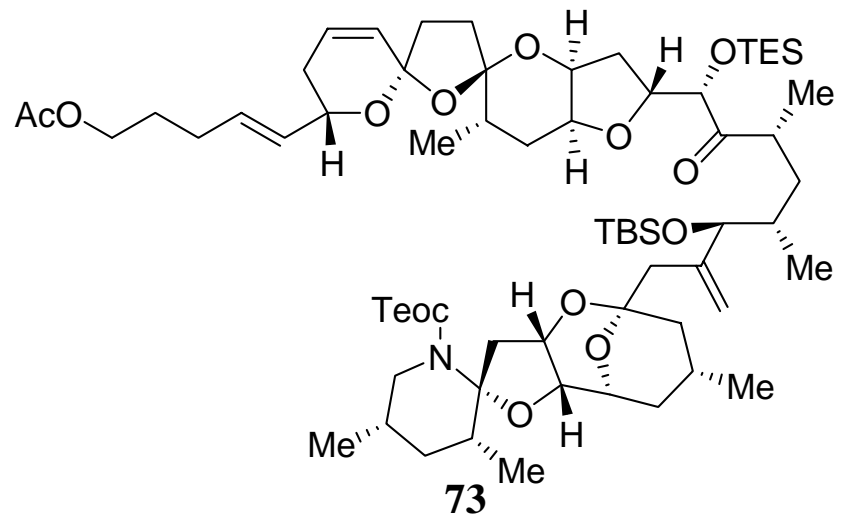
dropwise addition of TESOTf $(14 \mu \mathrm{L}, 62 \mu \mathrm{mol}, 10$ equiv). The reaction mixture was warmed to $0{ }^{\circ} \mathrm{C}$ over $30 \mathrm{~min}$, then quenched with saturated aqueous $\mathrm{NH}_{4} \mathrm{Cl}(5 \mathrm{ml})$. The resulting biphasic mixture was extracted with $\mathrm{CH}_{2} \mathrm{Cl}_{2}(3 \times 5 \mathrm{~mL})$ and the combined organic extracts were dried $\left(\mathrm{Na}_{2} \mathrm{SO}_{4}\right)$, concentrated, and then purified by flash column chromatography (EtOAc:hexanes 1:3) to give TES protected product $73(7 \mathrm{mg}, 5.6 \mu \mathrm{mol}, 90 \%)$ as a colorless oil. 73: $R_{\mathrm{f}}$ $=0.36$ (silica gel, EtOAc:hexanes 1:4); $[\alpha]_{\mathrm{D}}^{34}=-13.5\left(\mathrm{CH}_{3} \mathrm{OH}, c=0.4\right) ; \mathrm{IR}\left(\right.$ film) $v_{\max }=3447,2955$, 2926, 2875, 1740, 1700, 1458, 1397, 1250, 1171, 1133, $1062 \mathrm{~cm}^{-1} ;{ }^{1} \mathrm{H}$ NMR $\left(600 \mathrm{MHz}, \mathrm{CD}_{2} \mathrm{Cl}_{2}\right): \delta=$ 6.00-5.91 (m, $1 \mathrm{H}), 5.71-5.61(\mathrm{~m}, 2 \mathrm{H}), 5.50(\mathrm{dd}, J=15.4,6.1 \mathrm{~Hz}, 1 \mathrm{H}), 5.13$ (br s, $2 \mathrm{H}), 4.73-4.64$ (m, $1 \mathrm{H}), 4.61-4.53(\mathrm{~m}, 1 \mathrm{H}), 4.39-4.31(\mathrm{~m}, 1 \mathrm{H}), 4.23(\mathrm{~d}, J=4.0 \mathrm{~Hz}, 1 \mathrm{H}), 4.22-4.17(\mathrm{~m}, 1 \mathrm{H}), 4.15(\mathrm{~d}, J=$ $4.4 \mathrm{~Hz}, 1 \mathrm{H}), 4.11$ (br s, $1 \mathrm{H}), 4.07-3.97$ (m, 3 H), 3.95 (br s, $1 \mathrm{H}), 3.76$ (br s, $1 \mathrm{H}), 3.73$ (d, J=4.9 Hz, 1 H), 3.73-3.67 (m, $1 \mathrm{H}), 3.51$ (d, $J=14.0 \mathrm{~Hz}, 1 \mathrm{H}), 3.19$ (t, $J=12.7 \mathrm{~Hz}, 1 \mathrm{H}), 2.99-2.88(\mathrm{~m}, 1 \mathrm{H}), 2.29$ 
$(\mathrm{dd}, J=14.5,5.7 \mathrm{~Hz}, 1 \mathrm{H}), 2.21(\mathrm{~d}, J=14.5 \mathrm{~Hz}, 1 \mathrm{H}), 2.13-1.85(\mathrm{~m}, 21 \mathrm{H}), 1.72-1.62(\mathrm{~m}, 3 \mathrm{H}), 1.60-$ $1.48(\mathrm{~m}, 3 \mathrm{H}), 1.49-1.42(\mathrm{~m}, 1 \mathrm{H}), 1.41-1.35(\mathrm{~m}, 1 \mathrm{H}), 1.33-1.27$ (m, $1 \mathrm{H}), 1.26-1.22(\mathrm{~m}, 1 \mathrm{H}), 1.03$ (d, $J=7.0 \mathrm{~Hz}, 3 \mathrm{H}), 0.98(\mathrm{~m}, 2 \mathrm{H}), 0.95$ (t, $J=8.3 \mathrm{~Hz}, 9 \mathrm{H}), 0.92(\mathrm{~s}, 9 \mathrm{H}), 0.89$ (d, J = 6.6 Hz, $3 \mathrm{H}), 0.86$ (d, $J=7.0 \mathrm{~Hz}, 3 \mathrm{H}), 0.85(\mathrm{~d}, J=7.0 \mathrm{~Hz}, 3 \mathrm{H}), 0.86-0.82(\mathrm{~m}, 1 \mathrm{H}), 0.81(\mathrm{~d}, J=6.5 \mathrm{~Hz}, 3 \mathrm{H}), 0.80(\mathrm{~d}, J=6.1$ $\mathrm{Hz}, 3 \mathrm{H}), 0.62$ (q, $J=7.9 \mathrm{~Hz}, 6 \mathrm{H}), 0.04(\mathrm{~s}, 12 \mathrm{H}),-0.01(\mathrm{~s}, 3 \mathrm{H}) ;{ }^{13} \mathrm{C} \mathrm{NMR}\left(125 \mathrm{MHz}, \mathrm{CD}_{2} \mathrm{Cl}_{2}\right)$ : $\delta=212.9,171.2,156.5,145.1,131.4,131.1,129.7,128.5,114.9,111.8,104.3,97.2,96.9,80.2,79.9$, $79.7,78.5,76.4,76.3,73.8,71.9,69.0,64.0,62.9,49.3,45.2,42.0,40.8,40.1,39.3,37.1,36.4,36.1$, $35.7,34.3,34.1,33.3,31.6,31.3,30.5,29.0,28.5,26.2,25.0,23.6,23.2,21.1,18.8,18.7,18.5,18.5$, 18.0, 16.8, 15.8, 7.1, 5.3, -1.4, -4.0, -4.8; HR-MS [electrospray ionization (ESI)]: calcd for $\mathrm{C}_{67} \mathrm{H}_{115} \mathrm{NO}_{14} \mathrm{Si}_{3} \mathrm{Na}^{+}\left[\mathrm{M}+\mathrm{Na}^{+}\right]:$1264.7517, found 1264.7510 .

Alcohol 74. To a solution of TES protected product $73(10 \mathrm{mg}, 8.0 \mu \mathrm{mol}, 1.0$ equiv) in $\mathrm{MeOH}(1 \mathrm{~mL})$ at $25^{\circ} \mathrm{C}$ was added solid $\mathrm{K}_{2} \mathrm{CO}_{3}\left(4 \mathrm{mg}, 29 \mu \mathrm{mol}, 3.6\right.$ equiv). The mixture was stirred at $25^{\circ} \mathrm{C}$ for $2 \mathrm{~h}$, then

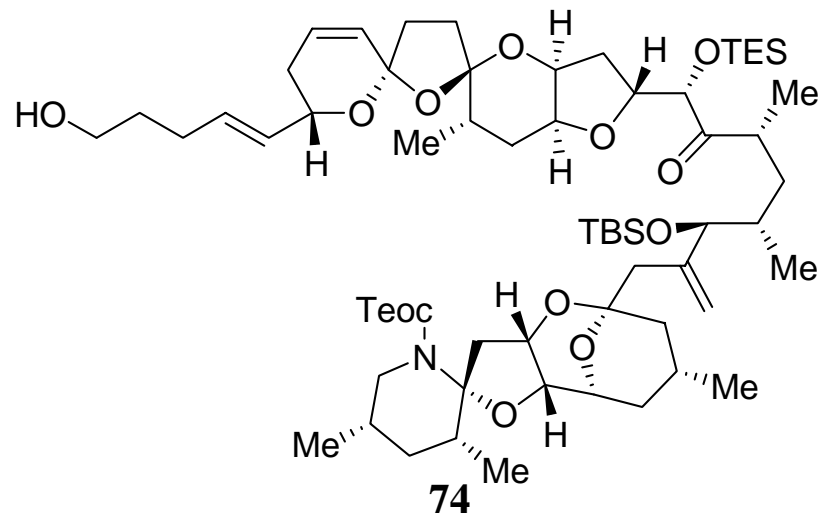
the reaction was quenched with saturated aqueous $\mathrm{NH}_{4} \mathrm{Cl}(5 \mathrm{~mL})$. The resulting biphasic mixture was extracted with $\mathrm{CH}_{2} \mathrm{Cl}_{2}(3 \times 5 \mathrm{~mL})$ and the combined organic extracts were dried $\left(\mathrm{Na}_{2} \mathrm{SO}_{4}\right)$, concentrated, and then purified by flash column chromatography (EtOAc:hexanes 1:1) to give alcohol $74(7.5 \mathrm{mg}, 6.3$ $\mu \mathrm{mol}, 79 \%$ ) as a colorless oil. 74: $R_{\mathrm{f}}=0.25$ (silica gel, EtOAc:hexanes 1:2); $[\alpha]_{\mathrm{D}}{ }^{33}=-14.7\left(\mathrm{CH}_{3} \mathrm{OH}, c=0.15\right)$; IR (film) $v_{\max }=3510,2955,2928,2870,2850$, 1718, 1676, 1458, 1397, 1252, $1171 \mathrm{~cm}^{-1} ;{ }^{1} \mathrm{H}$ NMR (500 MHz, $\left.\mathrm{CD}_{2} \mathrm{Cl}_{2}\right): \delta=6.00-5.92(\mathrm{~m}, 1 \mathrm{H}), 5.73-$ $5.60(\mathrm{~m}, 2 \mathrm{H}), 5.49(\mathrm{dd}, J=15.8,6.2 \mathrm{~Hz}, 1 \mathrm{H}), 5.13(\mathrm{~s}, 2 \mathrm{H}), 4.72-4.64(\mathrm{~m}, 1 \mathrm{H}), 4.62-4.52(\mathrm{~m}, 1 \mathrm{H})$, 4.40-4.31 (m, 1 H), 4.23 (d, J=4.4 Hz, $1 \mathrm{H}), 4.20-4.15$ (m, $1 \mathrm{H}), 4.16$ (d, J=4.4 Hz, $1 \mathrm{H}), 4.12$ (br s, 1 H), 4.04-3.97 (m, $1 \mathrm{H}), 3.95$ (d, $J=3.1 \mathrm{~Hz}, 1 \mathrm{H}), 3.77$ (br s, $1 \mathrm{H}), 3.74(\mathrm{~d}, J=4.8 \mathrm{~Hz}, 1 \mathrm{H}), 3.72-3.66$ (m, $1 \mathrm{H}), 3.59$ (t, $J=6.6 \mathrm{~Hz}, 2 \mathrm{H}), 3.51$ (d, $J=14.7 \mathrm{~Hz}, 1 \mathrm{H}), 3.19$ (t, $J=12.9 \mathrm{~Hz}, 1 \mathrm{H}), 2.99-2.91(\mathrm{~m}, 1$ H), $2.89(\mathrm{dd}, J=14.7,5.9 \mathrm{~Hz}, 1 \mathrm{H}), 2.22(\mathrm{~d}, J=14.7 \mathrm{~Hz}, 1 \mathrm{H}), 2.14-1.84(\mathrm{~m}, 17 \mathrm{H}), 1.71-1.51$ (m, 6 
H), 1.47-1.34 (m, $2 \mathrm{H}), 1.36-1.19(\mathrm{~m}, 2 \mathrm{H}), 1.03(\mathrm{~d}, J=7.0 \mathrm{~Hz}, 3 \mathrm{H}), 0.99(\mathrm{~m}, 2 \mathrm{H}), 0.95(\mathrm{t}, J=8.1 \mathrm{~Hz}$, $9 \mathrm{H}), 0.92(\mathrm{~s}, 9 \mathrm{H}), 0.89$ (d, J=6.3 Hz, $3 \mathrm{H}), 0.86$ (d, J=6.6 Hz, $3 \mathrm{H}), 0.85$ (d, J=6.6 Hz, $3 \mathrm{H}), 0.85-$ $0.83(\mathrm{~m}, 1 \mathrm{H}), 0.81(\mathrm{~d}, J=6.6 \mathrm{~Hz}, 3 \mathrm{H}), 0.80(\mathrm{~d}, J=6.3 \mathrm{~Hz}, 3 \mathrm{H}), 0.62(\mathrm{q}, J=8.1 \mathrm{~Hz}, 6 \mathrm{H}), 0.04(\mathrm{~s}, 12$ $\mathrm{H}),-0.01(\mathrm{~s}, 3 \mathrm{H}) ;{ }^{13} \mathrm{C} \mathrm{NMR}\left(125 \mathrm{MHz}, \mathrm{CD}_{2} \mathrm{Cl}_{2}\right): \delta=212.8,156.5,145.1,131.9,131.0,129.7,128.5$, $114.9,111.8,104.3,97.2,96.9,80.2,79.9,79.7,78.5,76.5,76.4,73.8,71.9,69.1,62.9,62.4,49.3,45.2$, $42.0,40.8,40.1,39.3,37.1,36.4,36.1,35.7,34.3,34.1,33.3,32.5,31.6,31.3,30.5,29.0,26.2,25.0$, 23.6, 23.2, 18.8, 18.7, 18.5, 18.5, 18.0, 16.8, 15.8, 7.1, 5.3,-1.4, -4.0, -4.8; HR-MS [electrospray ionization (ESI)]: calcd for $\mathrm{C}_{65} \mathrm{H}_{113} \mathrm{NO}_{13} \mathrm{Si}_{3} \mathrm{H}^{+}\left[\mathrm{M}+\mathrm{H}^{+}\right]$: 1200.7592, found 1200.7601 .

Carboxylic acid 75. To a solution of DMSO $(39 \mu \mathrm{L}, 0.55 \mathrm{mmol}, 110$ equiv $)$ in $\mathrm{CH}_{2} \mathrm{Cl}_{2}(1 \mathrm{~mL})$ at $-78{ }^{\circ} \mathrm{C}$ was added oxalyl chloride $\left(22 \mu \mathrm{L}, 0.25 \mathrm{mmol}, 50\right.$ equiv). The mixture was kept at $-78{ }^{\circ} \mathrm{C}$ for $15 \mathrm{~min}$

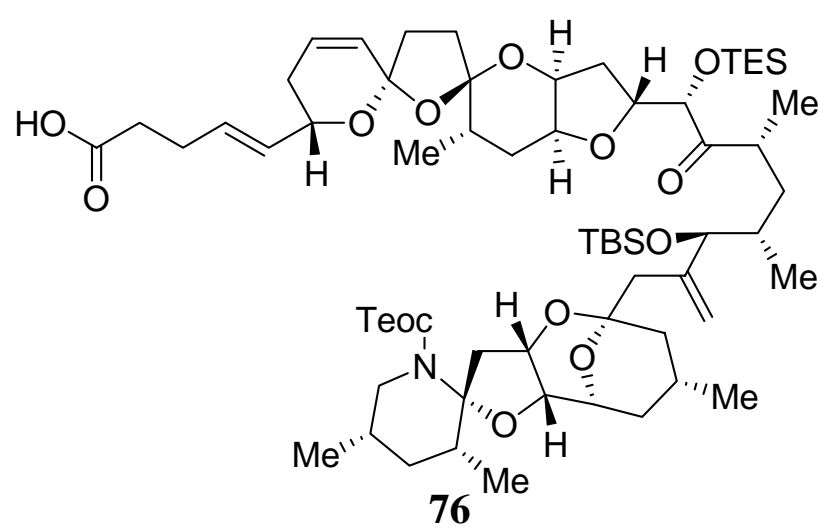
and then alcohol 74 (6 $\mathrm{mg}, 5 \mu \mathrm{mol}, 1.0$ equiv) in $\mathrm{CH}_{2} \mathrm{Cl}_{2}(1.5 \mathrm{~mL})$ was added. The reaction mixture was maintained at $-78{ }^{\circ} \mathrm{C}$ for $1 \mathrm{~h}$ then $\mathrm{Et}_{3} \mathrm{~N}(153 \mu \mathrm{L}, 1.1$ mmol, 220 equiv) was added and the solution was warmed to $0{ }^{\circ} \mathrm{C}$ over $1 \mathrm{~h}$. The reaction mixture was then quenched with saturated aqueous $\mathrm{NaCl}(5 \mathrm{~mL})$, extracted with $\mathrm{CH}_{2} \mathrm{Cl}_{2}(3 \times 5 \mathrm{~mL})$ and the combined

organic extracts were dried $\left(\mathrm{Na}_{2} \mathrm{SO}_{4}\right)$ and concentrated. The crude product was dissolved in $t$ $\mathrm{BuOH}: \mathrm{H}_{2} \mathrm{O}(4: 1,1 \mathrm{~mL})$ at $25{ }^{\circ} \mathrm{C}$ and to the solution was added $\mathrm{NaH}_{2} \mathrm{PO}_{4}(15 \mathrm{mg}), 2$-methyl-2-butene $(100 \mu \mathrm{L})$, and $\mathrm{NaClO}_{2}(7.5 \mathrm{mg})$. The reaction mixture was maintained at $25{ }^{\circ} \mathrm{C}$ for $1 \mathrm{~h}$, and was then quenched with a pH 7 buffered saline solution $(10 \mathrm{~mL})$. The resulting biphasic mixture was extracted with $\mathrm{CH}_{2} \mathrm{Cl}_{2}(3 \times 5 \mathrm{~mL})$, dried $\left(\mathrm{Na}_{2} \mathrm{SO}_{4}\right)$, and concentrated to give carboxylic acid 75 (4.5 mg, $4 \mu \mathrm{mol}$, $80 \%$ over two steps) as a colorless oil. 75: $R_{\mathrm{f}}=0.43$ (silica gel, $\mathrm{CH}_{2} \mathrm{Cl}_{2}: \mathrm{MeOH} 10: 1$ ); $[\alpha]_{\mathrm{D}}{ }^{33}=-12.5$ $\left(\mathrm{CH}_{3} \mathrm{OH}, c=0.4\right)$; IR (film) $v_{\max }=2944,2926,2876,1700,1458,1250,1170,1059 \mathrm{~cm}^{-1} ;{ }^{1} \mathrm{H}$ NMR $\left(600 \mathrm{MHz}, \mathrm{CD}_{2} \mathrm{Cl}_{2}\right): \delta=6.00-5.95(\mathrm{~m}, 1 \mathrm{H}), 5.74-5.67(\mathrm{~m}, 1 \mathrm{H}), 5.63(\mathrm{~d}, J=10.1 \mathrm{~Hz}, 1 \mathrm{H}), 5.53(\mathrm{dd}, J$ $=15.8,6.1 \mathrm{~Hz}, 1 \mathrm{H}), 5.13(\mathrm{~s}, 3 \mathrm{H}), 4.73-4.67(\mathrm{~m}, 1 \mathrm{H}), 4.62-4.53(\mathrm{~m}, 1 \mathrm{H}), 4.40-4.34(\mathrm{~m}, 1 \mathrm{H}), 4.24(\mathrm{~d}$, 
$J=4.0 \mathrm{~Hz}, 1 \mathrm{H}), 4.17(\mathrm{~d}, J=4.4 \mathrm{~Hz}, 1 \mathrm{H}), 4.20-4.12(\mathrm{~m}, 1 \mathrm{H}), 4.12(\mathrm{br} \mathrm{s}, 1 \mathrm{H}), 4.04-3.96(\mathrm{~m}, 1 \mathrm{H}), 3.94$ (d, $J=3.5 \mathrm{~Hz}, 1 \mathrm{H}), 3.77(\mathrm{br} \mathrm{s}, 1 \mathrm{H}), 3.74(\mathrm{~d}, J=4.9 \mathrm{~Hz}, 1 \mathrm{H}), 3.72(\mathrm{~m}, 1 \mathrm{H}), 3.50(\mathrm{~d}, J=14.8 \mathrm{~Hz}, 1 \mathrm{H})$, $3.19(\mathrm{t}, J=13.2 \mathrm{~Hz}, 1 \mathrm{H}), 2.98-2.90(\mathrm{~m}, 1 \mathrm{H}), 2.41(\mathrm{t}, J=7.6 \mathrm{~Hz}, 2 \mathrm{H}), 2.35(\mathrm{t}, J=7.1 \mathrm{~Hz}, 2 \mathrm{H}), 2.30$ (dd, $J=15.0,6.2 \mathrm{~Hz}, 1 \mathrm{H}), 2.21$ (d, $J=14.5 \mathrm{~Hz}, 1 \mathrm{H}), 2.15-1.86$ (m, $15 \mathrm{H}), 1.71-1.66$ (m, $1 \mathrm{H}), 1.64-$ 1.47 (m, 3 H), 1.43 (m, 1 H), 1.38-1.20 (m, 3 H), 1.03 (d, J = 7.0 Hz, 3 H), 1.02-0.96 (m, 2 H), 0.94 (t, $J=7.9 \mathrm{~Hz}, 9 \mathrm{H}), 0.92(\mathrm{~s}, 9 \mathrm{H}), 0.89$ (d, $J=6.1 \mathrm{~Hz}, 3 \mathrm{H}), 0.86(\mathrm{~d}, J=7.0 \mathrm{~Hz}, 3 \mathrm{H}), 0.84(\mathrm{~d}, J=6.6 \mathrm{~Hz}, 3$ H), $0.88-0.80(\mathrm{~m}, 1 \mathrm{H}), 0.82(\mathrm{~d}, J=6.6 \mathrm{~Hz}, 3 \mathrm{H}), 0.81(\mathrm{~d}, J=6.2 \mathrm{~Hz}, 3 \mathrm{H}), 0.62(\mathrm{q}, J=7.9 \mathrm{~Hz}, 6 \mathrm{H})$, 0.04 (s, $3 \mathrm{H}), 0.03(\mathrm{~s}, 9 \mathrm{H}),-0.01(\mathrm{~s}, 3 \mathrm{H}) ;{ }^{13} \mathrm{C}$ NMR $\left(125 \mathrm{MHz}, \mathrm{CD}_{2} \mathrm{Cl}_{2}\right): \delta=212.7,156.5,145.2$, 131.8, 130.1, 129.7, 128.5, 115.0, 111.8, 104.3, 97.3, 97.2, 80.3, 79.7, 78.5, 76.4, 76.4, 73.8, 71.9, 69.0, 63.1, 49.4, 45.3, 42.1, 40.8, 40.1, 39.3, 37.3, 34.6, 31.6, 31.4, 36.4, 36.2, 35.7, 34.6, 33.3. 30.5, 30.1, $30.1,23.7,26.3,25.0,23.6,23.3,18.9,18.8,18.5,18.6,18.1,16.8,15.8,7.1,5.4,-1.4,-4.0,-4.8$; HRMS [electrospray ionization (ESI)]: calcd for $\mathrm{C}_{65} \mathrm{H}_{111} \mathrm{NO}_{14} \mathrm{Si}_{3} \mathrm{Na}^{+}\left[\mathrm{M}+\mathrm{Na}^{+}\right]$: 1236.7204 , found 1236.7203 .

References:

(1) Satake, M.; Ofuji, K.; Naoki, H.; James, K. J.; Furey, A.; McMahon, T.; Silke, J.; Yasumoto, T. J. Am. Chem. Soc. 1998, 120, 9967. 


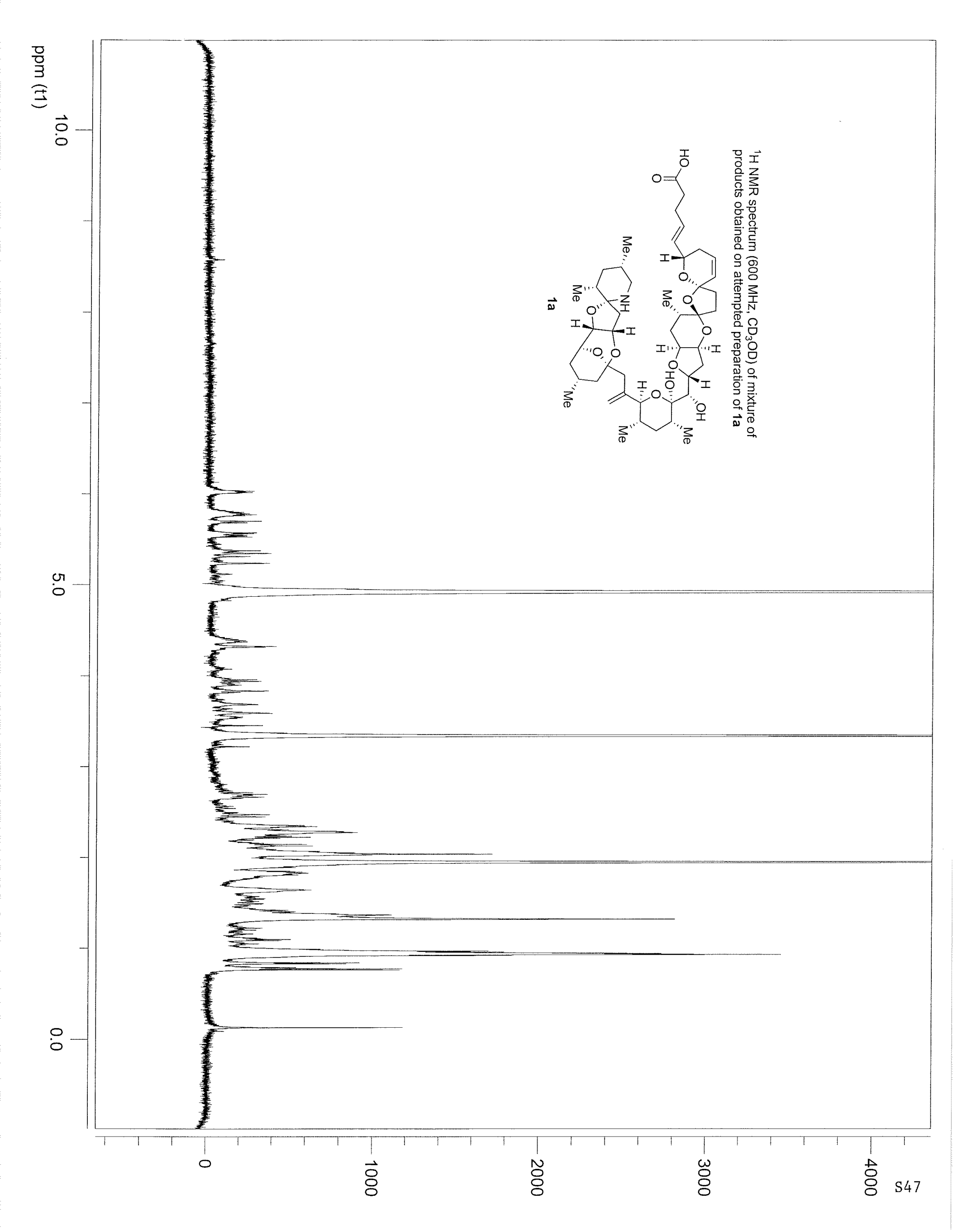



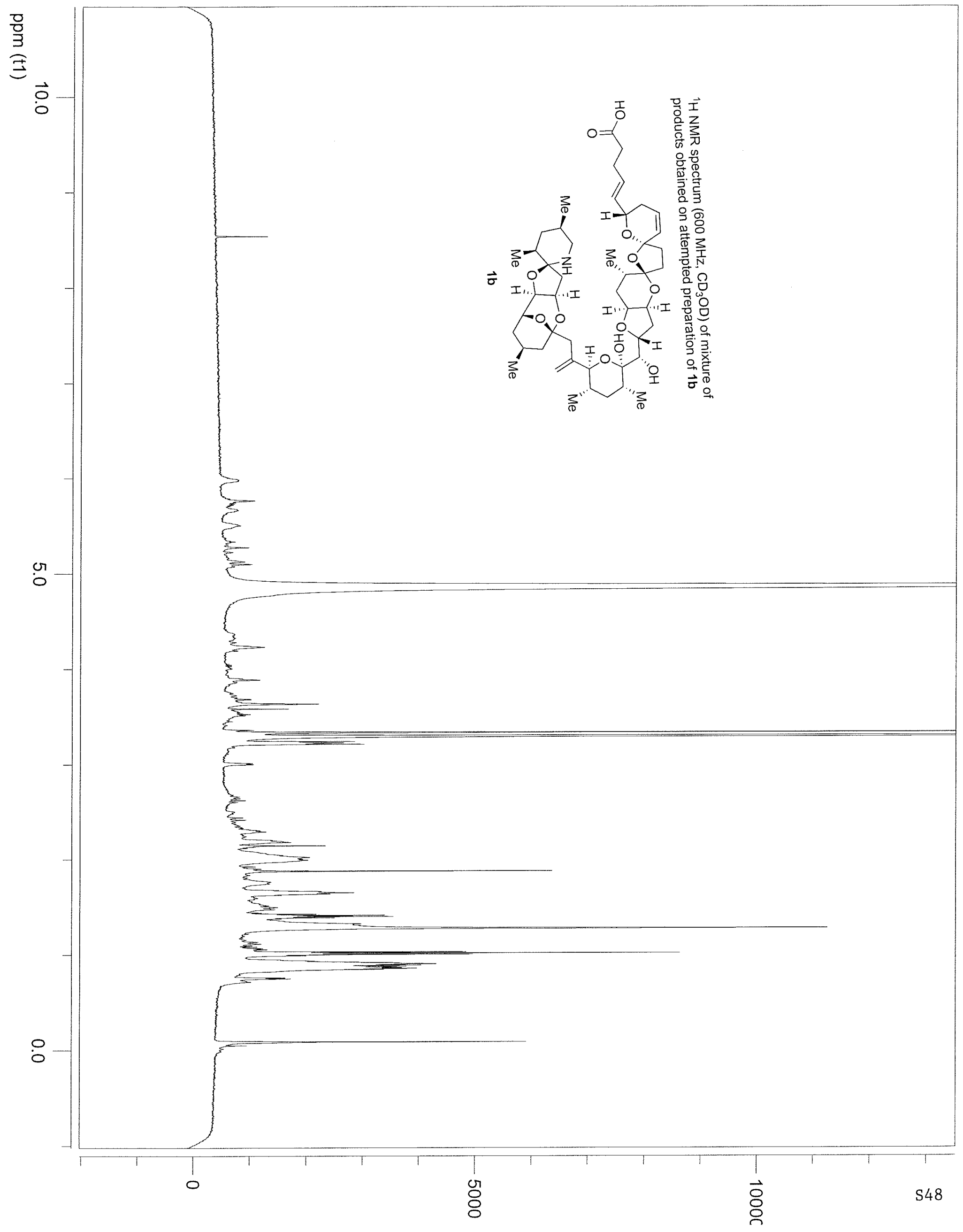


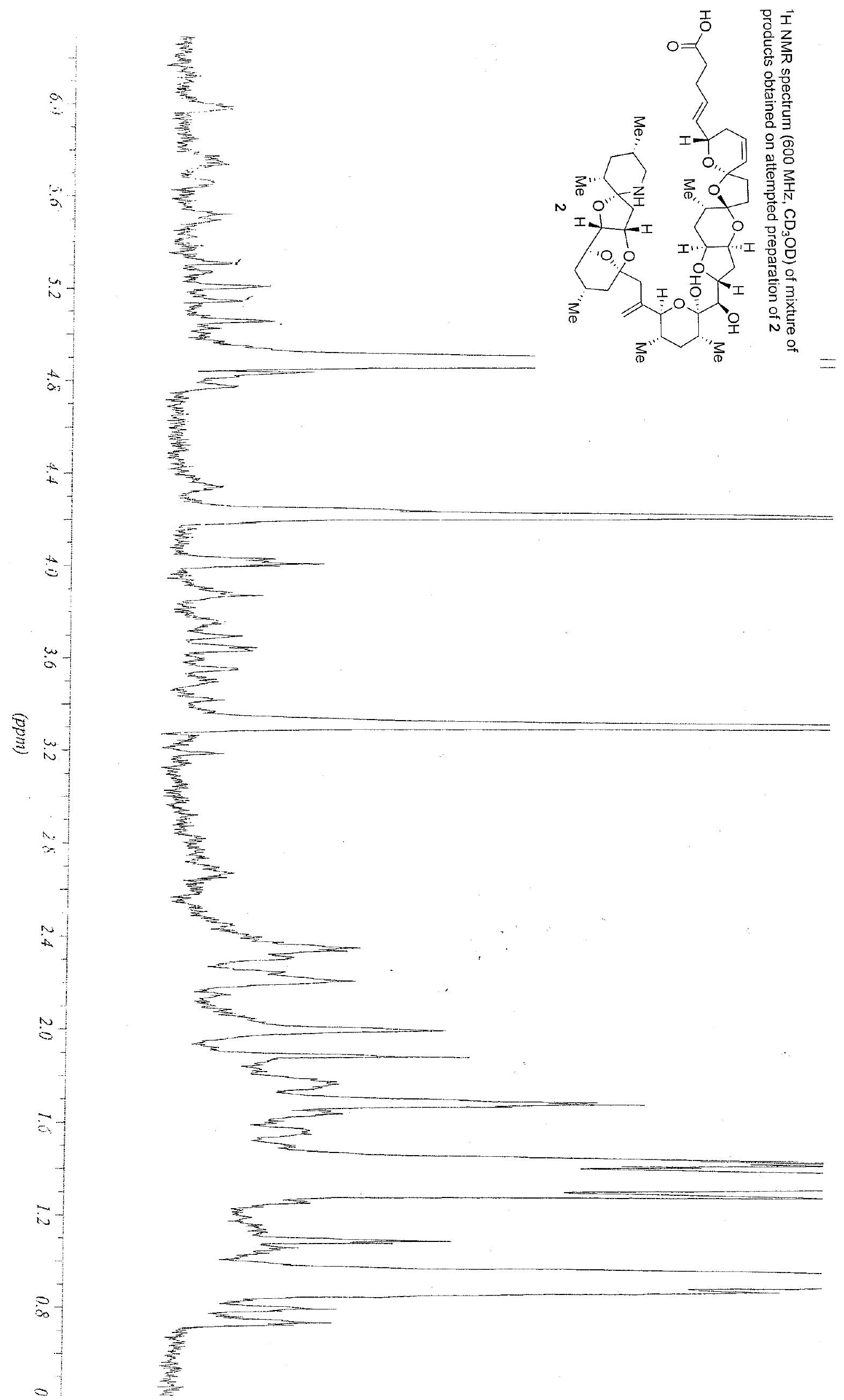




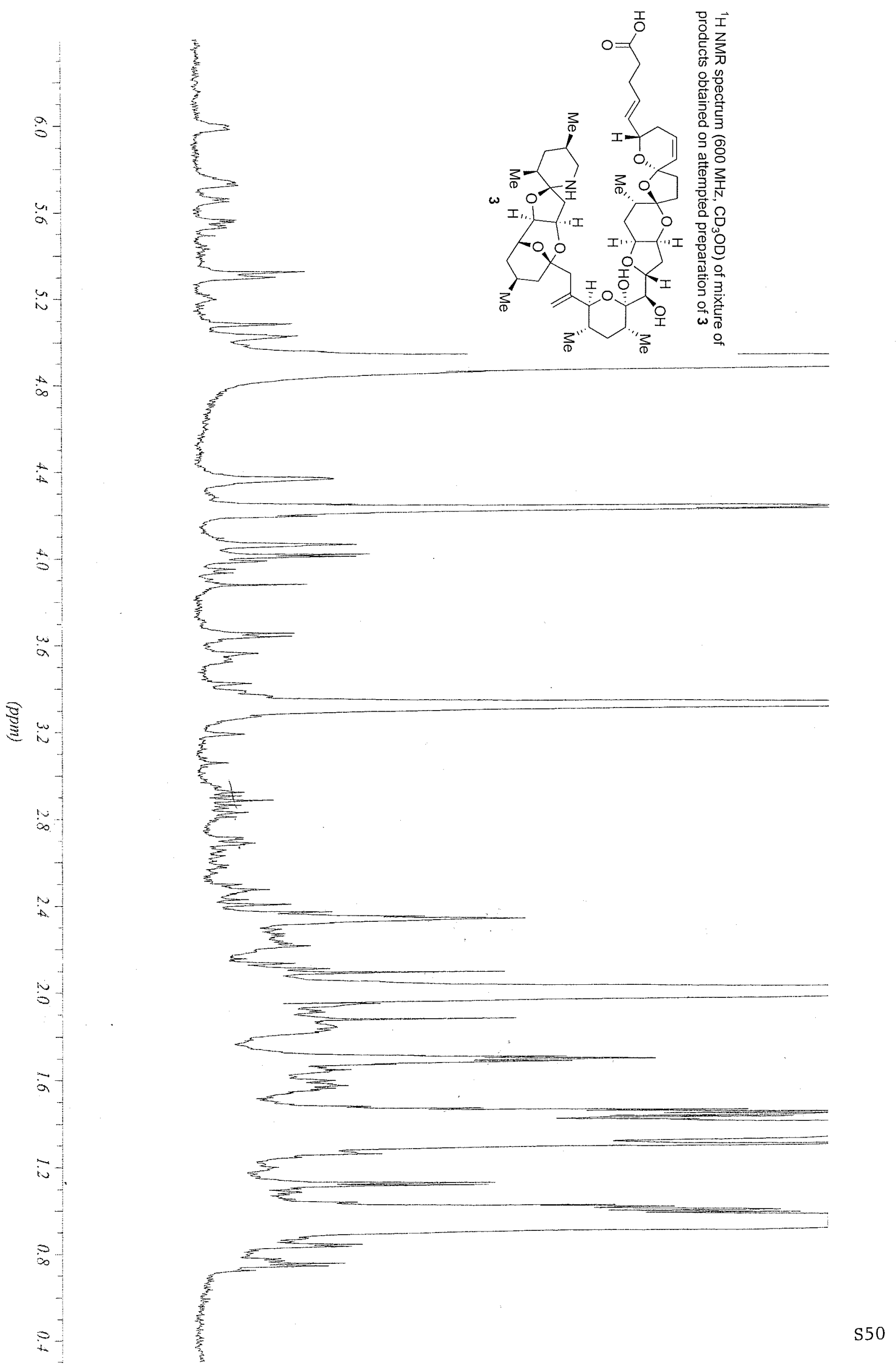




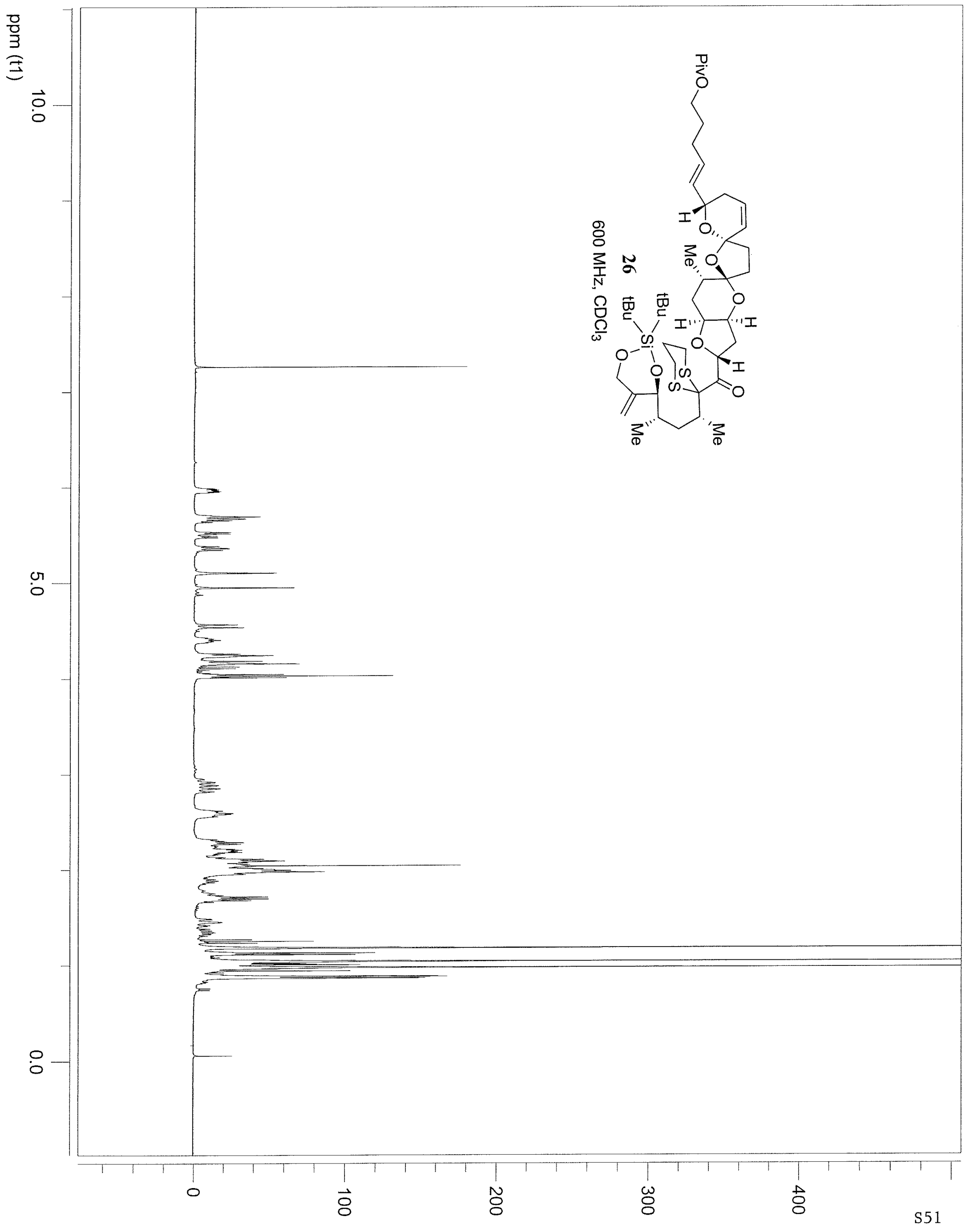




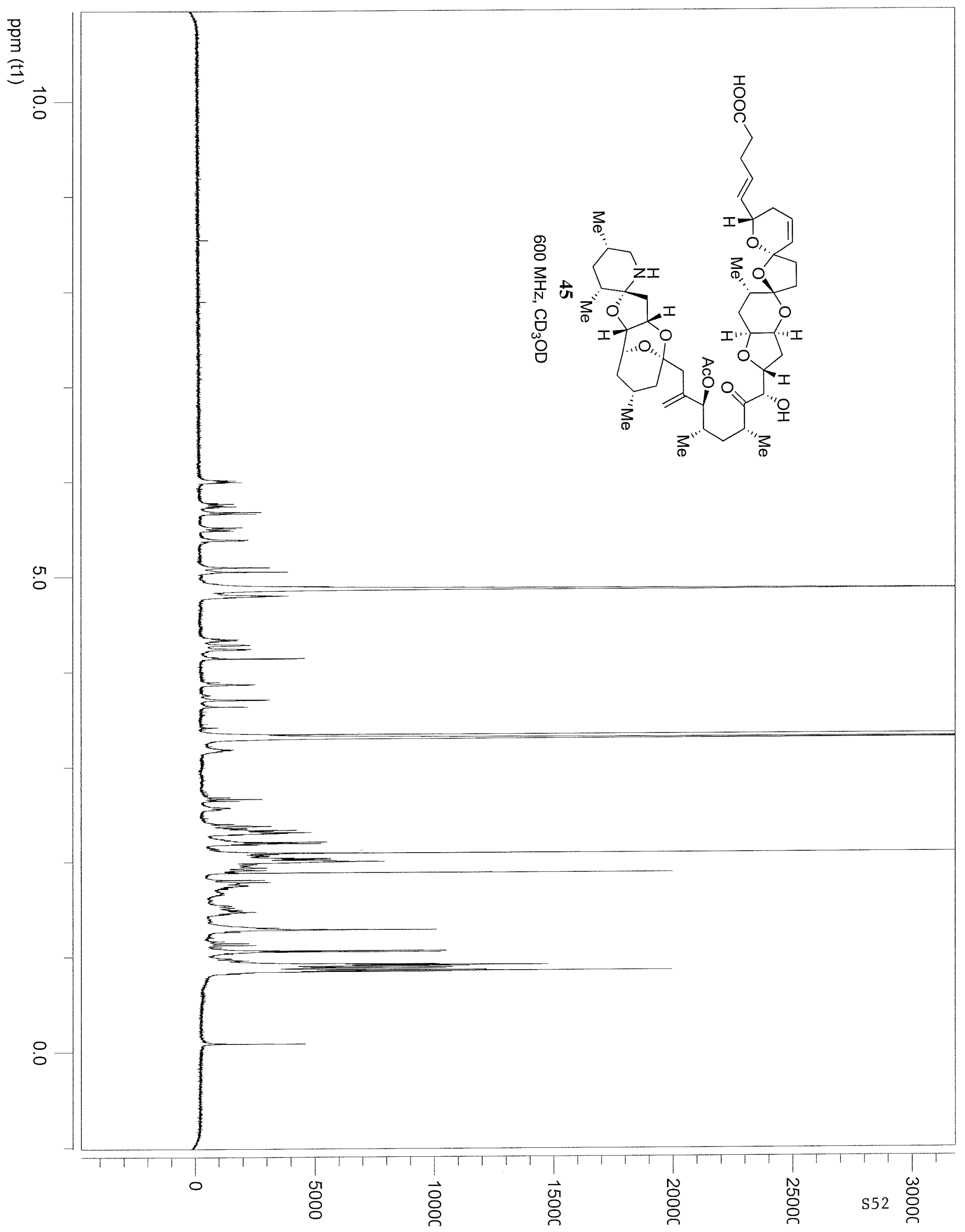




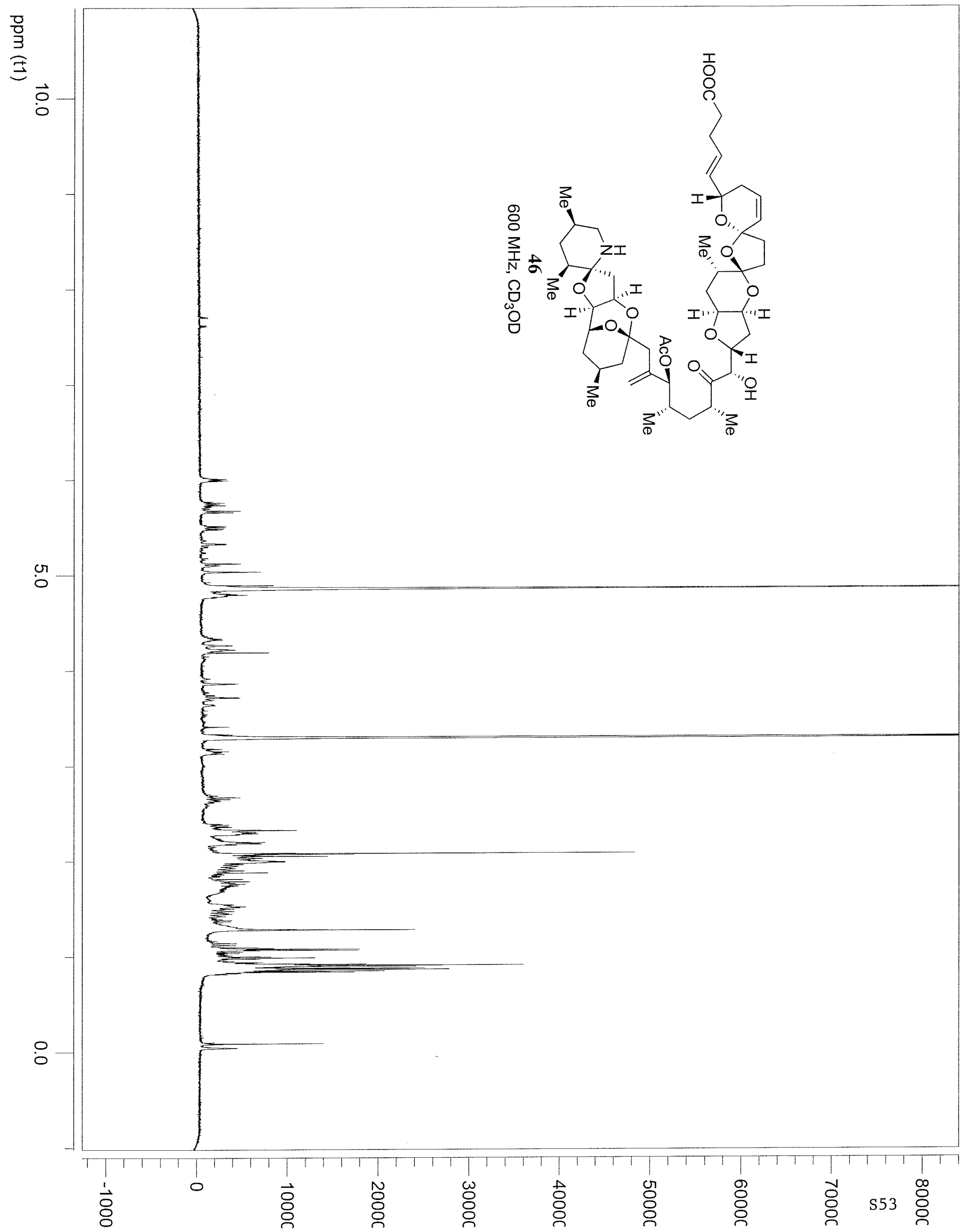




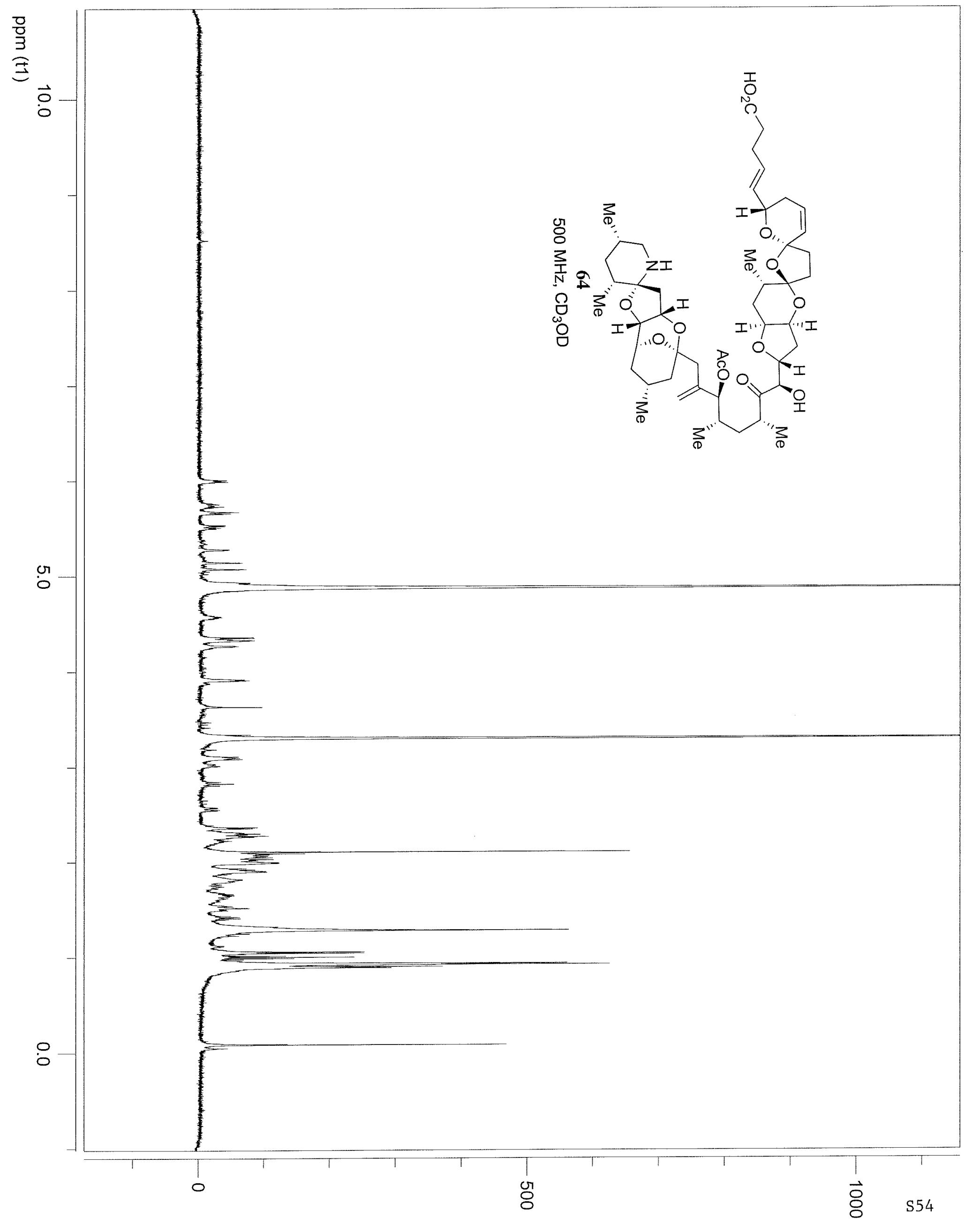




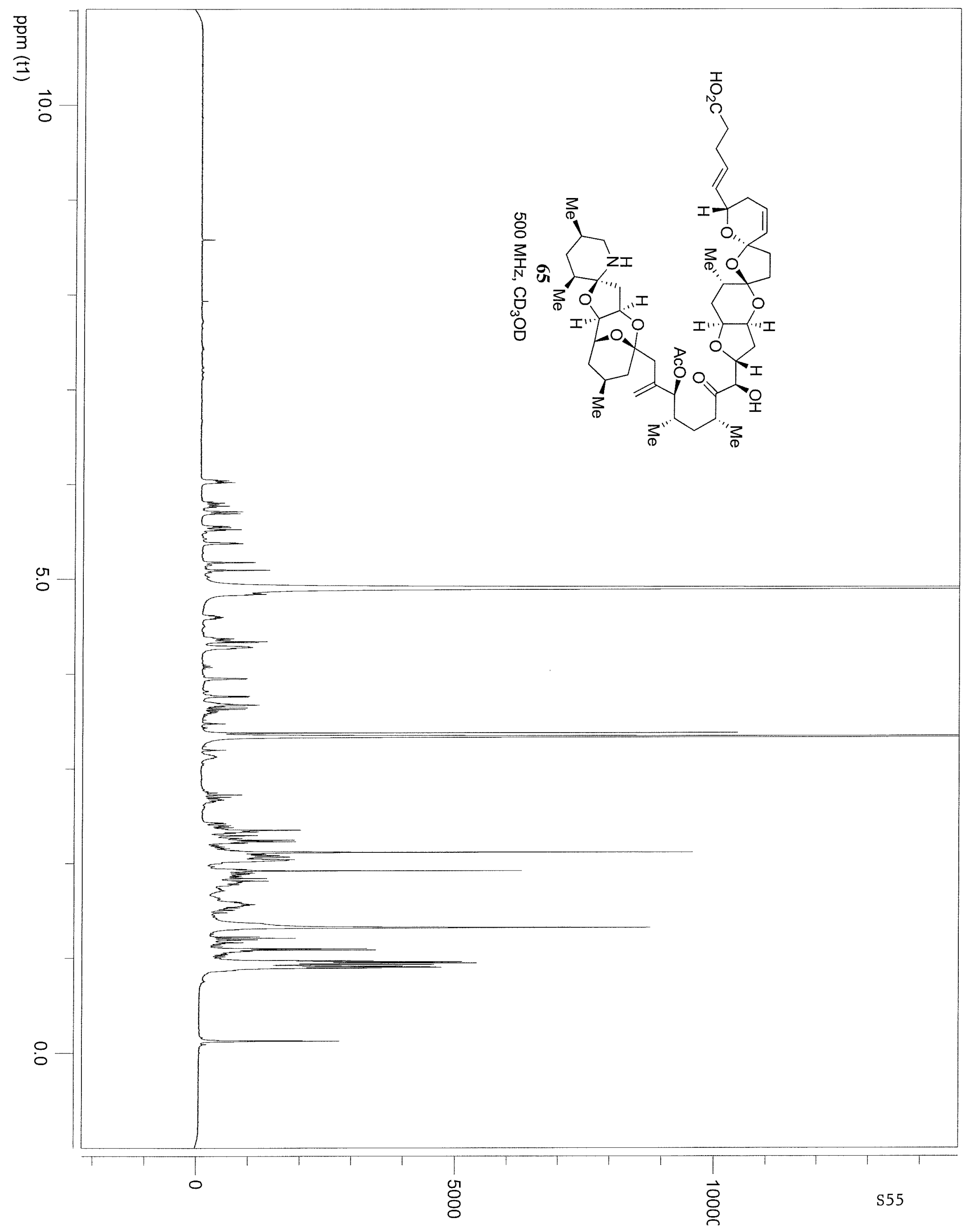




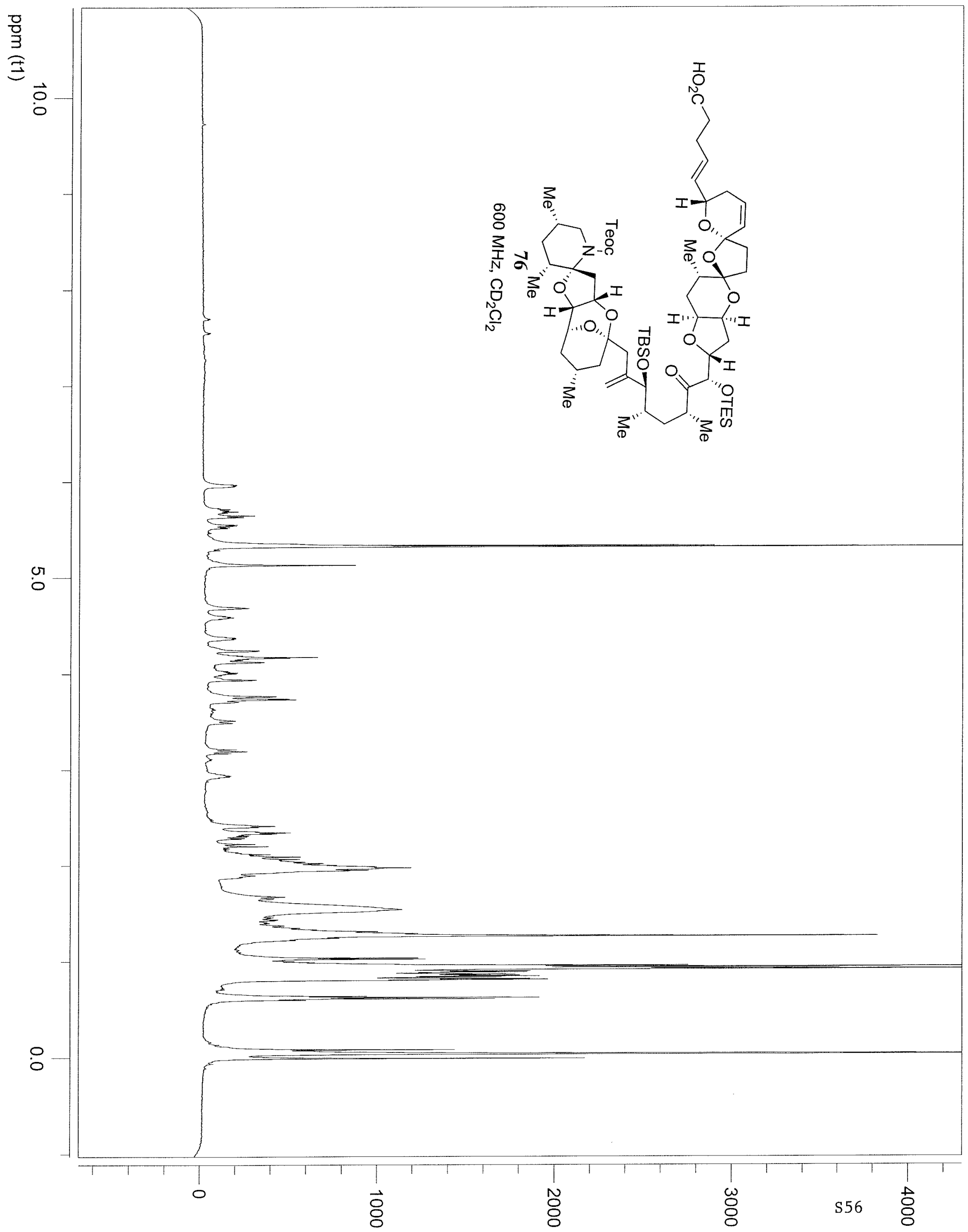

